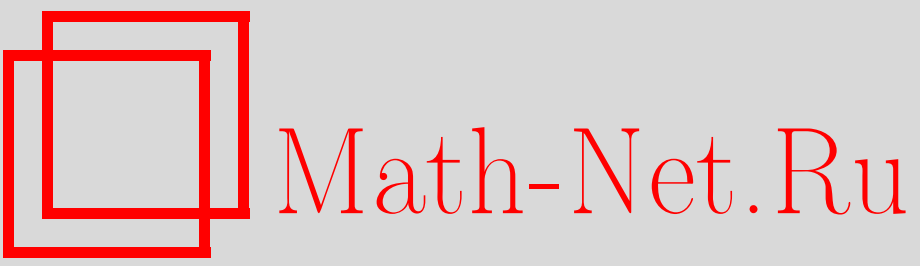

В. А. Исковских, В. В. Шокуров, Бирациональные модели и перестройки, УМН, 2005, том 60, выпуск 1, 29-98

DOI: https://doi.org/10.4213/rm1388

Использование Общероссийского математического портала Math-Net.Ru подразумевает, что вы прочитали и согласны с пользовательским соглашением

http://www.mathnet.ru/rus/agreement

Параметры загрузки:

IP : 54.210 .77 .194

26 апреля 2023 г., 13:35:13 


\title{
БИРАЦИОНАЛЬНЫЕ МОДЕЛИ И ПЕРЕСТРОЙКИ
}

\author{
В. А. Исковских, В. В. Шокуров
}

\begin{abstract}
В обзоре излагается содержание двух глав из теории логминимальных моделей, а именно, глава о различных понятиях модели в этой теории и глава о бирациональных перестройках - логфлипах, в основном в размерности 3. Изложение основано на идеях и резултатах второго автора: его работы о трехмерных логперестройках (а также труды семинара Университета шт. Юта на эту тему) для первой главы и работы о допредельных перестройках (см. также обзоры Корти и первого автора по этой теме) - для второй, где приводится полное доказательство существования логфлипа в размерности 3, являющееся на сегодняшний день самым простьм и доступным для широкого круга математиков.

Библиографиия: 41 название.
\end{abstract}

\section{СОДЕРЖАНИЕ}

Введение

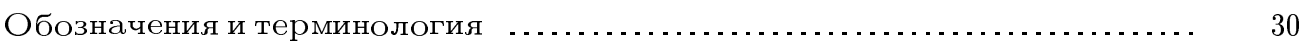

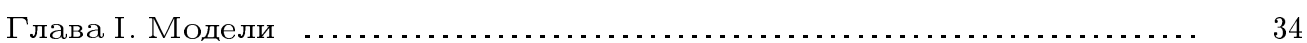

$\S 1$. Основные понятия логПММ …............................. 34

$\S 2$. Канонические и логканонические модели . . . . . . . . . . . . . . . . . . . 43

$\S 3$. Модификации логминимальных моделей: фрлопы и география логмо-

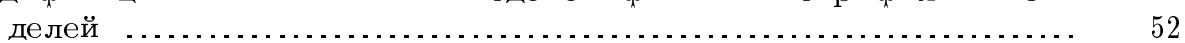

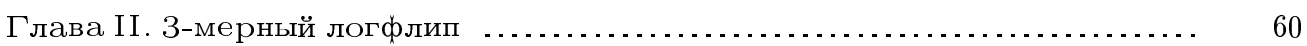

$\S 4$. Редукция к допредельным флипам и специальный обрыв $\ldots . . \ldots \ldots . . .60$

$\S 5$. Редукция 3-мерного допредельного фолипа в размерность 2 ........ 68

$\S 6$. Конечная порожденность FGА-алгебр в размерности $2 \ldots \ldots . \ldots . . .80$

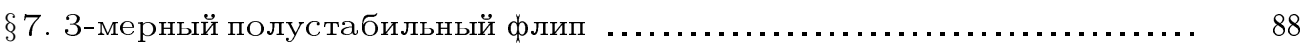

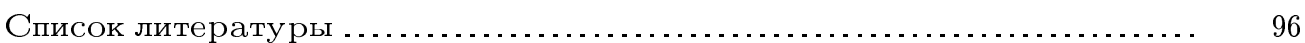

Работа первого автора вьполнена при финансовой поддержке Российского фонда фундаментальных исследований (проект 05-01-00343), а также гранта НШ-489.2003.1.

Работа второго автора вьполнена при финансовой поддержке NSF (гранты DMS-0100991 и DMS-0400832). 
Der Vogelsprecher bin ich ja, Stets lustig heißa hopsassa! Ich Vogelsprecher bin bekannt bei Alt und Jung im ganzen Land. Weiß mit dem Locken umzugehn und mich aufs Pfeifen zu verstehn!

Papageno's aria, Act I, Scene I, "Die Zauberflöte", W. A. Mozart

\section{Введение}

В этом обзоре мы излагаем (по возможности, в наиболее доступной форме для многих математиков) две важные главы из Программы лог-Минимальных Моделей (логПММ): о различных понятиях модели в этой теории и их свойствах (глава I), а также о бирациональных перестройках - логфлипах и логфлопах, в основном в размерности 3 (глава II). Все главные идеи и большинство результатов здесь принадлежат второму из авторов. Для первого автора - это один из курсов лекций, которьй он читал на механико-математическом факультете МГУ в 2003-2004 учебном году.

В главе II приводится полное доказательство существования логфлипов в размерности 3 , основанное на новом индуктивном подходе к этой проблеме второго из авторов (см. [37]). Авторам представляется, что это самое простое и короткое доказательство по сравнению с предыдушими [26], [34], [21]. В последнем параграфе обсуждается 3-мерный полустабильньй флип.

Основная цель (лог) ПММ - приложение этой программы в геометрии, что выходит за рамки нашего изложения. Типичные примеры такого приложения читатель найдет в [38] и других статьях данного цикла.

\section{Обозначения и терминология}

0.1. Как обычно, $\mathbb{N}, \mathbb{Z}, \mathbb{Q}, \mathbb{R}$ и $\mathbb{C}$ обозначают натуральные, целые, рациональные, вешественные и комплексные числа с соответствуюшими структурами. Основное поле $k$ предполагается, если не оговорено противное, алгебраически замкнутым характеристики нуль (часто - это просто поле комплексных чисел $\mathbb{C}$ ). Аналитичность понимается в комплексном смысле.

0.2. Морфизм (т.е. всюду определенное отображение алгебраических многообразий, пространств, схем и т. п.) обозначается $f: X \rightarrow Y$, рациональное (и мероморфное) отображение $-f: X \rightarrow Y$. Модификация - это бирациональное (бимероморфное) преобразование полных многообразий (компактных в случае комплексно аналитических пространств). Соответственно модификация собственного морфизма $f: X \rightarrow Z-$ это коммутативная диаграмма

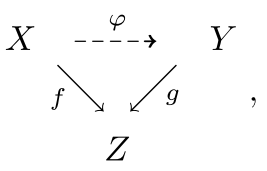


где $g: Y \rightarrow Z$ - собственньй морфизм, а $\varphi$ - бирациональное (бимероморфное) отображение.

Собственный морфизм $f: X \rightarrow Y$ называют стягиванием, если $f_{*} \mathscr{O}_{X}=\mathscr{O}_{Y}$ (т.е. в характеристике нуль и для нормальных многообразий $X, Y$ все слои $f$ связны). Если, кроме того, морфизм $f$ бирационален (бимероморфен), то он назьвается сдутием. Раздутие - это другое название для сдутия, когда оно строится по $Y$. Соответственно нормальное раздутие - это раздутие с нормальным многообразием $X$. Сдутие (раздутие) назьвается мальм. если его исключительное множество $\operatorname{Exc} f$ имеет коразмерность $\geqslant 2$.

0.3. Свободная абелева группа, порождаемая неприводимыми подмногообразиями коразмерности 1 на нормальном многообразии $X$, назьвается группой дивизоров Вейля $\operatorname{Div} X$, а данные образу ющие называются простьми дивизорами. Ее подгруппа дивизоров Картье состоит из всех локально главных дивизоров. $\mathbb{Q}-($ соответственно $\mathbb{R}-)$ дивизоры - это элементы $\operatorname{Div} X \otimes \mathbb{Q}$ (соответственно $\operatorname{Div} X \otimes \mathbb{R})$. $\mathbb{Q}$ - (соответственно $\mathbb{R}$-) дивизор $D=\sum d_{i} D_{i}$, где $D_{i}$ - простые дивизоры, $d_{i} \in \mathbb{Q}$ (соответственно $\left.d_{i} \in \mathbb{R}\right)$, назьвается $\mathbb{Q}$-Картье (соответственно $\mathbb{R}$-Картье), если $D=\sum r_{i} C_{i}$, где $C_{i}-$ дивизоры Картье и все кратности $r_{i} \in \mathbb{Q}\left(\right.$ соответственно $\left.r_{i} \in \mathbb{R}\right)$. $\mathbb{Q}$-дивизор $D$ является $\mathbb{Q}$-Картье, если и только если $m D$ - дивизор Картье для некоторого $m \neq 0 \in \mathbb{Z}$. $\mathbb{Q}$-дивизор является $\mathbb{R}$-Картье, если и только если он $\mathbb{Q}$-Картье.

Линейная эквивалентность дивизоров обозначается через $\sim$. $\mathbb{Q}$-линейная (соответственно $\mathbb{R}$-линейная) - через $\sim_{\mathbb{Q}}\left(\right.$ соответственно $\left.\sim_{\mathbb{R}}\right)$. Напомним, что $\mathbb{Q}$-дивизоры $D_{1}, D_{2} \in \operatorname{Div} X \otimes \mathbb{Q}$ (или даже $\mathbb{R}$-дивизоры $\left.D_{1}, D_{2} \in \operatorname{Div} X \otimes \mathbb{R}\right)$ назьваются $\mathbb{Q}$-линейно эквивалентными, если их разность $D_{1}-D_{2}$ является $\mathbb{Q}$-главным дивизором, т.е. $D_{1}-D_{2}=\sum d_{i}\left(f_{i}\right)$, где $d_{i} \in \mathbb{Q}$ и $f_{i} \neq 0 \in k(X)$ - рациональные (в аналитической ситуации мероморфные) функции на $X, \mathrm{a}\left(f_{i}\right)$ - их дивизор (нулей и полюсов). Аналогично для $\mathbb{R}$-дивизоров. Отметим, что если $D_{1} \mathbb{Q}-$ Картье и $D_{1} \sim_{\mathbb{Q}} D_{2}$, то и $D_{2}$ $\mathbb{Q}$-Картье; при этом эквивалентность $D_{1} \sim_{\mathbb{Q}} D_{2}$ равносильна линейной $m D_{1} \sim m D_{2}$ для некоторого $m \neq 0 \in \mathbb{Z}$.

Обозначим $\equiv$ численную эквивалентность для $\mathbb{Q}$ - и $\mathbb{R}$-дивизоров (она определена не только когда они $\mathbb{Q}$ - или $\mathbb{R}$-Картье): на полном многообразии $X$

$$
D_{1} \equiv D_{2} \Longleftrightarrow D_{1}-D_{2} \equiv 0 \Longleftrightarrow\left(D_{1}-D_{2}\right) C=0 \text { для всех кривых } C \subset X \text {; }
$$

при этом разность $D_{1}-D_{2}$ предполагается $\mathbb{Q}$ - или $\mathbb{R}$-Картье соответственно. Дивизор $D \in \operatorname{Div} X \otimes \mathbb{R}$ назьвается численно әффективныл $($ nef = numerically eventually free по английски), если он $\mathbb{R}$-Картье и $D C \geqslant 0$ для любой кривой $C \subset X$. В относительной ситуащии $f: X \rightarrow Z$ кривые $C \subset X$ берутся над $Z$, т.е. $f(C)=$ pt. $\in Z$. Тогда говорят, что $D$ численно эффективен над $Z$ или численно $f$-эффективен.

$\mathbb{R}$-дивизор $D$ назьвается облемнылм, если $h^{0}(X, m D)>$ const $m^{\operatorname{dim} X}$ для всех $m \gg 0$. Для численно эффективного $D$ это эквивалентно тому, что $D^{\operatorname{dim} X}>0$. Аналогично определена относительная объемность.

0.4. Канонический дивизор $K=K_{X}=(\omega)$ - это дивизор Вейля (нулей и полюсов) любой рациональной (мероморфной) дифференциальной формы $\omega \neq 0$ старшей степени на $X$. Многообразие $X$ называется горенштейновым, если $K$ - дивизор Картье, $\mathbb{Q}$-горенштейновьм, если $K \mathbb{Q}$-Картье, т.е. $m K$ Картье для некоторого $m \in \mathbb{Z} \backslash\{0\}$. Для точки $x \in X$ наименьшее натуральное $m_{x}>0$ такое, что $m_{x} K$ 
локально главный в $x$, называется (локальнылм) индексом (горенштейновости) точки $x$, а наименьшее общее кратное множества $\left\{m_{x} \mid x \in X\right\}$ всех индексов назьвается (глобальным и) индексом (горенштейновости) $X$.

Аналогично определяется индекс логпары $(X, B)$ как индекс присоединенного дивизора $K+B$. В качестве $B$ тогда берется $\mathbb{Q}$-граница (или $\mathbb{Q}$-дивизор). В общем случае $\mathbb{R}$-гранича, или просто граница, есть $\mathbb{R}$-дивизор $B=\sum b_{i} D_{i}$, где суммирование берется по простым дивизорам $D_{i}$ и все кратности $b_{i} \in \mathbb{R}, 0 \leqslant b_{i} \leqslant 1$. Для $\mathbb{Q}$-гранииьи все $b_{i} \in \mathbb{Q}$.

Многообразие $X$ назьвается $\mathbb{Q}$-факториальным, если всякий дивизор Вейля на $X$ является $\mathbb{Q}$-Картье.

Для любого $\mathbb{R}$-дивизора $D=\sum d_{i} D_{i}$ следующие обозначения обшеприняты:

$\lceil D\rceil:=\sum\left\lceil d_{i}\right\rceil D_{i}-$ верхняя целая часть;

$\lfloor D\rfloor:=\sum\left\lfloor d_{i}\right\rfloor D_{i}-$ нижняя (обычная) целая часть;

для любого вешественного числа $d \in \mathbb{R}$ символ $\lceil d\rceil$ обозначает наименьшее целое число $\geqslant d$, a $\lfloor d\rfloor$ - наибольшее целое $\leqslant d$.

Для $\mathbb{R}$-дивизора $D$ положим $H^{i}(X, D)=H^{i}\left(X, \mathscr{O}_{X}(D)\right), h^{i}(X, D)=h^{i}\left(X, \mathscr{O}_{X}(D)\right)$, где $\mathscr{O}_{X}(D)=\mathscr{O}_{X}(\lfloor D\rfloor)$ - дивизориальньй пучок, ассоциированный с $D$.

Алгебраическая замкнутость основного поля не нужна, когда простой дивизор Вейля понимается алгебраически, а не геометрически. То же самое относится к циклам.

0.5. Напомним теперь важнейшее понятие теории - конус Клеймана-Мори - замыкание конуса эффективных 1-циклов. 1-цикл на $X$ - это линейная комбинация $z=$ $\sum n_{i} C_{i} \in \mathrm{Z}_{1} X$, где суммирование берется по простым 1-циклам $C_{i} \subset X$ - кривым и все кратности $n_{i} \in \mathbb{Z}$. Если все $n_{i} \geqslant 0$, цикл называют әффективным. Следуюшие понятия и обозначения общеприняты: для полного алгебраического многообразия $X$

$\mathrm{N}(X):=\mathrm{N}_{1} X=\mathrm{Z}_{1} X \otimes \mathbb{R} /(\bmod \equiv) ;$ численная эквивалентность $\equiv 1$-циклов двойственна численной эквивалентности дивизоров:

$z_{1} \equiv z_{2} \Longleftrightarrow z_{1}-z_{2} \equiv 0 \Longleftrightarrow D\left(z_{1}-z_{2}\right)=0$ для всех дивизоров Картье $D$ на $X$;

$\rho(X)=\operatorname{dim}_{\mathbb{R}} \mathrm{N}(X)$ - число Пикара (совпадает с рангом групшы Нерона-Севери); $\mathrm{NE}(X)$ - конус эффективных 1-циклов; конус $\mathrm{NE}(X)$ порожден в векторном пространстве $\mathrm{N}(X) \cong \mathbb{R}^{\rho(X)}$ классами эффективных 1-циклов, т.е. векторы конуса суть классы по модулю численной эквивалентности эффективных вещественных 1-циклов $z=\sum r_{i} C_{i}, r_{i} \in \mathbb{R}, r_{i} \geqslant 0$;

$\overline{\mathrm{NE}}(X)$ - конус Клеймана-Мори, где замыкание - берется в обычной вешественной топологии.

Пространство $\mathrm{N}(X)$ также обозначают как $\mathrm{N}_{1} X$ в противоположность двойственному пространству $\mathrm{N}^{1} X$, являюшемуся факторпространством $\mathbb{R}$-Картье дивизоров на $X$ по модулю численной эквивалентности $\equiv$. Таким образом, всякий $\mathbb{R}-$ Картье дивизор $D$ можно рассматривать как $\mathbb{R}$-значную линейную функцию на $\mathrm{N}(X)$, индуцированную пересечением $D z=\sum r_{i} D C_{i}, r_{i} \in \mathbb{R}$.

По критерию обильности Клеймана

$$
\mathbb{Q} \text {-Картье } D \text { обилен } \Longleftrightarrow D z>0 \text { на } \overline{\mathrm{NE}}(X) \backslash\{0\} \text {; }
$$


если $D-\mathbb{R}$-Картье дивизор, удовлетворяюший второму условию, и $X$ проективно, то $D$ называется численно обильным. Последнее эквивалентно существованию полулинейного разложения: $D=\sum h_{i} H_{i}, h_{i} \in \mathbb{R}, h_{i} \geqslant 0$, где все $H_{i}$ обильны.

При определении соответствующих относительных понятий для собственного морфизма $f: X \rightarrow Z$ 1-циклы $z$ предполагаются лежашими над $Z$, т.е. $f_{*} z=0$. Так, например, $\mathbb{R}$-Картье дивизор $D$ (относительно) численно обилен над $Z$ или $f$-обилен, если $D z>0$ на $\overline{\mathrm{NE}}(X / Z) \backslash\{0\}$.

Стягивание (сдутие) $f: X \rightarrow Z$ назьвается экстремальным, если относительное число Пикара $\rho(X / Z)=\rho(X)-\rho(Z)=1$.

0.6. Экстремальные лучи. Пусть $(X, D)$ - полная логпара, т.е. $X$ - полное нормальное многообразие и $D-\mathbb{R}$-дивизор такой, что присоединенньй дивизор $K+D$ $\mathbb{R}$-Картье. Положим

$\overline{\mathrm{NE}}_{\geqslant 0}(X, D):=\{z \in \overline{\mathrm{NE}}(X) \mid(K+D) z \geqslant 0\}-(K+D)$-положительная часть конуса Клеймана-Мори;

$\overline{\mathrm{NE}}_{<0}(X, D):=\{z \in \overline{\mathrm{NE}}(X) \mid(K+D) z<0$ или $z=0\}-(K+D)$-отрицательная часть конуса Клеймана-Мори.

Для каждого $\mathbb{R}$-Картье дивизора $H$ определена двойственная гиперплоскость $H^{\perp}:=$ $\{z \in \mathrm{N}(X) \mid H z=0\}$ в $\mathrm{N}(X)$. Дивизор $H$ численно эффективен, если и только если $H^{\perp}$ - опорная гиперплоскость конуса $\overline{\mathrm{NE}}(X)$ и последний конус лежит целиком в положительном полупространстве $\{z \in \mathrm{N}(X) \mid H z \geqslant 0\}$. При этом подмножество $F=\overline{\mathrm{NE}}(X) \cap H^{\perp} \subset \overline{\mathrm{NE}}(X)$ является гранью конуса. 1-мерная грань $R \subset \overline{\mathrm{NE}}(X)$ называется экстремальным лучом. Экстремальный луч задан относительно $K+D$, если $R \subset \overline{\mathrm{NE}}_{<0}(X, D)$ или, как часто пишут, $(K+D) R<0$. В общем случае не всякая грань (луч) порождена кривьми.

По теореме о конусе [1; Theorem 2], если $(X, B)$ - логканоническая логпара (например, $\mathbb{Q}$-факториальная и логтерминальная) с границей $B$ и $X$ проективно, то

$$
\overline{\mathrm{NE}}(X)=\overline{\mathrm{NE}}_{\geqslant 0}(X, B)+\sum R_{l},
$$

где $R_{l}$ пробегает (не более чем счетное) множество всех экстремальных лучей относительно $K+B$. Более того, конус $\overline{\mathrm{NE}}(X)$ локально кополиэдрален в отрицательной части $\overline{\mathrm{NE}}_{<0}(X, B)$ и $\overline{\mathrm{NE}}_{<0}(X, B)=\mathrm{NE}_{<0}(X, B):=\{z \in \mathrm{NE}(X) \mid$ $(K+B) z<0$ или $z=0\}$, т.е. лучи $R_{l}$ дискретны и конус эффективных 1-циклов замкнут в $(K+B)$-отрицательной части. При этом каждый луч $R_{l}$ имеет вид $R_{l}=\mathbb{R}_{+}\left[C_{l}\right]$, где $\left[C_{l}\right] \in \overline{\mathrm{NE}}(X)-$ класс некоторой рациональной кривой $C_{l} \subset X$ и $\mathbb{R}_{+}=\{r \in \mathbb{R} \mid r \geqslant 0\}$.

В относительной ситуации полная логпара $(X, D)$ заменяется собственной относительной парой $f:(X, D) \rightarrow Z$, т.е. морфизм $f: X \rightarrow Z$ предполагается собственным; проективность - относительной проективностью.

0.7. Циклические и гиперповерхностные факторособенности. Пусть $\mathbb{Z}_{r} \cong$ $\mu_{r} \subset k$ - циклическая группа порядка $r \geqslant 1$, рассматриваемая как группа корней из 1 степени $r$. Тогда она естественно действует на пространстве $k^{n}$ с целыми весами $\left(a_{1}, \ldots, a_{n}\right), a_{i} \in \mathbb{Z}:$

$$
\left(x_{1}, \ldots, x_{n}\right) \mapsto\left(\zeta^{a_{1}} x_{1}, \ldots, \zeta^{a_{n}} x_{n}\right),
$$


где $\zeta \in \mu_{r}$. Обычно предполагается взаимная простота $\left(a_{1}, \ldots, a_{n}\right)=1$. Тогда действие свободно в коразмерности 1. Фактормногообразие $X=k^{n} / \mu_{r}=k^{n} / \mathbb{Z}_{r}\left(a_{1}, \ldots, a_{n}\right)$ имеет циклические факторособенности и обозначается через $\frac{1}{r}\left(a_{1}, \ldots, a_{n}\right)$, как и тип точки 0 , отвечающей $(0, \ldots, 0) \in k^{n}$, с точностью до локального (аналитического или формального) изоморфизма.

Аналогично, если $0 \in Y=(F=0) \subset k^{n+1}-$ нормальная гиперповерхностная особенность с действием групшы $\mu_{r}$ и $0 \in X=Y / \mu_{r}$ - соответствующая особенность фактора. Тогда действие $\mu_{r}$ может быть продолжено на $k^{n+1}$ (как действие на кокасательном пространстве в 0 в подходяших координатах) как выше с весами $\left(a_{0}, \ldots, a_{n}\right)$. Так как гиперповерхность $Y$ инвариантна относительно этого действия, то $F=\zeta^{e} F$ - (полуинвариантная) собственная функция действия. Символ $\frac{1}{r}\left(a_{0}, \ldots, a_{n} ; e\right)$ или, точнее, $(F=0) \subset \frac{1}{r}\left(a_{0}, \ldots, a_{n}\right)$ обозначает тип соответствующей гиперповерхностной факторособенности $0 \in X$.

0.8. Взвешенное раздутие. Рассмотрим циклическую факторособенность $0 \in$ $X:=k^{n} / \mathbb{Z}_{r}\left(a_{1}, \ldots, a_{n}\right), r \geqslant 1,\left(a_{1}, \ldots, a_{n}\right)=1, x_{1}, \ldots, x_{n}$-координаты, собственные относительно действия $\left(X=k^{n}\right.$ для $\left.r=1\right)$. Под взвешенньм раздутием $X$ с весами $\frac{1}{r}\left(a_{1}, \ldots, a_{n}\right)$ понимается раздутие $\sigma: Y \rightarrow X$ такое, что $Y$ покрьвается аффинными картами $U_{1}, \ldots, U_{n}$ вида

$$
U_{i}=k^{n} / \mathbb{Z}_{a_{i}}\left(a_{1}, \ldots, \stackrel{i}{r}, \ldots, a_{n}\right)
$$

Координаты $x_{j}$ на $X$ и $y_{j}$ на $U_{i}$ связаны формулами $y_{j}=x_{j} / x_{i}^{a_{j} / a_{i}}, y_{i}=x_{i}^{r / a_{i}}$. Исключительное подмножество $E$ для $\sigma$ является $\mathbb{Q}$-Картье дивизором Вейля, $E \cap U_{i}=$ $\left(y_{i}=0\right) \subset k^{n} / \mathbb{Z}_{a_{i}}\left(a_{1}, \ldots, r, \ldots, a_{n}\right), E=\operatorname{Proj}\left(a_{1}, \ldots, a_{n}\right)$ - взвешенное проективное пространство размерности $n-1$. (См. [8; гл. II, 3.6] с точностью до обозначения весов.)

ЗАМЕЧАНИЕ 0.9. Большинство результатов обзора легко переносятся и доказываются в аналитической и формальной категориях. Например, в аналитической категории относительную логПММ обычно рассматривают над окрестностью компактного подмножества базы [11; Section 1], [27].

\section{Глава I. Модели}

В этой главе мы напомним основные понятия, установившиеся в Программе логМинимальных Моделей (логПММ), и воспроизведем некоторые результаты, используемые в дальнейшем. Более подробно остановимся на понятии модели в логПММ и дадим интерпретацию известного неравенства Нётера-Фано в этих терминах.

\section{$\S$ 1. Основные понятия логПММ}

1.1. Пусть $(X, D)$ - пара, состоящая из полного нормального алгебраического многообразия $X$ (обычно над некоторой базой $Z$, которую мы пока опускаем в обозначениях ) и не обязательно эффективного $\mathbb{R}$-Картье дивизора $D$. Под Программой $D$ - $М и н и-$ мальных Моделей ( $D$-ПММ) понимается последовательность модификаций

$$
\left(X_{0}, D_{0}\right)_{\stackrel{g_{0}}{\rightarrow}}^{\rightarrow}\left(X_{1}, D_{1}\right)_{\stackrel{-}{g_{1}} \rightarrow}^{\rightarrow}\left(X_{2}, D_{2}\right)^{\stackrel{g_{2}}{\rightarrow}} \cdots,
$$


которая строится следующим образом.

а) $\left(X_{0}, D_{0}\right)$ - исходная пара, например, равная $(X, D)$, когда последняя удовлетворяет определенным свойствам, сохраняемьм преобразованиями в с) и d) ниже. Обычно требуют проективность $X_{0}$ и некоторые условия неособости пары $\left(X_{0}, D_{0}\right)$. Если $X$ не проективно или пара $(X, D)$ особа, то берется ее подходящая модель $\left(X_{0}, D_{0}\right)$ (в бирациональном смысле), при этом возможны различные подходы к определению $D_{0}$.

b) Предположим, что пара $\left(X_{i}, D_{i}\right)$ уже построена. Если $\mathbb{R}-$ Картье дивизор $D_{i}$ численно эффективен, то алгоритм закончен - это по определению $D$-минимальная модель для $(X, D)$.

c) Если $D_{i}$ не численно эффективен, тогда нужно найти стягивание $\varphi_{i}: X_{i} \rightarrow Y_{i}$ такое, что дивизор $-D_{i}$ относительно численно $\varphi_{i}$-обилен и $\rho\left(X_{i} / Y_{i}\right)=1$, где $\rho(\cdot / \cdot)$ обозначает, как обычно, (относительное) число Пикара. Такое стягивание называют $D_{i}$-стягиванием (или $D$-стягиванием). В случае, когда $\varphi_{i}$ - не сдутие, т.е. морфизм расслоенного типа, алгоритм опять считается законченным. Если $\varphi_{i}-$ сдутие и $\varphi\left(D_{i}\right)-\mathbb{R}$-Картье дивизор (что обычно случается, когда исключительное множество морфизма $\varphi_{i}$ неприводимо и дивизориально), то положим $g_{i}:=\varphi_{i}$ и $\left(X_{i+1}, D_{i+1}\right):=$ $\left(Y_{i}, \varphi_{i}\left(D_{i}\right)\right)$. (Программа не работает, если $\varphi_{i}$ не существует!)

d) Если $\varphi_{i}\left(D_{i}\right)$ не является $\mathbb{R}$-Картье дивизором, то, чтобы продолжить процесс, надо найти коммутативную диаграмму вида

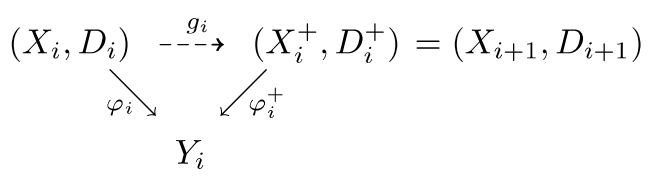

со следуюшими свойствами:

(i) $\varphi_{i}^{+}-$малое сдутие, т.е. его исключительное множество $\operatorname{Exc} \varphi_{i}^{+}$имеет коразмерность $\geqslant 2$

(ii) $D_{i+1}=D_{i}^{+}=g_{i *} D_{i}$;

(iii) $D_{i}^{+}$численно $\varphi_{i}^{+}$-обилен.

Диаграмма (1.1) с этими свойствами называется $D_{i}$-флипом (или $D$-флипом или $n e-$ рестройкою) сдутия $\varphi_{i}$. Если $D_{i}$-флип существует, то алгоритм построения $D$-минимальной модели может быть продолжен. Кроме вопросов существования возникает естественный вопрос обрыв $а$, т.е. конечности алгоритма. Если это так, то в конце алгоритма получаем результирующую пару: либо $D$-минимальную модель как в b), либо расслоенньй морфизм как в с).

Обрьв дивизориальных сдутий в с) следует из уменьшения числа Пикара $\rho\left(X_{i}\right)$ для таких сдутий. Поэтому требуется установить обрьв лишш флипов, что и подразумевается всюду в дальнейшем.

Проблема существования $D$-минимальных моделей зависит прежде всего от характера особенностей исходной пары $\left(X_{0}, D_{0}\right)=(X, D)$. Например, если $D=K=K_{X}-$ канонический дивизор и $X-$ проективное $\mathbb{Q}$-факториальное многообразие с (не более чем) терминальными особенностями - это обычная Программа Минимальных Моделей (ПММ) алгебраических многообразий или теория Мори, а если $D=K+B$, где 
$B=\sum b_{i} D_{i}-$ границ $a$, т.е. $0 \leqslant b_{i} \leqslant 1$, суммирование ведется по простым дивизорам $D_{i}$, и пара $(X, B)$ имеет $\mathbb{Q}$-факториальные логтерминальные (или даже логканонические) особенности, то это логПММ (см., например, [22], [17], [34], [36]). Если $X$ не проективно или пара $(X, B)$ имеет худшие особенности, то в качестве исходной модели берется ее проективное разрешение $\left(X_{0}, B_{0}\right)$.

$\mathrm{B}$ связи с этим напомним основные определения. Ниже $D$ обозначает нечто другое, а именно - часть присоединенного дивизора.

ОПРЕДЕЛЕНИЕ 1.2. Пусть $X$ - нормальное многообразие и $D=\sum d_{i} D_{i}, d_{i} \in \mathbb{R},-$ некоторьй $\mathbb{R}$-дивизор (не обязательно эффективньй) такой, что присоединенный дивизор $K+D$ является $\mathbb{R}$-Картье, где $K=K_{X}$ - канонический дивизор многообразия $X$, а $D_{i}$ - простые дивизоры Вейля. Пусть $f: Y \rightarrow X$ - нормальное раздутие многообразия $X$. Тогда при подходяшем выборе канонического дивизора $K_{Y}$ можно записать

$$
K_{Y}=f^{*}(K+D)+\sum a(E, X, D) E,
$$

где $E \subset Y$ - простые дивизоры Вейля, $a(E, X, D) \in \mathbb{R}$ - вешественные числа. Если $E=D_{i}-$ не исключительньй дивизор на $X$, то как видно из предыдушей формулы $a\left(D_{i}, X, D\right)=-d_{i}$. Числа $a(E, X, D)$ называются дискрепантностями пары $(X, D)$ в $E$, они зависят только от дивизориального нормирования поля $k(X)$ или простого b-дивизора $\underline{E}=E$ (см. определение 5.5 ниже), определяемого $E$ и отождествляемого с $E$, но не от $Y$ и выбора $K, K_{Y}$. Образ $f(E)$ называется центром $E$ на $X$ (или определяемого им нормирования, b-дивизора) и обозначается через $\mathrm{c}_{X} E . E-$ исключительный на $X$, если $\mathrm{c}_{X} E$ имеет коразмерность $\geqslant 2$. Простые b-дивизоры многообразия $X$ суть простые b-дивизоры или дивизориальные нормирования поля $k(X)$ с непустыми центрами на $X$.

Выбор дивизора $K_{Y}$ определяется выбором $K$ : если $K=(\omega)$ - дивизор нулей и полюсов рациональной дифференциальной формы $\omega \neq 0$ на $X$, то $K_{Y}=\left(f^{*} \omega\right)$ - дивизор формы $f^{*} \omega$. При произвольном выборе $K_{Y}$ равенство $=$ надо заменить линейной эквивалентностью .

Определим дискрепантность $\operatorname{dis}(X, D)$ и тотальную дискрепантность $\operatorname{tdis}(X, D)$ пары $(X, D)$ следуюшим образом:

$$
\begin{gathered}
\operatorname{dis}(X, D):=\inf _{E}\{a(E, X, D) \mid E-\text { все исключительнье на } X \\
\text { простые b-дивизоры многообразия } X\}, \\
\operatorname{tdis}(X, D):=\inf _{E}\{a(E, X, D) \mid E-\text { все простые b-дивизоры } \\
\text { многообразия } X\} .
\end{gathered}
$$

ОПРЕДЕЛЕНИЕ 1.3. Пусть $X$ - нормальное многообразие, $D=\sum d_{i} D_{i}-\mathbb{R}$-дивизор такие, что присоединенньй дивизор $K+D$ является $\mathbb{R}$-Картье. Говорят, что пара $(X, D)$ (или просто $K+D$ ) обладает следуюшими особенностями при выполнении со- 
ответствуюших неравенств:

$$
\left.\begin{array}{l}
\text { терминальными }(\mathrm{trm}) \\
\text { каноническими }(\mathrm{cn}) \\
\text { чисто логтерминальными (plt) } \\
\text { логтерминальными по Кавамате }(\mathrm{klt}) \\
\text { логканоническими (lc) }
\end{array}\right\} \Longleftrightarrow \operatorname{dis}(X, D)\left\{\begin{array}{l}
>0 ; \\
\geqslant 0 ; \\
>-1 ; \\
>-1 \text { и } d_{i}<1 \forall i ; \\
\geqslant-1 \text { и } d_{i} \leqslant 1 \forall i .
\end{array}\right.
$$

ЗАмЕчАнИя 1.4. (i) Кроме этих особенностей широко используются также следующие:

пара $(X, D)$ логтерминальна (lt), если существует ее логразрешение $f: Y \rightarrow X$ с $a(E, X, D)>-1$ для всех исключительных дивизоров $E$ морфизма $f$;

$(X, D)$ дивизориально логтерминальна (dlt), если существует логразрешение $f: Y \rightarrow X$ с дивизориальным исключительным множеством такое, что $a(E, X, D)>-1$ для всех исключительных дивизоров.

Эти определения используются, например, для работы с не $\mathbb{Q}$-факториальными многообразиями.

По определению

$$
\text { klt } \Rightarrow \text { plt } \Rightarrow \text { lt } \text { и dlt } \Rightarrow \text { lt. }
$$

Простые примеры показывают, что все классы особенностей различны.

Напомним, что логразрешением пары $(X, D)$ назьвается раздутие $Y \rightarrow X$ такое, что многообразие $Y$ неособо и простые компоненты собственного прообраза $D_{Y}$ дивизора $D$ вместе с исключительными дивизорами $E_{i}$ (логсобственный прообраз) неособы и имеют только нормальные пересечения (что обычно обозначается как $\operatorname{Supp}\left(D_{Y}\right)$ $\cup\left(\bigcup E_{i}\right)$ - дивизор с простыми нормальными пересечениями). Пара $\left(Y, D_{Y}+\sum E_{i}\right)$ называется логнеособой и если $D_{Y}-$ граница, то обычно годится в качестве исходной модели для логПММ.

(ii) В определении 1.2 inf является на самом деле min и $\geqslant-1$, или $=-\infty$. Первое - потому, что конечные dis и tdis вычислимы на дивизорах любого логразрешения (достаточно нетривиального в случае dis) и при дальнейших раздутиях не меняются.

(iii) В предыдущих определениях присоединенный $\mathbb{R}$-дивизор $K+D$ можно заменить $\mathbb{Q}$-дивизором $K+D^{\prime}$ без изменения рациональных чисел пересечения с кривыми и рациональных дискрепантностей (см. [34; $(1.3 .2)][21 ; 2.12])$.

(iv) Если $\operatorname{dim} X \geqslant 2$, то условие $\operatorname{tdis}(X, D) \geqslant-1$ равносильно $\operatorname{dis}(X, D) \geqslant-1$ и в определении lс можно опустить требование $d_{i} \leqslant 1 \forall i$.

ПРЕДЛОЖЕНИЕ $1.5[34 ; 1.3][21 ; 2.17])$. Пусть $X$ - нормальное многообразие и $D=\sum d_{i} D_{i}-\mathbb{R}-д$ ивизор. Тогда

(i) множество дивизоров $D$, для которых $K+D$ lc (численно әффективен, или численно обилен), является выпуклым подмножсеством в прямой сумме слагаемых $\mathbb{R} ;$ если $D$ - граница, то в прямой сумме единичных отрезков $[0,1]$;

(ii) множество дивизоров $D$ с носителями в фиксированных $D_{1}, \ldots, D_{s}$, для которых $K+D \mathrm{lc}$, является конечным выпукльм рациональным полиэдром в $\sum \mathbb{R} D_{i} ;$ если $D$ - граница, то компактным подмножеством в $\sum[0,1] D_{i}$; 
(iii) если $D^{\prime} \leqslant D$ и $K+D$ lc (соответственно lt) $u K+D^{\prime} \mathbb{R}-$ Kартье, то $K+D^{\prime}$ также lc (соответственно $\mathrm{lt}$ ), более того, $a(E, X, D) \leqslant a\left(E, X, D^{\prime}\right)$ для всех $E$;

(iv) nусть $K+D \mathrm{lt}$, тогда существует $\varepsilon>0$ такое, что $K+D^{\prime}$ lt для всех $\mathbb{R}$-Картье дивизоров $K+D^{\prime}$, где $D^{\prime}=\sum d_{i}^{\prime} D_{i}$ с $d_{i}^{\prime} \leqslant \min \left\{1, d_{i}+\varepsilon\right\} ;$ кроме того, если $K+D$ plt, то $K+D^{\prime}$ также plt;

(v) если $K+D$ plt $u K+D+D^{\prime}$ lc, то $K+D+t D^{\prime}$ plt

Теперь рассмотрим всевозможные понятия модели. Мы используем общепринятое понятие логпарьи (или логмногообразия). Логпара - это пара $(X, B)$, где $X$ - нормальное многообразие, а $B=\sum b_{i} D_{i}-\mathbb{R}$-гранича или просто граница (где $b_{i} \in \mathbb{R}$, $\left.0 \leqslant b_{i} \leqslant 1\right), D_{i}-$ простые дивизоры Вейля на $X$. Логканонический дивизор - это дивизор вида $K+B$, называемый также присоединенным дивизором. Отметим, что логПММ является $D$-ПММ, где в качестве $D$ берется именно присоединенный дивизор $K+B$.

ОПРЕДЕЛЕНИЕ $1.6[34 ; \S 1]$, [21; Section 2]). (i) Логпара $(X, B)$ с $\mathbb{R}$-границей $B$ называется логминимальной моделью, если дивизор $K+B$ численно эффективен (это предполагает, что он $\mathbb{R}$-Картье, а $X$ полно) и пара $(X, B)$ lt (или дивизор $K+B$ lt).

(ii) Логпара $(X, B)$ назьвается логканонической моделью, если дивизор $K+B$ численно обилен и пара $(X, B)$ lc. В случае, когда особенности cn, пара $(X, B)$ называется канонической моделью.

(iii) Логпара $(X, B)$ назьвается слабо логканонической моделью, если дивизор $K+B$ численно эффективен и $(X, B)$ lc. В случае, когда особенности trm, пара $(X, B)$ называется терминальной или минимальной. Слабо каноническая модель имеет сп особенности.

(iv) Пусть $(X, B)$ - слабо логканоническая модель и предположим, что дивизор $K+B$ полуобилен (гипотеза 1.16 ниже о полуобильности утверждает, что это предположение всегда вьполнено, а согласно [17; 6-1-13] для $\mathbb{Q}$-границы $B$ оно вытекает из гипотезы об избыточности [17; 6-1-14]). Стягивание $I(X, B): X \rightarrow U$, определяющее полуобильность $K+B$, назьвается расслоением Иитаки, а его образ $U$ - (логканонической) моделью Иитаки пары $(X, B)$; иногда он обозначается через $X_{\text {сап }}$. Размерность образа $\operatorname{dim} U$ совпадает с иисленной логкодаировой размерностью пары $(X, B)[36 ; 2.4 .4]$, обозначаемой через $\nu(X, B)$, и в случае $\mathbb{Q}$-границы $B$ является логкодаировой размерностью $\kappa(X, B)$ пары $(X, B)$ (по поводу обобщений см. замечание 1.7 (iii) ниже).

(v) Собственное стягивание $\varphi: X \rightarrow S$ нормальных многообразий назьвается $p a c-$ слоением Мори, если вьполнены следуюшие условия:

a) $\operatorname{dim} S<\operatorname{dim} X$;

b) $X$ имеет не более чем $\mathbb{Q}$-факториальные терминальные особенности;

c) $\rho(X / S):=\rho(X)-\rho(S)=1$, где $\rho(\cdot)$ - число Пикара;

d) $-K$ $\varphi$-обилен.

Условия а)-d) вместе означают, что $\varphi: X \rightarrow S$ является экстремальным $K$-стягиванием расслоенного типа - объектом категории Мори. При определении лограсслоения Мори пары $(X, B)$ надо в п. d) заменить $-K$ на численно $\varphi$-обильный $-(K+B)$, а в п. b) вместо терминальности предполагать логтерминальность $(X, B)$. 
Аналогично для лограсслоения Фано - lc вместо lt и не требуются условия а), $\mathbb{Q}$ факториальность в b) и с) (и даже возможно, что $\varphi$ - не стягивание, а лишь собственный морфизм).

Относительный вариант этих понятий определяется стандартным образом.

ЗАмЕчАния 1.7. (i) Логминимальная (относительная) модель назьвается также логтерминальной (относительной) моделью [34; с. 111]. Используется также понятие (относительной) строго логминимальной или (относительной) строго логтерминальной модели, когда многообразие $X$, кроме того, $\mathbb{Q}$-факториально и (относительно) проективно.

(ii) Конечно, если $B=0$, то основная гипотеза теории Мори утверждает, что либо существует проективная $\mathbb{Q}$-факториальная (терминальная) минимальная модель (см. определение 1.9 (ii)), либо исходное многообразие бирационально эквивалентно расслоению Мори (что доказано, как известно, в размерности $\leqslant 4$ [17], [37]). Аналогичные результаты в логтерминальной категории доказаны пока только в размерности $\leqslant 3$.

(iii) Возможны обобщения для многообразий с субграницами, т.е. с $b_{i} \leqslant 1$ и, возможно, отрицательными. Они встречаются во вспомогательных конструкциях, например, при логприсоединении $[34 ; \S 3]$. Модели с более сильными особенностями, чем логканонические (квази-логмногообразия и обобщенные логмногообразия), см. в $[1],[37]$.

КомментАРИй 1.8. Основные категории, с которыми мы работаем, - категория канонических логпар $(X, B)$ и категория логканонических логпар с их подкатегориями терминальных и соответственно логтерминальных логпар. В качестве морфизмов берутся рациональные 1-сдутия, т.е. модификации без раздутия дивизоров или, эквивалентно, обратная модификация не сдувает дивизоров. Категории канонических и терминальных логпар (Мори) определены корректно относительно ПММ или логПММ с $B=0$, где под корректностью понимается замкнутость относительно элементарных преобразований: экстремальных дивизориальных логсдутий и логфлипов, являюшихся соответственно дивизориальньми сдутиями из п. 1.1 с) и $D$-флипами из п. $1.1 \mathrm{~d})$ с $D_{i}=K+B$. То же самое проходит, если дивизориальные логсдутия не могут сдувать компонент границ, например, в категории подвижных логпар, когда граница составлена из общих дивизоров линейных систем без неподвижных компонент. Следуюший простой пример показывает, что в обшем случае дивизориальные логсдутия не сохраняют каноничность. Пусть $X=\mathbb{F}_{n}, n \geqslant 1,-$ линейчатая поверхность и $B=s_{n}$ - ее исключительное сечение. Тогда логпара $\left(\mathbb{F}_{n}, s_{n}\right)$ канонична и ее единственное элементарное преобразование - дивизориальное сдутие $\left(\mathbb{F}_{n}, s_{n}\right) \rightarrow\left(\mathbb{F}_{n}^{*}, 0\right)$ на конус $\mathbb{F}_{n}^{*}$ степени $n$, который при $n \geqslant 3(n \geqslant 2)$ имеет не каноническую (не терминальную) особенность в вершине.

Этот пример показьвает также, что важно иметь подходящее определение образа дивизораграницыпри бирациональном преобразовании (модификации) логпар. Стандартным является, конечно, понятие собственного образа $\chi_{*} B$ границы $B$ при бирациональном преобразовании $\chi: X \rightarrow Y$. Однако в логканонической категории естественным является понятие логобраза $B_{Y}^{\log }:=\chi_{*} B+\sum E_{i}$, где $E_{i}$ пробегает все исключительные простые дивизоры обратного преобразования $\chi^{-1}: Y \rightarrow X$. В дей- 
ствительности, как отмечено в [21; 2.7], [36; 1.1 .2$]$, существует много вариантов определения образа границы.

ОПРЕДЕЛЕНИЕ 1.9. Пусть $\chi:(X, B) \rightarrow\left(Y, B_{Y}^{\log }\right)$ - модификация полных нормальных многообразий $X$ и $Y$, где $B_{Y}^{\log }=B_{Y}+\sum E_{i}$ - логобраз $\mathbb{R}$-границы $B=\sum b_{i} D_{i}$, $B_{Y}=\chi_{*} B$ - собственный образ $B$, а $E_{i}$ - простые дивизоры на $Y$, раздутые $\chi$, т.е. исключительные для $\chi^{-1}$. Можно предполагать, что $K+B$ не обязательно lс и может быть не $\mathbb{R}$-Картье.

(i) Логпара $\left(Y, B_{Y}^{\log }\right)$ называется слабо логканонической моделью пары $(X, B)$, если $\left(Y, B_{Y}^{\log }\right)$ - слабо логканоническая модель и $a\left(D_{i}, Y, B_{Y}^{\log }\right) \geqslant a\left(D_{i}, X, B\right)=-b_{i}$ для всех $\chi$-исключительных простых дивизоров $D_{i}$ многообразия $X$. Логпара $\left(Y, B_{Y}\right)$ называется слабо канонической моделью пары $(X, B)$, если в этом определении логобраз $B_{Y}^{\log }$ заменить на собственньй образ $B_{Y}$, а слабую логканоничность модели заменить на слабую каноничность.

(ii) Логпара $\left(Y, B_{Y}^{\log }\right)$ называется логминимальной моделью пары $(X, B)$, если $\left(Y, B_{Y}^{\log }\right)$ - логминимальная модель и неравенства для дискрепантностей в (i) вьполнены в строгом смысле для всех $\chi$-исключительных простых дивизоров $D_{i}$ многообразия $X$. Логпара $\left(Y, B_{Y}\right)$ назьвается минимальной моделью пары $(X, B)$, если в этом определении логобраз $B_{Y}^{\log }$ заменить на собственный образ $B_{Y}$, а логминимальность модели заменить на минимальность.

(iii) Логпара $\left(Y, B_{Y}^{\log }\right)$ называется логканонической моделью пары $(X, B)$, если $\left(Y, B_{Y}^{\log }\right)$ - логканоническая модель и вьполнены неравенства для дискрепантностей из (i). Каноническая модель $\left(Y, B_{Y}\right)$ пары $(X, B)$, определяется аналогично слабо канонической с заменой слабой каноничности модели на каноничность.

Относительньй вариант этих понятий определяется стандартным образом. При этом модификация логпар меняется на модификацию

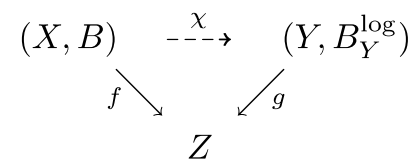

собственных морфизмов $f: X \rightarrow Z, g: Y \rightarrow Z$ или собственных относительных логпар $f:(X, B) \rightarrow Z, g:\left(Y, B_{Y}^{\log }\right) \rightarrow Z$. Так, например, морфизм $g:\left(Y, B_{Y}^{\log }\right) \rightarrow Z$ называется относительною слабо логканонической моделью морфизма $f:(X, B) \rightarrow Z$, если $g$ - относительная слабо логканоническая модель и $a\left(D_{i}, Y, B_{Y}^{\log }\right) \geqslant a\left(D_{i}, X, B\right)=$ $-b_{i}$ для всех $\chi$-исключительных простых дивизоров $D_{i}$ многообразия $X$.

ЗАмЕчАния 1.10. (i) В версии Коллара неравенства при определении логминимальной модели в (ii) определения 1.9 - не строгие [21; 2.15 .1$)]$. В его определении возможны сдутия приведенных компонент гранищы, когда они сохраняют логминимальность. Например, если $(-1)$-кривую $D_{0}$ на неособой поверхности $X$ трансверсально и просто пересекают две неособые кривые $D_{1}, D_{2}$ и $D_{1} \cap D_{2}=\varnothing$, то сдутие $\chi: X \rightarrow Y$ кривой $D_{0}$ дает обыкновенную двойную точку образа $B_{Y}=\chi_{*} B$, где $B=\sum D_{i}$. Модель $\left(Y, B_{Y}=B_{Y}^{\log }\right) \rightarrow Y$ морфизма $(X, B) \rightarrow Y$ логминимальна в смысле Коллара над окрестностью точки $\chi\left(D_{0}\right) \in Y$, но не в определении 1.9 (ii).

Аналогичное неравенство для минимальных моделей выполнено автоматически и всегда строгое: $a\left(D_{i}, Y, B_{Y}\right)>0 \geqslant-b_{i}$, поскольку $B$ - граница. 
(ii) В действительности, как мы увидим ниже, если $g:\left(Y, B_{Y}^{\log }\right) \rightarrow Z$ - относительная слабо логканоническая модель собственного морфизма $f:(X, B) \rightarrow Z$ с $\mathbb{R}$-Картье $K+B$, то $a\left(F, Y, B_{Y}^{\log }\right) \geqslant a(F, X, B)$ для всех простых b-дивизоров $F$ многообразия $X$; неравенство строгое, если $\left(Y, B_{Y}^{\log }\right) \rightarrow Z$ - относительная логканоническая модель и модификация $\chi$ не определена в общей точке центра $c_{X} F$.

Аналогичное верно для канонических моделей пар.

(iii) Основная проблема логПММ - показать, что результирующая модель существует для всякого собственного морфизма $f: X \rightarrow Y$. Удовлетворительные результаты получены пока только для $\operatorname{dim} X \leqslant 3$. Некоторые общие результаты такие, как теорема о конусе, теорема о необращении в нуль, теорема о свободе линейной системы, теорема о стягивании, получены в любой размерности (см. [17], [33], [21], [22]). Открытыми остаются пока проблемы сушествования логфлипов в размерности $\geqslant 5$ и логобрьва в размерности $\geqslant 4$ (см. об этом подробнее в [37; Section 1], [39]), а также полуобильность $[36 ; 2.6]$ в размерности $\geqslant 4$.

Сформулируем основные гипотезы в логПММ.

ГиПотеЗА 1.11 (сушествование логфлипов). Пусть $(X, B)$ - lt логпара с $\mathbb{Q}$-факториальньм $X, \varphi: X \rightarrow Y$ - малое экстремальное $(K+B)$-сдутие. Тогда логфлип существует, т.е. сушествует диаграмма (1.1) со свойствами (i)-(iii) для дивизоров $D_{i}=$ $K+B, D_{i}^{+}=K_{X_{i}^{+}}+B^{+}$и $B^{+}=g_{i *} B$.

Как уже отмечалось в замечаниях 1.7 (ii) и 1.10 (iii), гипотеза доказана в размерности $\operatorname{dim} X \leqslant 4$ (см. [37; Corollary 1.8, 1.12 History]).

ГипОТЕЗА 1.12 (обрыв логфлипов). В условиях гипотезы 1.11 любая последовательность логфлипов (1.2) для $K+B$ обрывается на конечном шаге:

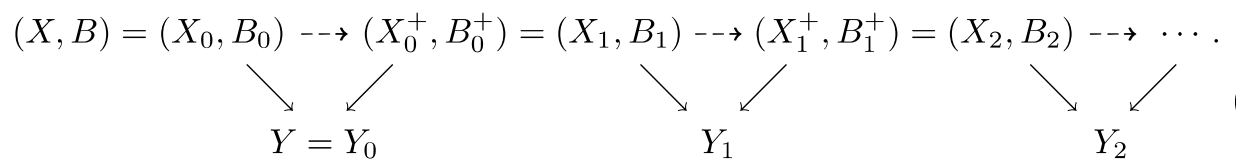

Эта гипотеза доказана для $\operatorname{dim} X \leqslant 3$ (см. [33; (2.17)], [12], [36; 5.2]).

УСЛОВНАЯ ТЕОРЕМА 1.13 (по модулю гипотез 1.11 и 1.12). Для любой логпары $(X, B)$ и, более общо, в относительной ситуачии для собственного морфизма $X \rightarrow Z$ существует модификация

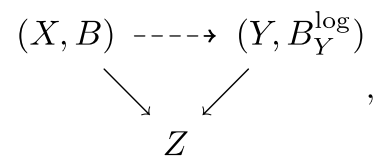

где пара $\left(Y, B_{Y}^{\log }\right) \rightarrow Z$ проективна над $Z, \mathbb{Q}$-факториальна и либо является логминимальной моделью для $(X, B) \rightarrow Z$, либо имеет структуру лограсслоения Мори $\left(Y, B_{Y}^{\log }\right) \rightarrow S$ над $Z$.

ДокАЗАТЕльство. После логразрешения можно предполагать, что логпара $(X, B)$ как в гипотезе 1.11. Затем применяем логПММ. Подробности см. в [25; 11-1-4], [17; Introduction], [36; Section 5] (для обобщений на случай логканонических пар $(X, B)$ и $\mathbb{R}$-границ $B)$. 
ГиПотЕЗА 1.14 (существование эффективного логканонического дивизора). Пусть $\left(Y, B_{Y}\right) \rightarrow Z$ - слабо логканоническая модель с $\mathbb{Q}$-гранищей $B_{Y}$. Тогда $\left|m\left(K_{Y}+B_{Y}\right)\right|$ $\neq \varnothing$ над $Z$ для некоторых натуральных $m \gg 0$, т.е. $\kappa\left(Y / Z, B_{Y}\right) \geqslant 0$.

Для $\operatorname{dim} X \leqslant 3$ эта гипотеза доказана в [19]. В размерностях $\geqslant 4$ вопрос пока остается открытым.

УСЛовная ТЕОРемА 1.15 (характеризация через логкодаирову размерность по модулю гипотез $1.11,1.12$ и 1.14). Собственная lс логпара $(X, B) \rightarrow Z$ c $\mathbb{Q}$-границей $B_{Y}$ имеет логминимальную модель тогда и только тогда, когда $\kappa(X / Z, B) \geqslant 0, u$ - лограсслоение Мори тогда и только тогда, когда $\kappa(X / Z, B)=-\infty$.

Эта теорема пока доказана безусловно только для $\operatorname{dim} X \leqslant 3$ (см. [19], [36; 2.4,2.7], а также [13], [21; Sections 11-14]).

ГиПотЕЗА 1.16 (полуобильность). Пусть $\left(Y, B_{Y}\right) \rightarrow Z$ - слабо логканоническая модель. Тогда дивизор $K_{Y}+B_{Y}$ полуобилен над $Z$. Для $\mathbb{Q}$-границы $B_{Y}$ это эквивалентно тому, что линейная система $\left|m\left(K_{Y}+B_{Y}\right)\right|$ над $Z$ свободна для некоторых натуральных $m \gg 0$.

Как и гипотеза 1.14, эта гипотеза доказана пока только для $\operatorname{dim} X \leqslant 3$ (см. [19], [36; 2.7], а также [13], [21; Sections 11-14]).

ЗАмЕчАния 1.17. (i) В случае сn особенностей гипотеза 1.12 доказана также и в размерности 4 [39; Example 9], так что для завершения ПММ для $\operatorname{dim} X=4$ осталось установить гипотезы 1.14 и 1.16 в этой размерности.

(ii) В случае $\mathbb{Q}$-границы $B_{Y}$, если верна гипотеза 1.16 , то линейная система $\left|m\left(K_{Y}+B_{Y}\right)\right|$ для некоторых натуральных $m \gg 0$ задает расслоение Иитаки $I\left(Y, B_{Y}\right): Y \rightarrow Y_{\text {can }}$ на нормальное проективное многообразие $Y_{\text {can }}$, характеризуюшееся свойством: для всякой кривой $C \subset Y$

$$
I\left(Y, B_{Y}\right)(C)=\text { pt. } \Longleftrightarrow\left(K_{Y}+B_{Y}\right) C=0 .
$$

По определению $\kappa\left(Y, B_{Y}\right)=\operatorname{dim} Y_{\text {сап и }}$

$$
Y_{\text {can }} \simeq \operatorname{Proj} \bigoplus_{m \geqslant 0} H^{0}\left(Y, m\left(K_{Y}+B_{Y}\right)\right) .
$$

Из доказательства теоремы 1.13 , теоремы 1.15 и гипотезы 1.16 с $B_{Y}=B_{Y}^{\log }$ следует, что для любой логпары $(X, B)$ такой, как в теореме 1.15, логканоническое кольцо

$$
\mathscr{R}(X, B)=\mathscr{R}_{X}(K+B)=\bigoplus_{m \geqslant 0} H^{0}(X, m(K+B))=\bigoplus_{m \geqslant 0} H^{0}\left(Y, m\left(K_{Y}+B_{Y}^{\text {log }}\right)\right)
$$

конечно порождено (в случае $\kappa(X, B)=-\infty$ это кольцо $\mathscr{R}(X, B)=k$-основное поле). Оно является важньм бирациональным инвариантом пары $(X, B)$.

(iii) Геометрическим, но трудно проверяемым критерием сушествования на $(X, B)$ бирациональной структуры лограсслоения Мори является по модулю гипотез 1.11 и 1.12 следующее свойство: существует открытое непустое подмножество $U \subset X$ такое, что через каждую точку $x \in U$ проходит кривая $C_{x}$ с $(K+B) C_{x}<0$.

Ниже мы обсудим некоторые свойства логмоделей в тех случаях, когда они существуют. 


\section{§. Канонические и логканонические модели}

2.1. Канонические особенности - это особенности, которые появляются на канонических моделях многообразий общего типа. Пусть $X$ - полное неособое алгебраическое многообразие общего типа (т.е. кодаировой размерности $\kappa(X)=\operatorname{dim} X)$ и

$$
\mathscr{R}(X)=\mathscr{R}_{X}(K)=\bigoplus_{m \geqslant 0} H^{0}(X, m K)
$$

- каноническое кольцо с естественным умножением сечений. Каноническая модель

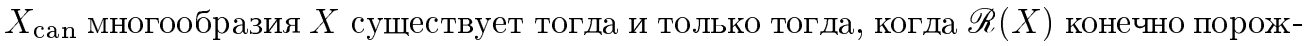
дено как $k=H^{0}\left(X, \mathscr{O}_{X}\right)$-алгебра, и тогда $X_{\text {can }}=\operatorname{Proj} \mathscr{R}(X)$. Отсюда получаем, что каноническая модель единственна в своем бирациональном классе. Ясно также, что она $\mathbb{Q}$-горенштейнова или $K_{X_{\text {can }}} \mathbb{Q}$-Картье, т.е. $n K_{X_{\text {сап }}}$ - дивизор Картье для некоторого целого $n \neq 0$. Хорошо известно, что в размерности 2 особенности $X_{\text {can }}$ - это в точности двойные рациональные (или, как их часто назьвают, дювалевские).

Канонические особенности в любой размерности были определены Ридом [30]. Там же показано, что в точности такие особенности появляются на канонических моделях многообразий обшего типа.

В логкатегории свойство обильности для логканонического дивизора $K+B$ обобщается до численной обильности, которая совпадает с обычной для $\mathbb{Q}-$ Картье дивизоров. Соответственно каноничность особенностей обобщается до логканоничности.

Наша ближайшая цель - изучить вопрос о единственности логканонической модели и канонической модели, в частности - для подвижных логnар, т.е. для пар $(X, H)$, где $H$ - общий дивизор из $\sum r_{i} \mathscr{M}_{i}, r_{i} \in[0,1), \mathscr{M}_{i}$ - линейные системы без неподвижных компонент; обычно также предполагается, что $H \neq 0$, что эквивалентно подвижности одной из линейных систем $\mathscr{M}_{i}$ с $r_{i}>0$ (см. [4]). Начнем со следуюшего утверждения.

ЛЕмма 2.2 (об отрищательности [34; 1.1], [21;2.19]). Пусть $f: Y \rightarrow X-c \partial y т u е$, $Y$ нормально и $E_{i} \subset Y-f$-исключительные простые дивизоры. Предположим, что имеет место относительная численная $f$-эквивалентность $\mathbb{R}$-дивизоров

$$
\sum c_{i} E_{i} \equiv N+G
$$

где $N$ - численно $f$-әффективный дивизор и $G$ - әффективный дивизор, не содержащий $f$-исключительных компонент. Тогда все $c_{i} \leqslant 0 u c_{i}<0$, если $N$ не является численно тривиальным на $f^{-1} f\left(E_{i}\right)$ над $X$.

ДоКАЗАТЕЛЬСтво. Оно хорошо известно и содержится, например, в [34; с. 107], $[21 ; 2.19],[25 ; 13-1-4]$. Основная идея - сведение к случаю поверхностей и использование леммы об отрицательной определенности формы пересечения компонент сдуваемых кривых.

Вот основной результат этого параграфа.

ТЕОРЕма 2.3 (единственность канонической и логканонической моделей).

(i) Каноническая модель пары (в частности, для подвижной логпары) единственна, если она существует.

(ii) Логканоническая модель для любой логпары единственна, если она существует. 
ДоКАЗАТЕЛЬСТво. Оно проходит единым образом для (i) и (ii) с использованием леммы об отрищательности. Отметим, что всякая каноническая модель подвижной логпары $\left(X, \sum r_{i} \mathscr{M}_{i}\right)$ по определению есть каноническая модель пары $(X, H)$ для общего дивизора $H \in \sum r_{i} \mathscr{M}_{i}$; подвижная пара однозначно восстанавливается по общему $H$. Для простоты обозначений мы опускаем базисное многообразие $Z$, над которым рассматриваются все пары; $Z$ = pt. - точка отвечает, как обычно, глобальному случаю.

Предположим, что существуют две канонические модели $\left(Y_{1}, B_{1}=B_{Y_{1}}\right)$ и $\left(Y_{2}, B_{2}=B_{Y_{2}}\right)$ пары $(X, B)$. Рассмотрим домик Хиронаки, разрешаюший модификацию моделей $\chi:\left(Y_{1}, B_{1}\right) \rightarrow\left(Y_{2}, B_{2}\right)$,

$$
\left(Y_{1}, B_{1}\right) \underset{-\alpha}{\alpha} \searrow_{\left(Y_{2}, B_{2}\right)}^{\left(W, B_{W}\right)}
$$

где $\left(W, B_{W}\right)$ - логразрешение как $\left(Y_{1}, B_{1}\right)$, так и $\left(Y_{2}, B_{2}\right)$, а $\alpha$ и $\beta$ - соответствуюшие сдутия. При подходящем выборе канонических дивизоров $K_{W}, K_{Y_{1}}$ и $K_{Y_{2}}$ можно записать

$$
K_{W}+B_{W}=\alpha^{*}\left(K_{Y_{1}}+B_{1}\right)+\sum c_{i} E_{i}=\beta^{*}\left(K_{Y_{2}}+B_{2}\right)+\sum c_{i}^{\prime} E_{i}^{\prime},
$$

где $E_{i}\left(E_{i}^{\prime}\right)$ - исключительные простые дивизоры для $\alpha$ (соответственно $\left.\beta\right)$.

Утверждается, что все $c_{i} \geqslant 0$ и $c_{i}^{\prime} \geqslant 0$. По определению $B_{1}$ и $B_{W}$ - собственные преобразования $B=\sum b_{i} D_{i}$. Откуда $B_{1}=\alpha_{*} B_{W}$ и $B_{W}=\left(B_{1}\right)_{W}+\sum b_{i} E_{i}$, где $\left(B_{1}\right)_{W}-$ собственное преобразование $B_{1}$ и

$$
b_{i}= \begin{cases}b_{i}, & \text { если } E_{i}-\text { собственное преобразование } D_{i}, \\ 0 & \text { в противном случае }\end{cases}
$$

(в логкатегории 0 меняется на 1 ). Поэтому для любого исключительного дивизора $E_{i}$

$$
c_{i}=a\left(E_{i}, Y_{1}, B_{Y_{1}}\right)+b_{i} \geqslant 0
$$

в силу каноничности модели. Это же доказательство работает для $c_{i}^{\prime}$.

Из формулы (2.2) получаем соотношение

$$
\sum\left(c_{i}^{\prime}-c_{i}\right) E_{i}^{\prime}=\alpha^{*}\left(K_{Y_{1}}+B_{1}\right)-\beta^{*}\left(K_{Y_{2}}+B_{2}\right)+\sum c_{i} E_{i},
$$

где первое суммирование ведется по исключительным дивизорам сдутия $\beta$, а второе по исключительньм дивизорам сдутия $\alpha$, которые не исключительны для $\beta$. В частности, последняя сумма эффективна, так как $c_{i} \geqslant 0$. С другой стороны, $\alpha^{*}\left(K_{Y_{1}}+B_{1}\right)-$ $\beta^{*}\left(K_{Y_{2}}+B_{2}\right)$ численно $\beta$-эффективен в силу численной эффективности $\alpha^{*}\left(K_{Y_{1}}+B_{1}\right)$, $K_{Y_{1}}+B_{1}$ и численной $\beta$-тривиальности $\beta^{*}\left(K_{Y_{2}}+B_{2}\right)$. Значит, по лемме $2.2 c_{i} \geqslant c_{i}^{\prime}$ надо всеми $\beta$-исключительными дивизорами $E_{i}^{\prime}$, а по неотрицательности $c_{i}-$ всюду. Аналогично $c_{i}^{\prime} \geqslant c_{i}$. Откуда $c_{i}=c_{i}^{\prime}$ всюду.

Снова по формуле (2.2)

$$
\alpha^{*}\left(K_{Y_{1}}+B_{1}\right)=\beta^{*}\left(K_{Y_{2}}+B_{2}\right)
$$


и $\chi:\left(Y_{1}, B_{1}\right) \stackrel{\sim}{\rightarrow}\left(Y_{2}, B_{2}\right)-$ изоморфизм моделей в силу численной обильности $K_{Y_{1}}+B_{1}$ и $K_{Y_{2}}+B_{2}$. Действительно, если отображение $\chi$ имеет точки неопределенности, то на $Y_{1}$ должна существовать точка, образ которой на $Y_{2}$ имеет размерность $>0$. Значит, на $W$ найдется кривая $C$ такая, что $\beta(C) \subset Y_{2}$ - кривая и $\alpha(C) \subset Y_{1}$ - точка неопределенности. Но это невозможно по (2.3), что доказьвает утверждение (i) теоремы (аналогично доказывается (ii); см. также доказательство предложения 2.4 в [36]).

Аналогичным образом из леммы 2.2 выводится следуюшая монотонность (см. $[34 ; 1.5],[21 ;$ Section 2$])$.

Лемма 2.4. Пусть $f:(X, B) \rightarrow Z-$ собственная относительная логпара такая, что $K+B \mathbb{R}-$ Картье, и $g:\left(Y, B_{Y}^{\log }\right) \rightarrow Z$ - ее слабо логканоническая модель. Тогда для любого простого b-дивизора $Е$ многообразия $X$ (равносильно, многообразия $Y$ )

$$
a\left(E, Y, B_{Y}^{\log }\right) \geqslant a(E, X, B) .
$$

Более того, если $g$ - логканоническая модель для $f$ (в частности, $K_{Y}+B_{Y}^{\log }$ обилен над $Z)$ и модификачия $\chi: X \rightarrow Y$ не определена в общей точке $\mathrm{c}_{X} E$, то

$$
a\left(E, Y, B_{Y}^{\log }\right)>a(E, X, B) .
$$

ДОкАЗАТЕЛЬСТво $[34 ;(1.5 .6)],[21 ;(2.23 .3)]$. Проводим в зависимости от исключительности или неисключительности $E$ на $X$.

Случай I. Если b-дивизор $E$ не исключителен на $X$, то требуемое неравенство получается по определению слабо логканонической модели (см. 1.9 (i)). (При этом модификация определена в общей точке $\mathrm{c}_{X} E=E$.)

Случай II. Предположим теперь, что b-дивизор $E$ исключителен на $X$, и рассмотрим его дивизориальное разрешение на многообразии $W$ как в диаграмме (2.1) с $\left(Y_{1}, B_{1}\right)=(X, B)$ и $\left(Y_{2}, B_{2}\right)=\left(Y, B_{Y}^{\log }\right)$. Тогда по определению дискрепантностей

$$
\alpha^{*}(K+B)+\sum a\left(D_{i}, X, B\right) D_{i}=K_{W}=\beta^{*}\left(K_{Y}+B_{Y}^{\log }\right)+\sum a\left(D_{i}, Y, B_{Y}^{\log }\right) D_{i} .
$$

Разделяя суммирование по всем дивизорам $D_{i}$ на $W$ на 2 части: по исключительным $E_{i}$ и не исключительныv $D_{i}$ на $X$, получаем соотношение

$$
\begin{aligned}
& \sum\left(a\left(E_{i}, X, B\right)-a\left(E_{i}, Y, B_{Y}^{\log }\right)\right) E_{i} \\
& \quad=\beta^{*}\left(K_{Y}+B_{Y}^{\log }\right)-\alpha^{*}(K+B)+\sum\left(a\left(D_{i}, Y, B_{Y}^{\log }\right)-a\left(D_{i}, X, B\right)\right) D_{i},
\end{aligned}
$$

в котором дивизор $\beta^{*}\left(K_{Y}+B_{Y}^{\log }\right)-\alpha^{*}(K+B)$ численно эффективен над $X$ в силу численной эффективности $\beta^{*}\left(K_{Y}+B_{Y}^{\log }\right)$ над $Z$ и численной тривиальности $\alpha^{*}(K+B)$ над $X$. С другой стороны, дивизор $\sum\left(a\left(D_{i}, Y, B_{Y}^{\log }\right)-a\left(D_{i}, X, B\right)\right) D_{i}$ эффективен по случаю І. Отсюда по лемме 2.2 вытекает эффективность дивизора $\sum\left(a\left(E_{i}, Y, B_{Y}^{\log }\right)-\right.$ $\left.a\left(E_{i}, X, B\right)\right) E_{i}$ и требуемое неравенство, поскольку $E$ отождествляется с одним из исключительных дивизоров $E_{i}$.

Предположим дополнительно, что $g$ - логканоническая модель для $f$ и модификация $\chi$ не определена в общей точке центра $c_{X} E$. Тогда дивизор $\beta^{*}\left(K_{Y}+B_{Y}^{\log }\right)$ не является численно тривиальным на $\alpha^{-1} c_{X} E$ по обильности $K_{Y}+B_{Y}^{\log }$ над $Z$. Стало быть, требуемое строгое неравенство вытекает снова из леммы 2.2 . 
ПРЕДЛОЖЕНИЕ 2.5. Пусть $g:\left(Y, B_{Y}^{\log }\right) \rightarrow Z-$ слабо логканоническая модель собственного морфизма $f:(X, B) \rightarrow Z$ и $\chi: X \rightarrow Y-$ соответствующая модификачия. Тогда

(i) $f:(X, B) \rightarrow Z$ не имеет лограсслоения Мори и даже Фано; при этом особенности пары $(X, B)$ не важнны, требуется лишь $\mathbb{R}-$ Картье свойство $K+B$ и не 0-мерность общего слоя расслоения Фано;

(ii) если $(X, B)$ lc и модель $g$ логканонична, то $\chi^{-1}$ не имеет исключительных дивизоров;

(iii) если $g_{\text {can }}: H_{\text {can }} \rightarrow Z$ - логканоническая модель Иитаки любой слабо логканонической модели $\left(Y_{1}, B_{1}^{\log }=B_{Y_{1}}^{\log }\right) \rightarrow Z$ морфизма $f$, то существует единственный морфизм $\rho: Y \rightarrow Y_{\text {can }}$ над $Z$, при әтом $\rho=I\left(Y / Z, B_{Y}^{\log }\right)$ - тоже расслоение Иитаки, т.е. и модель Иитаки единственна;

(iv) более того, логканоническая модель Иитаки $Y_{\text {can }} \rightarrow Z$ существует тогда и только тогда, когда $K_{Y}+B_{Y}^{\log }$ полуобилен над $Z$, при этом $H_{\text {сап }} \rightarrow Z$ относительная логканоническая модель $f$ ровно тогда, когда $K_{Y}+B_{Y}^{\log }$ обгемен над $Z$;

$(\mathrm{v})$ если $K+B \mathrm{lc}, \mathbb{Q}$-Картье и облемен над $Z$, то каждая из моделей логканоническая и Иитаки $g_{\mathrm{can}}: Y_{\mathrm{can}} \rightarrow Z$ существует тогда и только тогда, когда алгебра $f_{*} \mathscr{O}_{X}-\mathcal{м о д у л е и ̆ ~}$

$$
\bigoplus_{m \geqslant 0} f_{*} \mathscr{O}_{X}(m(K+B))
$$

конечно порождена, при этом

$$
Y_{\text {can }}=\operatorname{Proj} \bigoplus_{m \geqslant 0} f_{*} \mathscr{O}_{X}(m(K+B))
$$

обе модели совпадают, когда существуют.

Аналогичные утверждения верны и без лог и даже более того:

(vi) любые две минимальные модели морфизма $f$ изоморфны в коразмерносmu 1 ;

(vii) всякая строго минимальная и в то же время каноническая модель изоморфна любой слабо канонической модели $f$.

В лемме 2.4 и всех утверждениях предложения пару $(X, B)$ можно заменить на любую другую логпару $\left(Y^{\prime}, B_{Y^{\prime}}^{\log }\right)$ и в случае без лог на $\left(Y^{\prime}, B_{Y^{\prime}}\right)$ с теми же условиями, что, например, упрошает доказательство предложения (iii) ниже.

Доказательство. (i) Рассмотрим диаграмму $(2.1)$ с $\left(Y_{1}, B_{1}\right)=(X, B)$ и $\left(Y_{2}, B_{2}\right)=\left(Y, B_{Y}^{\log }\right)$. Пусть $(X, B) \rightarrow S \rightarrow Z$ - относительное лограсслоение Мори или Фано со стягиванием $\varphi: X \rightarrow S$ над $Z$. По определению и нашим предположениям $\operatorname{dim} S<\operatorname{dim} X$ и дивизор $-(K+B)$ численно обилен над $S$. Покажем, что это невозможно.

По лемме о монотонности 2.4 в силу логканоничности модели $\left(Y, B_{Y}^{\log }\right) \rightarrow Z$ диви3оp

$$
\alpha^{*}(K+B)-\beta^{*}\left(K_{Y}+B_{Y}^{\log }\right)=\sum\left(a\left(D_{i}, Y, B_{Y}^{\log }\right)-a\left(D_{i}, X, B\right)\right) D_{i} \geqslant 0
$$


Следовательно, он неотрицательно пересекает достаточно общие кривые, например, в слоях стягивания $\varphi \circ \alpha$. Но по конструкции такие кривые дивизор $\alpha^{*}(K+B)-$ $\beta^{*}\left(K_{Y}+B_{Y}^{\log }\right)$ пересекает отрицательно. Противоречие.

(ii) Воспользуемся обозначениями и результатом леммы 2.4. Пусть $E-\chi^{-1}$-исключительньй дивизор. Тогда по лемме $a(E, X, B)<a\left(E, Y, B_{Y}^{\log }\right)=-1$ (последнее равенство по определению логобраза). Но это противоречит логканоничности пары $(X, B)$.

(iii) В общем случае неверно, что каждая из логпар $\left(Y_{1}, B_{1}^{\log }=B_{Y_{1}}^{\log }\right) \rightarrow Z$ и $\left(Y, B_{Y}^{\log }\right) \rightarrow Z$ является слабо логканонической моделью другой. Действительно, простая компонента $D_{i}$ границы $B$ может быть не исключительна на $Y$, но исключительна на $Y_{1}$. Если при этом $b_{i}<1$, то $B_{Y}^{\log } \neq\left(B_{1}^{\log }\right)_{Y}^{\log }$. Однако для подходящей теории преобразования гранищ (см. замечание о преобразовании границ после комментария 1.8) утверждение о том, что каждая из логпар $\left(Y_{1}, B_{1}^{\log }\right) \rightarrow Z$ и $\left(Y, B_{Y}^{\log }\right) \rightarrow Z$ является слабо логканонической моделью другой, и лемма 2.4 в этой более обшей ситуации верны. Для последней важны правильные обобщения неравенств в определении 1.9 (ii) слабо логканонической модели. Тем самьм по лемме 2.4 все $a\left(E, Y_{1}, B_{1}^{\log }\right)=$ $a\left(E, Y, B_{Y}^{\log }\right)$ (см. подробности в доказательстве утверждения 2.4 .2 в [36]). Отсюда, как и в доказательстве теоремы 2.3 , получаем, что $\rho=I\left(Y, B_{Y}^{\log }\right): Y \rightarrow H_{\text {can }}-$ морфизм, задаваемый дивизором $K_{Y}+B_{Y}^{\log }$ (cp. с [36; 2.4.3]).

(iv) Если $K_{Y}+B_{Y}^{\log }$ полуобилен над $Z$, то сушествуют стягивание $I=I\left(Y / Z, B_{Y}^{\log }\right)$ :

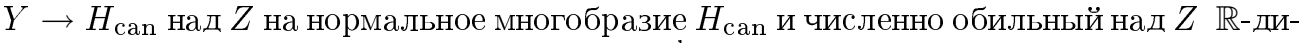

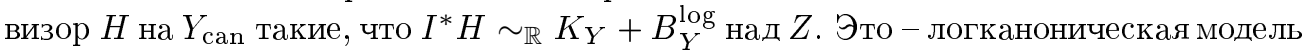
Иитаки. Обратное верно по определению. Расслоение Иитаки $I$ бирационально ровно тогда, когда $K_{Y}+B_{Y}^{\log }$ объемен, при этом модель $\left(Y_{\text {can }}, B_{Y_{\text {can }}}^{\log }\right) \rightarrow Z$ логканонична. Сдутие $I$ крепантно в силу численной тривиальности $K_{Y}+B_{Y}^{\log }$ над $Z$, а потому $K_{H_{\text {can }}}+B_{H_{\text {can }}}^{\log }$ lc. Также по численной тривиальности $K_{Y}+B_{Y}^{\log }=I^{*}\left(K_{H_{\text {can }}}+B_{H_{\text {can }}}^{\log }\right)$ и $K_{H_{\text {can }}}+B_{H_{\text {can }}}^{\log } \sim_{\mathbb{R}} H$ обилен. Обратно, логканоническая модель является моделью Иитаки по (iii) с $Y_{1}=H_{\text {can }}$.

(v) Утверждения о логканонической модели используют стабилизацию подвижной части кратных логканонических дивизоров при вьполнении конечной порожденности (ср. предельньй критерий 5.21 ниже, подробности в доказательстве [30; $1.2(\mathrm{II})]$ для канонического дивизора и $[37 ; 3.18]$ в обшем случае). При этом если $\left(Y, B_{Y}^{\log }\right) \rightarrow Z-$ логканоническая модель, то $g_{\text {саn }}=g: H_{\text {сап }}=Y \rightarrow Z$-ее модель Иитаки по (iii)-(iv). Тем самым получаем сушествование обеих моделей по конечной порожденности алгеб-

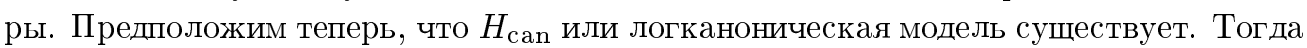
по (iv) $K_{Y}+B_{Y}^{\log }$ - полуобильньй $\mathbb{Q}$-дивизор. Композиция $I \circ \chi: X--\rightarrow Y \rightarrow H_{\text {сап ин- }}$ дуцирует естественные квазиизоморфизмы градуированных алгебр (см. абзац перед предложением 5.4 и $[37 ; 4.3])$ и изоморфизмы их проективных спектров:

$$
\begin{aligned}
Y_{\text {can }} & =\operatorname{Proj} \bigoplus_{m \geqslant 0} g_{\text {can } *} \mathscr{O}_{H_{\text {can }}}(m H) \\
& =\operatorname{Proj} \bigoplus_{m \geqslant 0} g_{*} \mathscr{O}_{Y}\left(m\left(K_{Y}+B_{Y}^{\log }\right)\right)=\operatorname{Proj} \bigoplus_{m \geqslant 0} f_{*} \mathscr{O}_{X}(m(K+B)),
\end{aligned}
$$

последний изоморфизм использут lс пары $(X, B)$, где $H$ - теперь $\mathbb{Q}$-дивизор в (iv); 
при этом конечная порожденность средней алгебры влечет конечную порожденность последней.

(vi) Пусть $\left(Y_{1}, B_{1}=B_{Y_{1}}\right) \rightarrow Z$ и $\left(Y_{2}, B_{2}=B_{Y_{2}}\right) \rightarrow Z$ - две минимальные модели $f$. Предположим, что их модификашия $\chi: Y_{1} \rightarrow Y_{2}$ сдувает дивизор $E \subset Y_{1}$. Тогда по версии без лог леммы $2.4 a\left(E, Y_{2}, B_{2}\right) \leqslant a\left(E, Y_{1}, B_{1}\right)=-$ mult $_{E} B_{1} \leqslant 0$, что противоречит минимальности модели $\left(Y_{2}, B_{2}\right)$. Аналогично, обратная модификация $\chi^{-1}$ не сдувает дивизоров.

(vii) Доказьвается аналогично.

КомментАРИй 2.6. Метод доказательства теоремы 2.3, основанньй на лемме об отрицательности 2.2 , аналогичен методу доказательства классического неравенства Нётера-Фано в бирациональной теории расслоений Мори. Обычно линейная система, задаюшая нетривиальное (т.е. не являюшееся изоморфизмом) бирациональное отображение между такими расслоениями, имеет базисное множество достаточно большой кратности - максимальную особенность. Напротив, отсутствие в линейной системе максимальной особенности можно всегда интерпретировать как каноничность особенностей подходящей пары. Если эта пара оказьвается к тому же слабо канонической моделью, то исходное бирациональное отображение, задаваемое линейной системой, является изоморфизмом. В этой формулировке неравенство Нётера-Фано используется как критерий остановки алгоритма разложения бирационального отображения на линки и может быть интерпретировано как единственность слабо канонической модели в такой ситуации.

Рассмотрим бирациональное отображение между двумя расслоениями Мори (не сохраняюшее, вообше говоря, структуры расслоения)

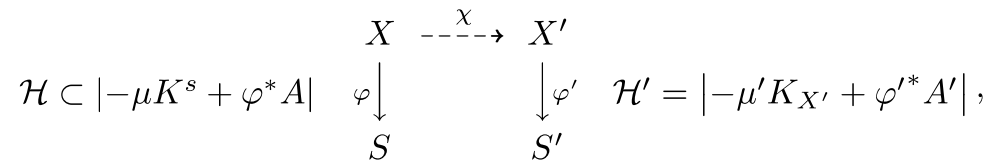

где многообразия $X, S^{\prime}$ проективны, $\mathscr{H}^{\prime}$ - полная очень обильная линейная система, $\mu^{\prime} \in \mathbb{Z}_{>0}, A^{\prime} \in \operatorname{Div}\left(S^{\prime}\right)$ - очень обильный дивизор на $S^{\prime}, \mathscr{H}=\chi_{*}^{-1} \mathscr{H}^{\prime}-$ собственный образ на $X$ линейной системы $\mathscr{H}^{\prime}, \mu \in \mathbb{Q}_{>0}, K^{s}$ - полуканонический дивизор, т.е. канонический с точностью до $\mathbb{Q}$-линейной эквивалентности (если $X$ неособо на общем слое $\varphi$ и группа Пикара последнего порождена каноническим дивизором, то $\mu \in \mathbb{Z}_{>0}$ и $K^{s}$ можно заменить на $\left.K\right), A \in \operatorname{Div}(S) \otimes \mathbb{Q}$ - $\mathbb{Q}$-дивизор. Рассмотрим две (подвижные) логпары $\left(X, \frac{1}{\mu} H\right)$ и $\left(X^{\prime}, \frac{1}{\mu^{\prime}} H^{\prime}\right)$, где $H \in \mathscr{H}$ и $H^{\prime}=H_{X^{\prime}} \in \mathscr{H}^{\prime}$ - общие дивизоры линейных систем без неподвижных компонент. По выбору системы $\mathscr{H}^{\prime}$ пара $\left(X^{\prime}, \frac{1}{\mu^{\prime}} H^{\prime}\right)$ является минимальной моделью пары $\left(X, \frac{1}{\mu^{\prime}} H\right)$ при $\mu^{\prime} \geqslant 2$. Точнее, для всех исключительных простых дивизоров $E$ любого разрешения $W$ (и любого замкнутого подмножества в $X^{\prime}$ ) дискрепантности $a\left(E, X^{\prime}, H^{\prime}\right)=a\left(E, X^{\prime}, 0\right)>0$ (и кратность $H^{\prime}$ в любой общей точке замкнутого подмножества равна 0) для достаточно общего дивизора $H^{\prime}$ свободной линейной системы (выбор его зависит от разрешения и замкнутого подмножества), а $\left(X^{\prime}, 0\right)$ имеет не более чем терминальные особенности. Отсюда получаем $\operatorname{trm}$ пары $\left(X^{\prime}, \frac{1}{\mu^{\prime}} H^{\prime}\right)$ и $H^{\prime}=H_{X^{\prime}}$. Кроме того, $K_{X^{\prime}}+\frac{1}{\mu^{\prime}} H^{\prime} \sim_{\mathbb{Q}} K_{X^{\prime}}-K_{X^{\prime}}+$ $\frac{1}{\mu^{\prime}} \varphi^{\prime *} A^{\prime}=\frac{1}{\mu^{\prime}} \varphi^{\prime *} A^{\prime}$ численно эффективен на $X^{\prime}$, так как $A^{\prime}$ обилен на $S^{\prime} ;$ при этом $H^{\prime} \neq 0$. 
Для пары $(X, H)$ определим канонический порог

$$
c(X, H):=\max \{t \in \mathbb{Q} \mid K+t H \mathrm{cn}\} .
$$

Напомним, что сn означает, что $\operatorname{dis}(X, t H) \geqslant 0$, т.е. дискрепантности $a(E, X, t H)$ неотрицательны для каждого простого b-дивизора $E$ многообразия $X$ с центром с $X$ коразмерности $\geqslant 2$. Для целого $H>0$ порог $c(X, H) \leqslant 1$ и его значение $c(X, H)<1$ можно вьчислить явно следующим образом. Пусть $\alpha: W \rightarrow X$ - достаточно нетривиальное логразрешение пары $(X, H)$, a $E_{i}$ - исключительные простые дивизоры для $\alpha$. В этом случае

$$
\begin{aligned}
& K_{W}=\alpha^{*} K+\sum a_{i} E_{i}, \quad a_{i}>0, \text { так как } X \operatorname{trm}, \\
& H_{W}=\alpha^{*} H-\sum b_{i} E_{i}, \quad b_{i} \geqslant 0, \text { так как дивизор } H \text { эффективен. }
\end{aligned}
$$

Каноничность пары $(X, c H)$ означает $a_{i}-c b_{i} \geqslant 0$. Отсюда

$$
c=c(X, H)=\min \left\{\frac{a_{i}}{b_{i}}\right\} .
$$

Обратная величина $\lambda=1 /$ с назьвается максимальной кратностью линейной системы $\mathscr{H}$ на $X$, а соответствуюшее минимуму $E_{i}$ - максимальной особенностью (часто максимальной особенностью также назьвают $\mathrm{c}_{X} E_{i}$ ). Говорят, что $\mathscr{H}$ не имеет максимальной особенности на $X$, если $\lambda \leqslant \mu$, т.е. пара $\left(X, \frac{1}{\mu} H\right) \mathrm{cn}$.

Теорема 2.7 (неравенство Нётера-Фано). В предьдущих обозначениях если $\left(X, \frac{1}{\mu} H\right)$ - слабо каноническая модель (в частности, линейная система Н на $X$ не имеет максимальной особенности), то ұ-изоморфизм.

$B$ случае, когда $S=$ pt. - точка, әто әквивалентно следующему: если $\chi-н е$ изоморфизм, то

$$
c(X, H)<\frac{1}{\mu}
$$

(т.е. $\mathscr{H}$ имеет максимальную особенность).

ДокАЗАТЕЛЬСтво. (Сp. с [8; гл. II, 1.3], [25].) По нашей конструкции и условию теоремы $1 / \mu \leqslant 1$ и пара $\left(X, \frac{1}{\mu} H\right)$ - слабо каноническая модель (cn и даже lt) пары $\left(X^{\prime}, \frac{1}{\mu} H^{\prime}\right)$. Значит, последняя - тоже не лограсслоение Мори в силу (i) предложения 2.5 , в частности, для стягивания $X^{\prime} \rightarrow S^{\prime}$. Значит, $1 / \mu \geqslant 1 / \mu^{\prime}$, так как $1 / \mu^{\prime}$ - антиканонический порог, т.е. наименьшее положительное число $a$, для которого $K_{X^{\prime}}+a H^{\prime}$ численно эффективен над $S^{\prime}$. (Однако в бирациональной геометрии чаще используется обратная величина $\mu^{\prime}=1 / a$, назьваемая квазиәффективныл порогом: в данной ситуации это такое рациональное число $\mu^{\prime}$, что $\mu^{\prime} K_{X^{\prime}}+H^{\prime} \equiv 0$ над $S^{\prime}$.) Как было отмечено вьше, пара $\left(X^{\prime}, \frac{1}{\mu^{\prime}} H^{\prime}\right)$ - слабо каноническая модель (cn) пары $\left(X, \frac{1}{\mu^{\prime}} H\right)$. Значит, по тем же соображениям $1 / \mu^{\prime} \geqslant 1 / \mu$, откуда $1 / \mu=1 / \mu^{\prime}$, или $\mu=\mu^{\prime}$.

Проверим теперь, что $\chi$ - изоморфизм в коразмерности 1 (точнее, логфлоп). Так как модель $\left(X^{\prime}, \frac{1}{\mu^{\prime}} H^{\prime}\right)$ минимальна, то, согласно доказательству (vi) предложения 2.5 , 
$\chi$ не сдувает дивизоров. По конструкции $\varphi^{\prime}=I\left(X^{\prime}, \frac{1}{\mu^{\prime}} H^{\prime}\right)$ - расслоение Иитаки, а $S^{\prime}-$ модель Иитаки пары $\left(X^{\prime}, \frac{1}{\mu^{\prime}} H^{\prime}\right)$ при достаточной обильности подкрутки $A^{\prime}$. Отсюда по “без лог” варианту (iii) предложения 2.5 преобразование $\varphi^{\prime} \circ \chi: X \rightarrow S^{\prime}$ является морфизмом (тоже расслоение Иитаки). Поскольку $\chi$ не сдувает дивизоров, то $\rho\left(X / S^{\prime}\right) \leqslant \rho\left(X^{\prime} / S^{\prime}\right)=1$. Более того, вьполнено равенство, так что $\chi^{-1}$ не сдувает дивизоров. Действительно, $\rho\left(X / S^{\prime}\right) \geqslant 1$, поскольку дивизор $H$ положительно пересекает кривые слоев $\varphi$, являющихся также кривьми над $S^{\prime}$. По проективности $X$ дивизор $H$ ө-обилен.

Итак, $\mu=\mu^{\prime}$, а пары $\left(X, \frac{1}{\mu} H\right)$ и $\left(X^{\prime}, \frac{1}{\mu} H^{\prime}\right)$ суть минимальные модели друг друга (cp. c (vi) предложения 2.5).

Закончить доказательство можно любым из двух способов: либо использовать тот факт, что $\chi$ - изоморфизм в коразмерности 1 , переводящий $\varphi^{\prime} \circ \chi$-обильный дивизор $H$ в $\varphi^{\prime}$-обильный дивизор $H^{\prime}$, либо заметить, что пары $\left(X, \frac{1}{\mu} H\right),\left(X^{\prime}, \frac{1}{\mu} H^{\prime}\right)$ терминальны в силу их минимальности. Выберем $0<\varepsilon \ll 1$, чтобы модели $\left(X^{\prime},\left(\frac{1}{\mu}+\varepsilon\right) H^{\prime}\right)$ и $\left(X,\left(\frac{1}{\mu}+\varepsilon\right) H\right)$ были каноническими. Тогда изоморфизм получаем по теореме $2.3(\mathrm{i})$.

ЛинКАЖ 2.8 (Исковских-Саркисов-Рид-Корти). Всякое бирациональное отображение между расслоениями Мори, как в диаграмме (2.4), предположительно раскладывается в конечную последовательность әлементарных модификаций, называемых линками (см. [6], [25; Chapter 13], [8; гл. II, § 1, 2]).

НАБросок конструкции. Воспользуемся предыдушими обозначениями. Для разложения модификации $\chi: X \rightarrow X^{\prime}$ фиксируем, как в (2.4), очень обильную линейную систему $\mathscr{H}^{\prime}=\left|-\mu^{\prime} K_{X^{\prime}}+\varphi^{\prime *} A^{\prime}\right|$ на $X^{\prime}$. Для произвольного расслоения Мори $\varphi: X \rightarrow S$ и его модификации $\chi$ при фиксированной паре $\left(X^{\prime}, \mathscr{H}^{\prime}\right)$ определяется $с т е-$ пень $\operatorname{deg}\left(\chi, \mathscr{H}^{\prime}\right)=(\mu, \lambda, e)$, где $\mu$-квазиэффективный порог, т.е. рациональное число такое, что $\mu K+\chi_{*}^{-1} H^{\prime}=\mu K+H \equiv 0$ над $S$ (т.е. $\left.H \sim_{\mathbb{Q}}-\mu K+\varphi^{*} A, A \in \operatorname{Div}(S) \otimes \mathbb{Q}\right)$, $\lambda=1 / c$ - максимальная кратность системы $\mathscr{H}, e-$ число максимальных особенностей. Тройки $(\mu, \lambda, e)$ упорядочены лексикографически и процесс раскручивания заключается в конструкции последовательности линков $\Phi_{1}, \Phi_{2}, \ldots$ таких, что для $\chi_{i}=\chi_{i+1} \circ \Phi_{i+1}, \chi_{0}=\chi$, степени уменьшаются: $\operatorname{deg}\left(\chi_{i+1}, \mathscr{H}^{\prime}\right)<\operatorname{deg}\left(\chi_{i}, \mathscr{H}^{\prime}\right)$. Процесс должен обрываться на изоморфизме, критерий которого доставляет неравенство Нётера-Фано.

Конструкция линков осуществляется посредством логПММ; для доказательства завершения алгоритма разложения требуются еще условия обрыва для квазиэффективных или канонических порогов (см. подробности в [25; Chapter 13], [8; гл. II, §1 2]). Возможность разложения в размерности 2 - это классический результат, восходящий к М. Нётеру и Г. Кастельнуово. В размерности 3 доказательство получено Корти [6].

2.9. География линкажа и логмоделей. Две $\mathbb{R}$-границы $B$ и $B^{\prime}$ многообразия $X$ назовем модельно эквивалентными, если все результирующие логмодели логпар $(X, B)$ и $\left(X, B^{\prime}\right)$, т.е. логминимальные модели $\left(Y, B_{Y}^{\log }\right)$ и $\left(Y, B_{Y}^{\prime \log }\right)$ или лограсслоения Мори $\left(Y, B_{Y}^{\log }\right) \rightarrow S$ и $\left(Y, B_{Y}^{\prime \log }\right) \rightarrow S$, одинаковы по первому аргументу (многообразию $Y$ ) - и имеют одинаковые структурные морфизмы. Согласно предложению 2.5 (i), а точнее по его варианту для более общих преобразований границ (ср. с рассуждением в доказательстве предложения 2.5 (iii); см. подробности в [36; 2.4.1]), 
возможны два взаимно исключаюших случая:

LMP (логминимальные пары): $\left(Y, B_{Y}^{\log }\right) \rightarrow S$ - логминимальная модель $(X, B)$ с расслоением Иитаки тогда и только тогда, когда $\left(Y, B_{Y}^{\prime \log }\right) \rightarrow S$ - логминимальная модель $\left(X, B^{\prime}\right)$ с тем же расслоением Иитаки, либо

LFM (лограсслоения Мори): $\left(Y, B_{Y}^{\log }\right) \rightarrow S-$ лограсслоение Мори тогда и только тогда, когда $\left(Y, B_{Y}^{\prime \log }\right) \rightarrow S$ - лограсслоение Мори.

Фиксируем конечное множество простых дивизоров $D_{i}$ многообразия $X$. Тогда предположительно логПММ задает разбиение единичного куба границ

$$
\sum[0,1] D_{i}
$$

на классы эквивалентных гранищ $B=\sum b_{i} D_{i}$.

ГипотезА 2.10 (о географии логмоделей). Указанное разбиение полиэдрально. Точнее, каждая его страна - класс эквивалентности - есть полиэдр, открытьй в кубе. Более того, все полиэдры выпуклы, рациональны, конечны, а разбиение локально конечно в $\sum(0,1] D_{i}$; для минимальных моделей можно включать $b_{i}=0$. При этом подполиэдр логминимальных моделей вьпукл, замкнут, конечен и рационален.

Аналогичное ожидается в категории с собственным образом гранищы (ср. с примером 2.11 ниже). При этом модели с терминальньми особенностями могут не заполнять весь куб, а лишь его подполиэдр с более грубым отношением эквивалентности.

Гипотеза установлена для логминимальных моделей в размерности 3 [36; 6.20].

ПримеР 2.11 (линкаж двух моделей). Рассмотрим два расслоения Мори $X \rightarrow S$ и $Y \rightarrow T$ с бирационально эквивалентными $X$ и $Y$. Выберем простой очень обильный дивизор $D$ на $X$, как $H^{\prime}$ в линкаже 2.8 , аналогично - $L_{Y}$ на $Y$ с собственным образом $L$ на $X$. Тогда модельная эквивалентность с собственньм образом гранищы задает разбиение квадрата $[0,1] D \oplus[0,1] L$. По конструкции вершины $D=1 D+0 L$ и $L=0 D+1 L$ имеют минимальные ${ }^{1}$ модели $(X, D)$ и $\left(Y, L_{Y}\right)$, которые также каноничны, а $0=0 D+0 L-$ два (лог)расслоения Мори. Аналогично (и даже согласно) географии логмоделей имеется вьпуклая вниз конечная цеп отрезков (эквивалентно, интервалов и точек - сепаратриса), отделяющая минимальные модели от лограсслоений Фано (овец от козлищ). Точки многоугольников разбиения, не пересекаюших гранищы квадрата по отрезку, отвечают $\mathrm{klt}$ парам $\left(W, d D_{W}+l L_{W}\right), d, l \in(0,1), \mathrm{c} D_{W}, L_{W} \neq 0$, потому эти пары сn и даже чисто trm. В частности, последнее верно для внутренних точек отрезков цепи. Соответствующие отображения Иитаки $\left(W, d D_{W}+l L_{W}\right) \rightarrow U$ - не сдутия. В противном случае малое уменьшение $d$ или $l$ снова дает минимальную и даже каноническую модель после стягивания Иитаки. (Малое уменьшение сохраняет объемность!) Более того, уменьшая $l$, по логПММ над $U$ модифицируем (обобщенными флопами $)$ пару $\left(W, d D_{W}+l L_{W}\right) \rightarrow U$ в пару $\left(W^{\prime}, d D_{W^{\prime}}+l L_{W^{\prime}}\right) \rightarrow U$, которая cn, пара $\left(W^{\prime}, d D_{W^{\prime}}\right) \operatorname{trm}$, имеет лограсслоение $\Phi$ ано $\left(W^{\prime}, d D_{W^{\prime}}\right) \rightarrow U^{\prime}$ и Мори $W^{\prime} \rightarrow U^{\prime}$

\footnotetext{
${ }^{1}$ Терминальность при этом понимается, как и логтерминальность, для некоторого разрешения; аналогично определяем чистую терминальность, которая совпадает с обычной в размерности $\geqslant 2 ;$ дивизориальную терминальность и т. п.
} 
(над $U$ ). Это верно для всех внутренних точек отрезка. Проекция на $[0,1] D$ дает линкаж от $Y \rightarrow T$ к $X \rightarrow S$. Проход через конщы отрезков отвечает линкам, возможно не элементарным. (Элементарности можно добиться, возмушая $D$ как b-дивизор.) Обрывы скрыты в порогах при определении полиэдральной структуры.

По $[36 ; 6.20]$ это - доказательство сушествования линкажа в размерности $\leqslant 3$.

\section{§. Модификации логминимальных моделей: флопы и география логмоделей}

3.1. В обычной теории Мори минимальная модель - это нормальное проективное $\mathbb{Q}$-факториальное многообразие $X$ с не хуже чем терминальньми особенностями и численно эффективным каноническим дивизором $K$. В случае поверхностей терминальных особенностей нет, т.е. trm свойство эквивалентно неособости, и, как хорошо известно, минимальная модель, если она существует, единственна в своем бирациональном классе. Для $\operatorname{dim} X \geqslant 3$ это уже не так и неизоморфных бирационально эквивалентных минимальных моделей может быть много. Однако бирациональные отображения между ними вполне обозримы: они являются изоморфизмами в коразмерности 1 и ожидается, что они разложимы в последовательности элементраных модификаций - экстремальных флопов. Подобное применимо и к логПММ. Напомним определение этого понятия.

ОПРЕДЕЛЕНИЕ 3.2. (i) Пусть $X$ - нормальное $\operatorname{trm}$ многообразие, $\varphi$ - его малое сдутие, а $D-\mathbb{R}$-Картье дивизор на $X$. Флопом многообразия $X$ или сдутия $\varphi$ назьвается малая модификация $\chi$ (над $Y$ ) в коммутативной диаграмме

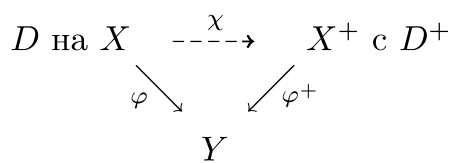

такой, что $K$ численно $\varphi$-тривиален, $\varphi^{+}{ }_{-}$тоже малое сдутие и $K_{X}+$ численно $\varphi^{+}$-тривиален. Флоп называется направленным относительно дивизора $D$ или $D$-флопом, если дивизор $-D$ численно $\varphi$-обилен, а $D^{+}=D_{X^{+}}=\chi_{*} D$ численно $\varphi^{+}$-обилен. Эквивалентно, $\chi-D$-флип как в (d) 1.1 с $\varphi_{i}=\varphi:\left(X_{i}, D_{i}\right)=(X, D) \rightarrow Y_{i}=Y$. Также флоп обычно предполагают әкстремальным, т.е. что сдутие $\varphi$ экстремально, а дивизор $D$ - эффективным.

(ii) Если в (3.1) вместо $X$ рассмотреть lt или lс логпару $(X, B)$ с границей $B$, а вместо канонических дивизоров $K$ и $K_{X}$ в взять соответственно логканонические $K+B$ и $K_{X+}+B^{+}$, где $B^{+}=\chi_{*} B$, то эта диаграмма задает (обычный) логфлоп пары $(X, B)$ или ее сдутия $\varphi:(X, B) \rightarrow Y$. При этом направленность относительно $D$ определяется как и вьше в (i).

(iii) Если в (ii) не предполагать сдутие $\varphi$ мальм и экстремальным, а потребовать лишь вьполнения следующих условий:

a) $X^{+}$нормально и (3.1) задает (обобщенный) $D$-фллип (т.е. $-D$ численно $\varphi$-обилен, $\varphi^{+}$- малое сдутие и $D^{+}=\chi_{*} D$ численно $\varphi^{+}$-обилен);

b) $K+B$ численно $\varphi$-тривиален и $K_{X+}+B^{+}$численно $\varphi^{+}$-тривиален,

то $\chi$ называется (обобщенньм) направленным логфлопом относительно $D$. 
ЗАмЕчАния 3.3. (i) Можно определить более обший логфлоп как крепантную модификацию, если в (iii) отказаться от условия, что $\varphi^{+}$является малым сдутием, а вместо этого потребовать его крепантность (см. определение 4.9 (iv)), что однозначно определит дивизор $B^{+}$, которцй по прежнему надо предполагать границей. При этом для направленного флопа дивизор $D^{+}$определяется (не однозначно) соотношением $\varphi_{*} D=\varphi_{*}^{+} D^{+}$. Такие морфизмы возможны при изучении модификаций слабо логканонических моделей и ограничении обычных логф́лопов (или логфилипов) на подмногообразия.

(ii) По определению (и при эффективности $D$ ) направленньй klt логфлоп и тем более (терминальный) флоп являются логфлиипами относительно $K+B+\varepsilon D, 0<\varepsilon \ll 1$, в определении (ii) и (iii) и $K+\varepsilon D$ в (i). Поэтому вопрос о существовании и обрыве таких логфилопов и флопов является частным случаем обших гипотез о существовании и обрьве логфлипов. Гипотеза о существовании в этом общем контексте доказана в размерности 3 и 4 , обрьв - в размерности 3 и для терминального случая в размерности 4 (см. [34; Theorem], [36; 5.2], [37; 1.8, 1.9], [17], [12], [39; Example 9]). Однако первые доказательства существования терминальных флопов в размерности 3 (и некоторых в размерности 4) были непосредственными, использующими классификацию 3 -мерных терминальных особенностей (теорему Г. Н. Тюриной о разрешении дювалевской 2-мерной особенности в 1-мерном семействе) (см. [23; 2.2], [11; 4.1]). Обрьв таких флопов, согласно указанной идее - сведения к флипам, в размерности 3 устанавливается довольно легко (см. [33; 2.17], [20], [25]). Мы воспроизводим здесь доказательство в целях иллюстрации простейшего подхода к такого типа проблемам. Общий подход обсуждается в [39].

Основньм ингредиентом здесь и во многих других вопросах теории (лог) минимальных моделей является следуюшая лемма.

Лемма $3.4[33 ; 2.13 .3,2.15]$. (i) Пусть $(X, D)-\rightarrow\left(X^{+}, D^{+}\right)-$логфлип сдутия $\varphi: X \rightarrow Y$ в сдутие $\varphi^{+}: X^{+} \rightarrow Y$ как в диаграмме (3.1) (см. также доказательство). Тогда для любого простого b-дивизора $E$ многообразия $X$ (равносильно, многообразия $X^{+}$)

$$
a\left(E, X^{+}, D^{+}\right) \geqslant a(E, X, D) .
$$

Более того, неравенство строгое тогда и только тогда, когда $\mathrm{c}_{X} E \subset \operatorname{Exc}(\varphi)$ (әквивалентно, $\mathrm{c}_{X^{+}} E \subset \operatorname{Exc}\left(\varphi^{+}\right)$).

(ii) Пусть $(X, B) \operatorname{trm}, \operatorname{dim} X=3$ и $B-\mathbb{R}$-граница. Тогда существует конечное число простых $b$-дивизоров $E_{1}, \ldots, E_{s}$ таких, что если $а(E, X, B)<1$ для исключительного на $X$ простого b-дивизора $E$, то $E$ - либо одно из $E_{i}, i=1, \ldots, s$, либо $E$ отвечает раздутию общей точки кривой $\mathrm{c}_{X} E$, лежащей в неособой час$m u \operatorname{Supp} B, B=\sum b_{j} D_{j}$, и автоматически вне терминальных (следовательно, изолированных) особенностей $X$. Более того, в последнем случае

$$
a(E, X, B)=1-b_{j},
$$

әде $j$ - номер компоненты $D_{j} c \mathrm{c}_{X} E \subset D_{j}$. 
ДокАЗАТЕльство. Утверждение (i) хорошо известно, начиная с [33], и следует непосредственно из более обшей леммы 2.4. Поэтому и логфлип можно полагать более обшим, требуя лиш численную $\varphi$-обильность $-(K+D)$ и численную $\varphi^{+}$-обильность $K_{X^{+}}+D^{+}$, при этом дивизор $D$ не обязательно эффективен, а сдутия $\varphi$ и $\varphi^{+}-$экстремальны.

Докажем (ii). Пусть $f: V \rightarrow X$ - логразрешение пары $(X, B)$, на котором неприводимые компоненты $\operatorname{Supp} B_{V}$ попарно не пересекаются. Имеем $K_{V}=f^{*}(K+B)+$ $\sum a_{i} E_{i}$, где $a_{i}>0$ для всех исключительных дивизоров сдутия $f$, поскольку $X$ предполагается trm. Любой исключительньй на $X$ простой b-дивизор ${ }^{2} E$, отличный от построенных $E_{i}$, может быть получен последовательностью раздутий с неособыми центрами, начиная с $V$. Так что если $a(E, X, B)<1$, то $E$ либо отождествляется с одним из $E_{i}$, либо отвечает раздутию общей точки кривой с ${ }_{X} E$, лежашей в неособой части $\operatorname{Supp} B$.

Теорема 3.5 (обрьв терминальных флипов и флопов в размерности 3 ). (i) Последовательность 3-мерных логфлипов с исходной $\operatorname{trm}($ и даже $\mathrm{cn})$ логпарой $(X, B)$, где $B-\mathbb{R}$-граница, обрыввется.

(ii) Последовательность 3-мерных (терминальных) направленных D-флопов

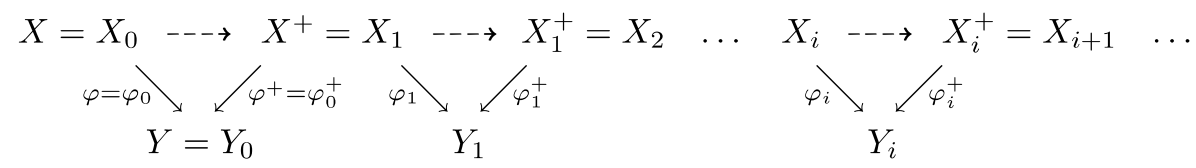

где $D$ - эффективныи $\mathbb{R}-$ Картье дивизор и $X \mathrm{trm}$, обрывается.

ДокАЗАТЕльство. (Cp. с [39; Example 9].) Для любого достаточно малого положительного $\varepsilon \mathbb{R}$-дивизор $B=\varepsilon D$ является гранищей и логпара $(X, B) \operatorname{trm}$. Тогда все $D$-фллопы суть логфлипы пары $(X, B)$ с малыми сдутиями $\varphi_{i}$ и $\varphi_{i}^{+}$. (Флопы малы, т.е. являются малыми модификациями. В частности, они не сдувают компонент границы. Значит, все модификации $\left(X_{i}, B_{X_{i}}\right) \operatorname{trm}$ и $X_{i} \operatorname{trm}$ по эффективности $B_{X_{i}}$.) Поэтому достаточно установить обрыв логфлипов. Собственные образы $B$ будем обозначать той же буквой $B$ на всех модификациях $X_{i}$.

Пусть $B=\sum b_{i} D_{i}$. Упорядочим все ненулевые значения кратностей границы: $b_{1}>b_{2}>\cdots>b_{s}$, а также добавим $b_{0}=1$ и $b_{s+1}=0$.

По лемме 3.4 и так как перестроенное исключительное множество $\operatorname{Exc}\left(\varphi_{i}\right)$ содержит кривую, то все минимальные дискрепантности $\operatorname{dis}\left(\varphi_{i}\right)<1$, где

$$
\operatorname{dis}\left(\varphi_{i}\right)=\min _{E}\left\{a\left(E, X_{i}, B\right) \mid \mathrm{c}_{X_{i}} E \subset \operatorname{Exc}\left(\varphi_{i}\right)\right\}
$$

Значит, с точностью до конечного числа логфлипов для некоторого целого числа $0 \leqslant j \leqslant s$ можно предполагать, что минимальная дискрепантность $\operatorname{dis}\left(\varphi_{i}\right) \geqslant 1-b_{j}$ для всех сдутий $\varphi_{i}$ и для бесконечного их числа $\operatorname{dis}\left(\varphi_{i}\right)<1-b_{j+1}$. Обрьв устанавливается согласно обшим принципам (см. [39]), но из-за недоказанности некоторых обших гипотез о поведении дискрепантностей приходится прибегать к специальным трюкам. В частности, в нашем случае мы заменяем минимум по всем

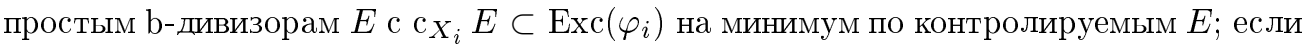

\footnotetext{
${ }^{2} \mathrm{Cм.} \mathrm{определение} 5.5$ ниже.
} 
таковых нет, то индекс $j$ полагаем равным $s$, а контролируемую дискрепантность $\varphi_{i}$ равной $1-b_{s}$. По определению $E$ контролируем, если $c_{X_{i}} E-$ кривая в $\operatorname{Exc}\left(\varphi_{i-1}^{+}\right) \cup \operatorname{Exc}\left(\varphi_{i}\right)$ для бесконечного числа модификаций. В конце конщов мы установим отсутствие таких $E$. C другой стороны, контролируемые $E$ существуют по предположению бесконечности последовательности логфлипов, что и дает обрьв.

Сначалапроверим, что для почти всех (кроме конечного числа) неконтролируемых b-дивизоров $E$ многообразия $X$, лежаших над $\operatorname{Exc}\left(\varphi_{i}\right)\left(\right.$ или $\left.\operatorname{Exc}\left(\varphi_{i}^{+}\right)\right)$при некоторой модификации $X_{i} \rightarrow X_{i+1}$,

$$
a(E, X, B) \geqslant 1-b_{j+1}
$$

Действительно, в противном случае по лемме 3.4 имеется бесконечное число неконтролируемых $E$ многообразия $X$, исключительных на $X$ по малости модификаций и лежащих над некоторым исключительным множеством $\operatorname{Exc}\left(\varphi_{i}\right)\left(\right.$ или $\left.\operatorname{Exc}\left(\varphi_{i}^{+}\right)\right)$, с

$$
a(E, X, B) \leqslant 1-b_{l}, \quad l \leqslant j
$$

и равенство $a(E, X, B)=1-b_{l}$ достигается на бесконечном числе b-дивизоров $E$ с кривой $\mathrm{c}_{X} E$. Эти кривые лежат на конечном числе простых дивизоров $D_{j}$ в $X$ с кратностями гранищы $b_{l}$. Также можно предполагать $l$ наименшшим с данньми кривыми. Тогда с точностью до конечного числа логфлипов все оставшиеся сдувают указанные кривые $c_{X} E$ на этих дивизорах, не раздувая новых кривых по лемме 3.4 и выбору контролирумого $b_{j}$, что невозможно по ограниченности числа Пикара данных дивизоров (ср. со случаем I ниже).

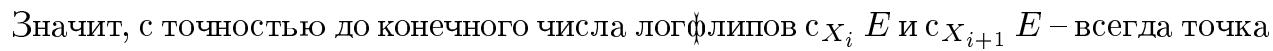
для всякого неконтролируемого $E$, лежащего над $\operatorname{Exc}\left(\varphi_{i}\right)$ и $\operatorname{Exc}\left(\varphi_{i}^{+}\right)$, с $a\left(E, X_{i}, B\right)<$ $1-b_{j+1}$. Таким образом, при бесконечности цепочки логфлипов для всякого сдутия

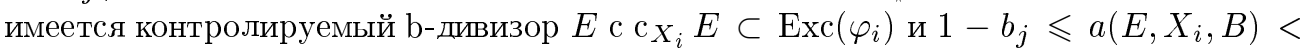
$1-b_{j+1} \leqslant 1$. В противном случае $j=s$. Но тогда по лемме $3.4(\mathrm{i})$ раздутие над

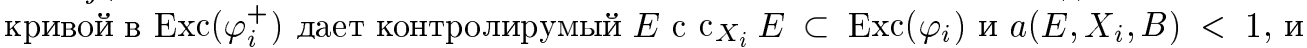
контролируемая минимальная дискрепантность $\operatorname{dis}\left(\varphi_{i}\right)<1$ определена.

Случай I. Имеется бесконечное число сдутий $\varphi_{i} \mathrm{c} \operatorname{dis}\left(\varphi_{i}\right)=1-b_{j}$. Можно также считать, что для бесконечного числа $\varphi_{i}$ минимум дискрепантностей достигается в b-дивизоре $E$ с центром с ${ }_{X_{i}} E$ - кривой. В противном случае c $_{X_{i}} E=$ pt. - точка для бесконечного числа $\varphi_{i}$. Согласно лемме 3.4 (ii), все точки $\mathrm{c}_{X_{i}} E$ для контролируемых $E$ с $a\left(E, X_{i}, B\right)=1-b_{j}$ образуют 0-мерное подмногообразие $W_{i} \subset X_{i}$. Теперь, согласно лемме 3.4 (i) и нашим условиям, новые такие точки не появляются на следующем $X_{i+1}-$ модификации с $\operatorname{dis}\left(\varphi_{i}\right)>1-b_{j}$ не задевают $W_{i}$, а при модификациях с $\operatorname{dis}\left(\varphi_{i}\right)=1-b_{j}$ по крайней мере одна точка $W_{i}$ исчезает. Это дает требуемую бесконечность с центром с $_{X_{i}} E$ - кривой.

Снова по лемме 3.4 (ii) имеется собственное подмногообразие $W_{i} \subset X_{i}$ (возможно с недивизориальньми компонентами), общие кривые компонент которого суть $\mathrm{c}_{X_{i}} E$ для b-дивизоров $E$ с $a\left(E, X_{i}, B\right)=1-b_{j}$. Дивизориальные компоненты (поверхности) $W_{i}$ суть в точности компонентыграницы $B$ с кратностями $b_{j}$. Нетрудно проверить, что обычная минимальная дискрепанта $\leqslant 1-b_{j}$ над общей точкой любой кривой в $W_{i}$. Опять согласно лемме 3.4 (i) и нашим предположениям, модификации не раздувают 
кривых в $W_{i}$, но лишш сдувают, когда $\operatorname{dis}\left(\varphi_{i}\right)=1-b_{j}$ и равенство достигается на $E$ с $\mathrm{c}_{X_{i}} E-$ кривой. Значит, этот случай невозможен.

Случай II. С точностью до конечного числа логфлипов $\operatorname{dis}\left(\varphi_{i}\right)>1-b_{j}$ для всех сдутий $\varphi_{i}$. По лемме 3.4 (ii) значения $\operatorname{dis}\left(\varphi_{i}\right)<1-b_{j+1}$ достигаются на конечном множестве контролируемых $E$. По контролируемости $a\left(E, X_{i}, B\right)$ удовлетворяют обрьву возрастающих цепей и стабилизируются. Поэтому значение $a=\operatorname{dis}\left(\varphi_{i}\right)>b_{j}$ тоже стабизизируется, т.е. все $\operatorname{dis}\left(\varphi_{i}\right) \geqslant a$ и равенство достигается для бесконечного числа $\varphi_{i}$. Те же рассуждения, что в случае I, приводят к обрьву, т.е. к отсутствию контролируемых $E$. При этом $W_{i}$ - конечное объединение кривых и точек.

3.6. Приложим теперь географию логмоделей к вопросам о модификации минимальных (логминимальных) моделей из одного бирационального класса. Для несобых поверхностей, как известно, минимальная модель единственна в своем бирациональном классе. Более того, оказывается, что единственна также 2-мерная минимальная (или терминальная) модель логпары $(X, B)$, поскольку такие модели изоморфны в коразмерности 1 (см. предложение 2.5 (vi)).

Однако, это уже не так в размерности 3 и вьше: сушествует, вообше говоря, много минимальных (соответственно логминимальных) моделей в фиксированном бирациональном классе (соответственно логпары или морфизма логпары). Они связаны между собою следуюшим образом (см. предложение 2.5 (vi) выше, предложение 3.10 и теорему 3.11 ниже): любые две минимальные (соответственно логминимальные) модели в одном бирациональном классе (соответственно модели одного типа логпары или морфизма логпары) изоморфны в коразмерности 1 и получаются одна из другой последовательностью флопов (соответственно логфлопов) в строгом случае. Более глобальное описание всех минимальных моделей в бирациональном классе можно дать в терминах географии логмоделей (см. гипотезу 2.10 выше).

ОПРЕДЕЛЕНИЕ 3.7. Логминимальнье модели (проективно) одного типа, когда имеют общее (проективное) логразрешение и являются его логминимальными моделями. В качестве границы на разрешении берется логобраз.

ЗАмЕчАниЕ 3.8. Существование обшего (и даже проективного) логразрешения в последнем определении вьполнено всегда для логмоделей, имеюших лиш klt и plt, в частности, trm особенности.

Из логПММ связности логминимальных моделей следует, что они проективно одного типа. Обратное верно по модулю гипотез о существовании и обрьва флипов. Напомним, что две логминимальные модели логПММ связны, если они получаются в результате логПММ из одной строго lt логпары. Отметим также, что логфлипы сохраняют проективность моделей и dlt особенностей, т.е. lс центры логнеособы [34; c. 109]. По лемме 2.4, точнее по независимости дискрепантностей от слабо логканонической модели (см. доказательство предложения 2.5 (iii)) и лемме 3.4 (i) эти центры совпадают на таких логминимальных моделях, а потому имеется общее логразрешение.

Пример 3.9 (перестройка Куликова). Это экстремальный флоп (и логфлоп) логнеособой логпары $\left(X_{1}, B_{1}\right)$ в $\left(X_{2}, B_{2}\right)$, где $B_{1}=B_{2}=\sum_{i=1}^{4} D_{i}$ в бирациональном смысле, а кривую $C_{1}=D_{1} \cap D_{3} \subset X_{1}$ флоп преобразует в $C_{2}=D_{2} \cap D_{4} \subset X_{2}$. 
Логпары $\left(X_{1}, B_{1}\right)$ и $\left(X_{2}, B_{2}\right)$ не имеют одного типа, но являются логминимальными моделями друг друга.

ПРЕДЛОЖЕНИЕ 3.10. (i) Любъе две логминимальнье модели $\left(X_{1}, B_{1}\right) u\left(X_{2}, B_{2}\right)$ друг друга изоморфны в коразмерности 1.

(ii) Любье две логминимальнье модели $\left(X_{1}, B_{1}\right)$ u $\left(X_{2}, B_{2}\right)$ одного типа изоморфны в коразмерности 1. При этом логразрешения в определении одного типа можно брать для каждой модели свое, но тогда разрешения предполагают (лог) изоморфньми в коразмерности 1.

ДокАЗАТЕЛЬСтвО. Утверждение (i) следует непосредственно из определения, и оно проще, чем доказательство предложения 2.5 (vi).

Утверждение (ii) по существу сводится к (i). Пусть $\left(Y_{1}, B_{1}^{\log }\right)$ и $\left(Y_{2}, B_{2}^{\log }\right)$ - логразрешения пар $\left(X_{1}, B_{1}\right)$ и $\left(X_{2}, B_{2}\right)$ в определении одного типа, изоморфные в коразмерности 1. Пусть $D$ - простой дивизор на $X_{1}$, исключительньй на $X_{2}$. Тогда он является дивизором на $Y_{2}$. По определению логтерминального разрешения и одного типа $a\left(D, X_{2}, B_{2}\right)>a\left(D, Y_{2}, B_{2}^{\log }\right)=a\left(D, Y_{1}, B_{1}^{\log }\right)=a\left(D, X_{1}, B_{1}\right)=-b$, т.е. $\left(X_{2}, B_{2}\right)-$ логминимальная модель пары $\left(X_{1}, B_{1}\right)$. Также верно обратное.

УСЛОВНАЯ ТЕОРЕМА 3.11 (по модулю гипотез о сушествовании и обрыве направленных флопов в (i) и логфлопов в (ii)). (i) Проективные минимальные модели в одном бирациональном классе связаны последовательностью әкстремальных флопов и обратных к ним.

(ii) Проективнье логминимальные ( и минимальные) модели (логпары $(X, B)$ ) проективно одного типа связаны последовательностью әкстремальных логфлопов и обратных к ним (см. доказательство).

Флопь и логфлопы строго минимальных моделей (соответственно строго логминимальных моделей одного типа) можнно предполагать направленными и экстремальным.ми.

ДокАЗАТЕльство. Будем доказьвать только утверждение (ii) ((i) доказывается аналогично).

Пусть $\left(X_{1}, B_{1}\right), B_{1}=B_{X_{1}}^{\log }$, и $\left(X_{2}, B_{2}\right), B_{2}=B_{X_{2}}^{\log }$,- две проективные модели пары $(X, B)$ одного типа. Логпару $(X, B)$ можно предполагать общим проективньм логразрешением. По проективности на $X_{2}$ имеется обильньй эффективньй $\mathbb{Q}$-дивизор $D$ такой, что $\left(X_{2}, B_{2}+\varepsilon D\right)$ - логминимальная и в то же время логканоническая модель пары $\left(X, B+\varepsilon D_{X}\right)$ при любом $0<\varepsilon \ll 1$. По одинаковости типов $\left(X_{1}, B_{1}+\varepsilon D_{X_{1}}\right)$ lt при любом $0<\varepsilon \ll 1$ и с точностью до численной эффективности - модель того же типа при $\mathbb{Q}$-факториальности $X_{1}$. Последнего можно добиться после $\mathbb{Q}$-факториализации (см. следствие 6.7 ниже), что снова сохраняет тип; при проективной $\mathbb{Q}$-факториализации получаем строго логминимальную модель. Пара $\left(X_{1}, B_{1}+\varepsilon D_{X_{1}}\right)$, однако, не обязательно логминимальная модель пары $\left(X, B+\varepsilon D_{X}\right)$ : дивизор $K_{X_{1}}+B_{1}+\varepsilon D_{X_{1}}$ не обязательно численно эффективен. Чтобы добиться численной эффективности, применяем логПММ. Наконец, обрыв и сдутие на логканоническую модель дает $X_{2}$ по теореме 2.3 о единственности. С дутие сушествует для объемного $D$. С помощью географии логмоделей $\mathbb{Q}$-факториализацию можно разложить в композицию обратных к экстремальным логф лопам, а логканоническое сдутие - в композицию экстремальных 
логфлопов [36; 6.22]; при этом для экстремальньх логфлопов можно дополнительно предполагать, что $\rho\left(X^{+} / Y\right) \leqslant 1$ (и возможно $\left.=0\right)$.

В строгом случае по $\mathbb{Q}$-факториальности $\mathbb{Q}$-факториализация и логканоническое сдутие не нужны, и все логфлипы пары $\left(X_{1}, B_{1}+\varepsilon D_{X_{1}}\right)$ в конструкции суть направленные экстремальные лог- $\varepsilon D_{X_{1}}$-флопы пары $\left(X_{1}, B_{1}\right)$.

Во всех конструкциях достаточно существования и обрьва направленных логфлопов (см. замечание 3.3 (ii)). В не $\mathbb{Q}$-факториальном случае они понимаются в несколько более общем смысле как в $[37 ; 1.9]$.

УСЛОВНАЯ ТЕОРЕМА 3.12 (о конечности числа минимальных моделей общего типа по модулю сушествования и обрыва направленных логфлопов [18], [20]; см. также $[36 ; 6.22],[25 ; 12.3])$. Для логпары $(X, B)$ общего типа, т.е. с обгемным $K_{Y}+B_{Y}$ для любой ее сп модификации $\left(Y, B_{Y}\right)$, имеется только конечное число проективных минимальных моделей.

ДокАЗАТЕльство. Следуем доказательству [36; 6.22]. Фиксируем простые компоненты границы $B$ и добавим конечное множество простых подвижных дивизоров

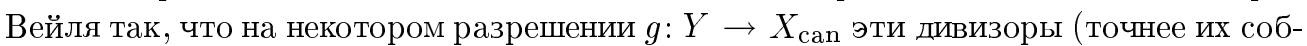
ственные образы) и исключительные дивизоры для сдутия $g$ порождают все дивизоры $Y$ по модулю численной эквивалентности. (Напомним, что $X_{\text {can }}$ обозначает каноническую модель пары $(X, B)$, которая существует для пар общего типа, т.е. $K_{Y}+B_{Y}$ объемен.) Обозначим образы этих простых дивизоров на $X_{\text {сап }}$ через $D_{i}$ и отождествим их с собственными прообразами на всех других моделях. Добавляя к ним конечное множество простых дивизоров, можно предполагать, что $H \geqslant D_{i}$ для всех $i$, где $H-$ обильный эффективный дивизор на $X_{\text {can }}$ с носителем в дивизорах $D_{i}$, проходящий через все особенности $X_{\text {can }}$.

Утверждается, что на любой минимальной модели $\left(Y, B_{Y}\right)$ можно составить эффективньй численно обильный дивизор $D=\sum d_{i} D_{i}+\sum e_{i} E_{i}$, где $E_{i}$ - исключительные на $X_{\text {сап }}$ дивизоры. Действительно, по конструкции $D_{i}$ и $E_{i}$ порождают все $\mathbb{R}$-Картье дивизоры на $Y$ с точностью до численной эквивалентности. Тем самьм, можно построить $D$ с $d_{i}, e_{i} \in \mathbb{R}$. Добавляя кратность $g^{*} H$, получаем эффективность $D$. Значит, $\left(Y, B_{Y}+\varepsilon D\right)$ - логминимальная и в то же время логканоническая модель пары $\left(X, B+\varepsilon D_{X}\right)$ при любом $0<\varepsilon \ll 1$, а потому единственная такая минимальная модель. Тем самым получается инъекция минимальных моделей в страны с носителями границ в дивизорах $D_{i}$ и исключительных на $X_{\text {can }}$ дивизорах (последних конечное число как b-дивизоров для всех минимальных моделей). Но стран в этой географии конечное число (см. [36;6.20]). В общем случае география требует логПММ для $\mathbb{R}$-дивизоров. Однако в данной ситуации достаточно существования и обрыва направленных логфлопов (ср. с замечанием 3.3 (ii)).

ЗАмечАНИЕ 3.13 (Батырев, [2]). Другое объяснение конечности в теореме 3.12 связано с конечньм полиэдральным разложением конуса эффективных с точностью до $\mathbb{R}$-эквивалентности $\mathbb{R}$-дивизоров $D$ многообразия Фано с терминальньми особенностями на подконусы поляризации проективных многообразий, полученных в результате $D$-ПММ исходя из данного многообразия (см. $[2 ; 3.4][37 ; 3.33])$. Сами дивизоры можно рассматривать по модулю численной, $\mathbb{R}$-линейной и даже просто тождественной эквивалентности, поскольку группа Пикара многообразия Фано конечно порождена. 
Это обобщается на случай klt слабых лограсслоений Фано (с возможно 0-мерными слоями), где "слабо" означает, что относительная обильность $-(K+B)$ заменяется на численную эффективность и объемность. Для доказательства теоремы 3.12 достаточен другой крайний случай, когда $\left(Y, B_{Y}\right) \rightarrow X_{\text {can }}-$ сдутие (строго) минимальной модели на каноническую.

КомментАРИй 3.14. Вопрос о количестве проективных минимальных моделей в бирациональном классе в обшем случае остается пока открытьм. Однако здесь возникает и другой вопрос - как считать количество таких моделей?

Первый способ - количество стран в географии в образе при инъекции минимальных моделей или число конусов поляризации в разложении конуса эффективных дивизоров. Это в точности множество минимальных моделей, получаемых из достаточно раздутого неособого проективного многообразия посредством ПММ. Точнее, любые две минимальные модели $X_{1}$ и $X_{2}$ в одном классе по определению бирационально изоморфны. При этом они отождествляются (рассматриваются как одна модель), если данная модификация $X_{1} \rightarrow X_{2}$ является изоморфизмом, т.е. бирегулярна.

Однако возможны и другие изоморфизмы $X_{1} \cong X_{2}$, не индуцированные бирациональными отображениями. Это приводит ко второму способу подсчета числа минимальных моделей. Ясно, что число моделей при первом подсчете не менее, чем при втором.

Единственность минимальных моделей в размерности 2 и конечность их для полных поверхностей общего типа устанавливается первым способом.

Для 3-мерных многообразий не общего типа М. Рид привел пример, когда число минимальных моделей, подсчитанных первьм способом, бесконечно. Однако не ясно даже в этом примере (см. ниже), является ли оно бесконечным при отождествлениях с точностью до произвольного изоморфизма.

Примеры 3.15. 1) [31] Пусть $f: X \rightarrow \mathbb{A}^{2}$ - семейство эллиптических кривых, заданное уравнением

$$
z_{1}^{2}=\left(\left(z_{2}-a\right)^{2}-x\right)\left(\left(z_{2}-b\right)^{2}-y\right), \quad a \neq b,
$$

где $x, y$ - координаты на $\mathbb{A}^{2}$, a $z_{1}, z_{2}$ - координаты в слоях. Слой $f^{-1} p$ является неособой эллиптической кривой, если $p \in\{x y \neq 0\}$, рациональной кривой с одной обыкновенной двойной точкой, если $p \in\{x y=0\}, p \neq(0,0)$, и парой рациональных кривых, пересекаюшихся трансверсально в двух точках, если $p=(0,0)$. Мы будем рассматривать это семейство как аналитический росток в окрестности центрального приводимого слоя. Каждую из неприводимых компонент можно последовательно флопировать, так что в результате получается счетное число минимальных моделей (конусов поляризации) над ростком $p \in \mathbb{A}^{2}$.

Но все эти минимальные модели, очевидно, изоморфны над ростком точки $p \in \mathbb{A}^{2}$, так что существует только одна минимальная модель, если считать вторым способом.

Конечно, эту локальную ситуацию можно глобализовать, взяв подходящее проективное замыкание (разрешая особенности, если необходимо).

2) Непроективных минимальных моделей может быть бесконечно много (счетное количество) даже при втором способе подсчета. Действительно, пусть $X-3$-мерная достаточно общая квинтика, тогда на ней имеются гладкие рациональные кривые $C$ 
любой натуральной степени $d$ с нормальным пучком $\mathscr{O}_{C}(-1) \oplus \mathscr{O}_{C}(-1)$. Каждая такая кривая аналитически сдувается, и флоп в ней $X \rightarrow X_{d}$ приводит к аналитическому многообразию (или алгебраическому пространству), которое не проективно и более того - не является алгебраическим многообразием. Модели $X_{d}$ попарно неизоморфны для разных $d$, поскольку $d$ - наименьший индекс пересечения кривой $C_{d} \subset X$, перестроенной флопом из $C$, с дивизорами $D<0$ на $X_{d}: D C_{d} \geqslant d$.

Во всяком случае, высказывается следующее предположение.

ГипотезА 3.16 (Кавамата, Матсуки, [25; 12-3-6]). Число проективных минимальных моделей в фиксированном бирациональном классе всегда конечно, если их рассматривать с точностью до произвольного изоморфизма.

Кавамата показал в [16], что эта гипотеза верна для моделей 3-мерных многообразий $X$ с $\kappa(X)>0$.

\section{Глава II.}

\section{3-мерный логфлип}

В этой главе мы приведем полное доказательство существования 3-мерного логтерминального по Кавамате (klt) логфлипа, следуя идее второго автора [37] (см. также [10]). В комментариях мы кратко очертим другие, более ранние, подходы к решению этой проблемы [26], [11], [34], [21]. В подготовительном $\S 4$ воспроизведена редукщия исходной задачи к проблеме сушествования допредельных флипов и специальному обрыву. В $\S 5$ сушествование допредельного флипа, как вопрос о конечно порожденности некоторой функциональной алгебры, сводится к аналогичному вопросу в размерности 2. Этот индуктивньй шаг - одна из основных идей в [37] обшего подхода к решению проблемы сушествования $n$-мерного логфлипа. Другая основная идея в [37]редукция проблемы конечной порожденности функциональных алгебр к CCS-гипотезе - излагается в $\S 6$ на примере 2-мерного случая, где эта CCS-гипотеза успешно и довольно просто доказьвается в более точной форме (см. следствие 6.6 ниже), что приводит к требуемому результату в размерности 3 . В $\S 7$ мы проверим полустабильность 3-мерного флипа.

\section{§ 4. Редукция к допредельным флипам и специальный обрыв}

ОПРЕДЕЛЕНИЕ 4.1. Пусть $(X, B)$ - klt пара и $\varphi: X \rightarrow Y$ - сдутие нормального $\mathbb{Q}$-факториального многообразия $X$ на нормальное многообразие $Y$ со следуюшими свойствами:

(i) $\varphi$ - малое сдутие, т.е. $\operatorname{codim} \operatorname{Exc}(\varphi) \geqslant 2$;

(ii) $-(K+B)$ численно $\varphi$-обилен;

(iii) $\rho(X / Y)=1$, где $\rho(X / Y)=\rho(X)-\rho(Y)$ - относительное число Пикара.

Такое сдутие назьвается klt логсдутием.

klt логфлипом сдутия $\varphi$ назьвают модификацию

$$
(X, B) \stackrel{\chi}{\varphi} \underset{\swarrow^{--\varphi^{+}}}{\longrightarrow}\left(X^{+}, B^{+}\right)
$$


где $\varphi^{+}: X^{+} \rightarrow Y-$ сдутие, для которого многообразие $X^{+}$нормально и $\mathbb{Q}$-факториально, логпара $\left(X^{+}, B^{+}\right) \mathrm{klt}$ и вьполнены следуюшие условия:

$\left(\mathrm{i}^{+}\right) \varphi^{+}$мало, т.е. $\operatorname{codim} \operatorname{Exc}\left(\varphi^{+}\right) \geqslant 2$;

(ii $\left.{ }^{+}\right) K_{X+}+B^{+}$численно $\varphi^{+}$-обилен;

$\left(\mathrm{iii}^{+}\right) \rho\left(X^{+} / Y\right)=1$;

где $B^{+}=\chi_{*} B-$ собственньй образ $B$ и $X \backslash \operatorname{Exc}(\varphi) \stackrel{\sim}{\rightarrow} X^{+} \backslash \operatorname{Exc}\left(\varphi^{+}\right)$.

КомМЕНтАРИЙ 4.2. Проблемы существования klt логфлипов и обрыва их последовательности являются основными в логПММ для категории $\mathbb{Q}$-фракториальных проективных lt многообразий. Из решения этих проблем в логПММ c klt особенностями следует, конечно, и решение аналогичных проблем в категории Мори $\mathbb{Q}$-факториальных проективных trm многообразий: достаточно положить $B=0$ и заметить, что при любых логфлипах особенности не ухудшаются, а в перестраиваемом множестве даже улучшаются (см. лемму 3.4 (i) в главе I). То же самое верно в отношении не $\mathbb{Q}$-факториальных многообразий и/или lt особенностей. По тем же соображениям в определении логфлипа достаточно потребовать нормальность $X^{+}$и условия $\left(\mathrm{i}^{+}\right),\left(\mathrm{ii}^{+}\right)$: остальные автоматически получаются из условий на $(X, B)$ и $\varphi$.

Хронологически первым было доказательство обрьва последовательности филипов в категории 3 -мерных терминальных многообразий $[33 ; 2.17]$. Существование флипов в этом случае было получено Мори [26]. Оно основано на редукции Каваматы [11] к случаю 3-мерных флопов (через двойные накрытия и гипотезу о слоне - существование хорошего эффективного дивизора в $|-K|$ или $|-2 K|)$.

Первое доказательство существования 3-мерных klt логфлипов было установлено вторым автором [34]. Для этого он ввел и использовал следующие понятия.

(i) Cпециальньй флип-это логфлип $\mathbb{Q}$-факториальной lt логпары $(X, B)$ с мальм сдутием $f:(X, B) \rightarrow Y$, где $B=S=\sum S_{i} \neq 0$ - приведенный дивизор Вейля, $-(K+S)$ и все $-S_{i} f$-обильны.

(ii) Специальный обрыв - это обрыв последовательности логфилипов

$$
\left(X_{1}, B_{1}\right) \rightarrow\left(X_{2}, B_{2}\right) \rightarrow \cdots \cdots\left(X_{i}, B_{i}\right)-\rightarrow \cdots
$$

где $\left(X_{i}, B_{i}\right)$ - lс логпары, в следующем смысле: существует $i_{0} \in \mathbb{N}$ (зависящее от последовательности) такое, что для каждого $i \geqslant i_{0}$ перестраиваемое подмножество $\operatorname{Exc}\left(\varphi_{i}\right)$ не пересекает приведенную часть границы $\left\lfloor B_{i}\right\rfloor$ (см. определение 4.6 ниже).

(iii) Условие обрыва возрастаюших цепочек (о.в.ц.) для $\bar{S}_{d}^{0}$ (local). Множество $\bar{S}_{d}^{0}($ local $)$ состоит из последовательностей $\left(b_{1}, \ldots, b_{m}\right)$ таких, что существуют $\mathbb{Q}$-факториальное многообразие $X, \operatorname{dim} X \leqslant d$, подмножество $Z \subset X$ и $\mathbb{R}$-гранища $B=B_{0}+$ $\sum b_{i} D_{i}$ с приведенной (но возможно приводимой) компонентой $B_{0} \neq 0$ такие, что $\operatorname{Supp} D_{i} \cap Z \neq \varnothing$ для всех $i, Z \subset \operatorname{Supp} B_{0}, K+B_{0}$ plt и $K+B$ максимально lс вблизи $Z$, т.е. lс и ни одну из кратностей $b_{i}$ нельзя увеличить, чтобы не нарушить логканоничности. Порядок определяется следуюшим образом:

$$
\left(b_{1}, \ldots, b_{m}\right) \leqslant\left(b_{1}^{\prime}, \ldots, b_{m^{\prime}}^{\prime}\right) \text {, если } m^{\prime}<m, \text { либо } m^{\prime}=m \text { и } b_{i} \leqslant b_{i}^{\prime} \text { при всех } i .
$$

Основньм из результатов в [34] является теорема редукции 6.4 (см. также [21; Section 18]): если (i)-(iii) вьполнены в размерности 3 (или общее $n$ ), то klt логфлипы 
сушествуют в этой размерности. Там же доказано [34; 4.1] (см. также [21; 7.1]), что специальный обрыв вьполнен в размерности 3 (как мы покажем ниже в теореме 4.8, в любой размерности $n$ это вытекает из логПММ в размерности $n-1)$. Условие (iii) (о.в.ц.) также выполнено в размерности $\leqslant 3$ (см. [34; Chicago lemma 4.9], а также [21; 18.19, 18.25.1]).

Для доказательства существования специального флипа вводится понятие дополнения.

(iv) (См. $[34 ; \S 5]$ или [21; Section 19].) Пусть $X$ - нормальное многообразие и $B=$ $\sum b_{i} D_{i}-$ субгранища (т.е. все $b_{i} \leqslant 1$, но возможны и отрицательные значения), $S-$ наименьший приведенный дивизор Вейля на $X$ такой, что $\lfloor B-S\rfloor \leqslant 0$, и пусть $B_{0}:=$ $B-S: n$-дополнением для $K+B$ называется дивизор $\bar{B} \in\left|-n K-n S-\left\lfloor(n+1) B_{0}\right\rfloor\right|$ такой, что $K+B^{+}$lс, где $B^{+}:=S+\frac{1}{n}\left(\left\lfloor(n+1) B_{0}\right\rfloor+\bar{B}\right)$. Говорят, что дивизор $K+B n$-дополняем, если $n$-дополнение существует. В специальном случае $(X, B)$, когда $B=S=\sum S_{i}$ - приведенная граница, $n$-дополнение $K+S$ - дивизор $\bar{B} \in$ $|-n K-n S|$ такой, что $K+S+B$ lс, где $B:=\bar{B} / n$.

Оказьвается, что в случае 3 -мерного специального флипирующего сдутия $X \rightarrow Y$, с неприводимой границей $S, K+S n$-дополняем для $n \in\{1,2,3,4,6\}$ (см. [34; 5.12] или $[21 ; 19.6,19.8])$. Если $S$ приводима, то существование 3 -мерного специального флипа устанавливается легко (см., например, [34; 6.10], [21; 21.2]). Также легко устанавливается случай 1 -дополняемого $K+S$ с неприводимьм $S$. Для $n \geqslant 2$ задача сводится к случаю $n=2$ (см. $[34 ; 7.6]$, а также [21; Sections 6,21$])$.

Таким образом, следующий результат [34; 8.6-8.8], ([21; 22.10], а также [40]) является основным: для 3 -мерного 2 -дополняемого $K+S$ с неприводимым $S$ специальный флип существует.

Новое доказательство существования 3-мерных и доказательство существования 4-мерных klt логфлипов получено в недавней работе [37]. Оно основано на несколько других идеях: используется только редукция к допредельным флипам (вместо (i); см. определение 4.3 ниже) и специальньй обрыв (ii) (см. определение 4.6 ниже). Для конструкции допредельных и, в частности, специальных флипов вместо $n$-дополнений развивается техника конечно порожденных функциональных алгебр и индукция по размерности.

В этой главе мы воспроизведем, следуя [37] (см. также [10], [7]), доказательство в 3-мерном случае, основанное на этих новых идеях. Начнем с уже упомянутого понятия.

ОПРеДЕЛЕНИЕ 4.3. Пусть $(X, B)$ - логпара с $\mathbb{Q}$-факториальньм $X$ и $\mathbb{Q}$-границей $B$. Сдутие $\varphi:(X, B) \rightarrow Y$ называется (элементарньм) допредельнылм, если вьполнены следуюшие условия:

(i) $\varphi$ мало;

(ii) $(X, B) \mathrm{lt}$;

(iii) существует приведенная часть гранищы $S=\lfloor B\rfloor=\sum S_{i} \neq 0$, где $S_{i}$-простые дивизоры Вейля;

(iv) $\rho(X / Y)=1$;

(v) $-(K+B) \varphi$-обилен;

(vi) все $-S_{i} \varphi$-обильны. 
Допредельныцм (әлементарным) флипом называется диаграмма (4.1) со свойствами $\left(\mathrm{i}^{+}\right)-\left(\mathrm{iii}^{+}\right)$, как в определении 4.1.

ЗАмЕчАниЕ 4.4 . В $[37 ; 1.1]$ принято более обшее определение допредельного сдутия: $X$ не предполагается $\mathbb{Q}$-факториальным, $B-\mathbb{R}$-граница, сдутие - не обязательно проективное и малое, относительное число Пикара - произвольное. Обшность вызвана функториальностью такого общего определения при ограничениях на подмногооб-

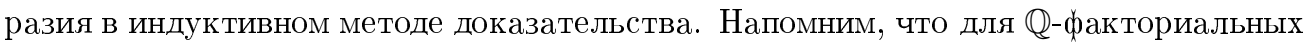
пар понятие логтерминальности и дивизориальной логтерминальности совпадают.

Следующий результат - редукция утверждения о существовании логфилипов к проблеме конечной порожденности некоторых относительных пучков алгебр - хорошо известен, начиная, по-видимому, с [33; 2.12] (см. также [11; Introduction]).

ПрЕДЛОЖЕНИЕ 4.5. Пусть $\varphi:(X, B) \rightarrow Y$ такое сдутие $c \mathbb{Q}$-границей $B$, как в определении 4.1 или 4.3. Тогда соответствующий флип существует, если и только если относительная $\mathscr{O}_{Y}$-алгебра

$$
\mathscr{R}_{X / Y}(K+B):=\bigoplus_{m \geqslant 0} \varphi_{*} \mathscr{O}_{X}(m(K+B))
$$

конечно порождена (отметим, что аналогичное утверждение справедливо и в самой общей ситуачии $[37 ; 3.32])$.

НАБросоК ДОКАЗАТЕЛЬСТвА. (Ср. с $[30 ; 1.6]$.$) Если флип \varphi^{+}:\left(X^{+}, B^{+}\right) \rightarrow Y$ существует, тогда $\mathscr{O}_{Y}$-алгебра

$$
\begin{aligned}
\mathscr{R}=\mathscr{R}_{X / Y}(K+B) & =\bigoplus_{m \geqslant 0} \varphi_{*} \mathscr{O}_{X}(m(K+B)) \\
& =\bigoplus_{m \geqslant 0} \mathscr{O}_{Y}\left(m\left(\varphi_{*}(K+B)\right)\right)=\bigoplus_{m \geqslant 0} \varphi_{*}^{+} \mathscr{O}_{X^{+}}\left(m\left(K_{X^{+}}+B^{+}\right)\right)
\end{aligned}
$$

конечно порождена, так как $\mathbb{Q}$-дивизор $K_{X^{+}}+B^{+} \varphi^{+}$-обилен. Промежуточные равенства следуют из того, что оба сдутия $\varphi$ и $\varphi^{+}$малы.

Обратно, предположим, что $\mathscr{R}=\mathscr{R}_{X / Y}(K+B)$ конечно порождена как $\mathscr{O}_{Y}$-алгебра. Алгебра $\mathscr{R}$ градуирована. Положим

$$
X^{+}:=\operatorname{Proj} \mathscr{R}_{X / Y}(K+B)
$$

с естественньм морфизмом $\varphi^{+}: X^{+} \rightarrow Y$. Ясно, что алгебра $\mathscr{R}$ целозамкнута (нормальна) как градуированная $[37 ; 4.8]$, а потому $X^{+}$нормально и $\varphi^{+}-$сдутие; покажем, что оно мало. Предположим, что это не так, т.е. существует дивизор $E^{+} \subset X^{+}$, исключительный для $\varphi^{+}$. В таком случае имеет место точная последовательность

$$
0 \rightarrow \mathscr{O}_{X^{+}} \rightarrow \mathscr{O}_{X^{+}}\left(E^{+}\right) \rightarrow \mathscr{K} \rightarrow 0
$$

с $\mathscr{K} \neq 0$. Приведем это к противоречию. Пусть $\mathscr{O}_{X+}(1)$ - относительно обильный пучок на $X^{+}$над $Y$, отвечаюший конструкции, т.е. градуированная алгебра $\mathscr{R}$ квазиизоморфна

$$
\mathscr{R}_{X^{+} / Y} \mathscr{O}_{X^{+}}(1)=\bigoplus_{m \geqslant 0} \varphi_{*}^{+} \mathscr{O}_{X^{+}}(m) .
$$


Тогда для $m \gg 0$ по теореме Серра $R^{1} \varphi_{*}^{+} \mathscr{O}_{X^{+}}(m)=0$ и гомоморфизм $\varphi^{+*} \varphi_{*}^{+}(\mathscr{K}) \otimes$ $\mathscr{O}_{X^{+}}(m) \rightarrow \mathscr{K} \otimes \mathscr{O}_{X^{+}}(m)$ сюръективен (порождаемость глобальными сечениями). Но в точной последовательности

$$
0 \rightarrow \varphi_{*}^{+} \mathscr{O}_{X^{+}}(m) \stackrel{\alpha}{\rightarrow} \varphi_{*}^{+}\left(\mathscr{O}_{X^{+}}\left(E^{+}\right) \otimes \mathscr{O}_{X^{+}}(m)\right) \rightarrow \varphi_{*}^{+}\left(\mathscr{K} \otimes \mathscr{O}_{X^{+}}(m)\right) \rightarrow 0
$$

гомоморфизм $\alpha$ является в действительности изоморфизмом по конструкшии, эффективности, исключительности $E^{+}$и равенству $\mathscr{R}=\mathscr{R}_{Y / Y} \varphi_{*}(K+B)$. Отсюда

$$
\varphi_{*}^{+}\left(\mathscr{K} \otimes \mathscr{O}_{X^{+}}(m)\right)=0 \Rightarrow \mathscr{K} \otimes \mathscr{O}_{X^{+}}(m)=0 \Rightarrow \mathscr{K}=0 .
$$

Противоречие.

Теперь, поскольку ч и $\varphi^{+}$малы, то $\operatorname{Div}_{\mathbb{Q}} X \simeq \operatorname{Div}_{\mathbb{Q}} X^{+}$. Пучок $\mathscr{O}_{X+}(1)$ обратим по конструкшии. Значит, $X^{+} \mathbb{Q}$-факториально и $\rho\left(X^{+} / Y\right)=1$. Утверждение об особенностях следует из (i) леммы 3.4 главы I.

Теперь воспроизведем редукцию проблемы существования klt логфлипа к проблеме существования допредельных флипов и специальному обрьву, следуя [34; § 6] [21; Section 18] (см. также [10]).

ОПРЕДЕЛЕНИЕ 4.6. Пусть

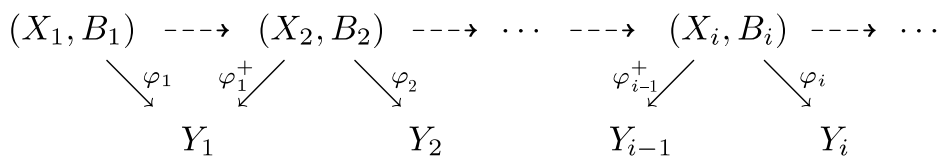

- любая последовательность логфлипов. Скажем, что для этой последовательности имеет место специальный обрыв, если существует такое $i_{0}$, что перестраиваемые кривые не пересекают приведенную часть гранищы $\left\lfloor B_{i}\right\rfloor$ всех lt пар $\left(X_{i}, B_{i}\right)$ с $i \geqslant i_{0}$.

ТЕОРема 4.7 (редукция к допредельным флипам [34; 6.4-5], [21; Section 18] или $[10 ; 1.7])$. Пусть $\varphi:(X, B) \rightarrow Y-$ малое klt логсдутие, как в определении 4.1, $\operatorname{dim} X=n \geqslant 3$. Тогда его klt логфлип существует, если:

(i) существует флип любого допредельного сдутия в размерности $n$;

(ii) специальный обрыв выполнен для любой последовательности логфлипов в той жее размерности п.

ДокАЗАТЕЛЬСтво-КОНстрУКция. Следуя [34; 6.4-5] и $[21 ; 18.12]$ (см. также $[10 ; 1.7])$, воспроизведем так назьваемую логперестраивающую процедуру, в результате которой малое klt сдутие $\varphi:(X, B) \rightarrow Y$ с помошью логПММ и условий (i) и (ii) теоремы будет модифицировано в относительную строго логминимальную модель $\bar{\psi}:\left(\bar{V}, \bar{B}=B_{\bar{V}}^{\log }\right) \rightarrow Y$ ("строго" означает, как обьчно, что $\bar{V} \mathbb{Q}$-факториально и проективно над $Y)$. Проверим, что $(\bar{V}, \bar{B})=\left(X^{+}, B^{+}\right)$и есть требуемьй логфлип. Действительно, по определению логобраза $B \frac{\log }{V}$ и лемме 2.4 модификация в $X^{+}$мала и $B^{+}=B_{X^{+}}^{\log }=B_{X^{+}}$. Значит, по (i) определения 4.1 сдутие $\bar{\psi}$ мало. После чего нетрудно вьвести $\left(\mathrm{ii}^{+}\right)$из $\mathbb{Q}$-факториальности $X$ и (ii), (iii) определения 4.1 (см. также комментарий 4.2). 
Процедура имеет два этапа: сначала несколько искуственно от klt сдутия мы переходим к lt относительной модели; затем в ней избавляемся от неисключительной приведенной части гранищы.

Начнем с перехода к lt относительной модели. Выберем эффективньй дивизор Картье $H$ на $Y$ и проективное раздутие $\sigma: V \rightarrow X$ со следующими свойствами:

a) $\psi=\varphi \circ \sigma: V \rightarrow Y$ - изоморфизм вне $\operatorname{Supp} H$;

b) логпара $\left(V, B_{V}^{\log }+H_{V}\right)$ имеет строго lt особенности ("строго" по прежнему означает, что $V \mathbb{Q}$-факториально и проективно над $Y$ ), где $H_{V}=\psi_{*}^{-1} H-$ собственный прообраз $H$;

c) если $\tau: Y^{\prime} \rightarrow Y$ - любое сдутие $\mathbb{Q}$-факториального и нормального многообразия $Y^{\prime}$, то простые компоненты $\tau^{*} H$ порождают относительное численное $\mathbb{R}$-пространство дивизоров $N^{1}\left(Y^{\prime} / Y\right)$.

В пояснениях нуждаются только утверждения а) и с). Для их вьполнения достаточно выбрать $H$ так, чтобы его носитель содержал все особенности многообразия $Y$, образ $\varphi(\operatorname{Exc} \varphi) \subset \operatorname{Supp} H$, а также в $\operatorname{Supp} H$ содержались бы некоторые простые дивизоры Вейля многообразия $Y$. Так что, как правило, дивизор $H$ приводим.

Применим логПММ с $B=B_{V}^{\log }+H_{V}$ к $V$ над $Y$. Встречаюшиеся при этом логфлипы с точностью до возмущения нерациональных кратностей границы будут допредельньми. Действительно, граница $B_{V}^{\log }+H_{V}$ содержит приведенный дивизор, который отрицательно пересекается с перестраиваемой флипом кривой $C$, так как $\psi^{*} H C=H \psi_{*} C=0$ и по с) существуют компоненты, пересекаюшие $C$ не по 0. Логфилип останется тем же, если мы выбросим все приведенные компоненты границы, пересекающие $C$ неотрицательно. Остальные приведенные компоненты дадут приведенную часть $S$, требуемую в определении $4.3 ;$ все остальное будет $B-S$. После возмушения $B-S$ можно предполагать $B \mathbb{Q}$-гранищей. По предположениям (i) и (ii) и известным результатам логПММ через конечное число шагов мы построим строго логминимальную модель $\bar{\psi}:\left(\bar{V}, B \frac{\log }{V}+H_{\bar{V}}\right) \rightarrow Y$ над $Y$.

Следующая задача - исключить $H_{\bar{V}}$ из гранищы $B_{\bar{V}}^{\log }+H_{\bar{V}}$. По построению $K_{\bar{V}}+$ $B \frac{\log }{V}+H_{\bar{V}}$ является $\bar{\psi}$-численно эффективным дивизором. Если $K_{\bar{V}}+B \frac{\log }{\bar{V}}$ является $\bar{\psi}$-численно эффективным, то $H_{\bar{V}}$ можно отбросить и $\bar{\psi}:(\bar{V}, B \bar{V} \log ) \rightarrow Y$ - требуемая строго логминимальная модель (как в начале доказательства). В противном случае сушествует кривая $C \subset \bar{V}$ над $Y$ с $H_{\bar{V}} C>0$ и $\left(K_{\bar{V}}+B_{\bar{V}}^{\log }\right) C<0$. Пусть $0 \leqslant \varepsilon \leqslant 1-$ наибольшее такое $\varepsilon$, что $K_{\bar{V}}+B \frac{\log }{V}+(1-\varepsilon) H_{\bar{V}}$ является $\bar{\psi}$-численно эффективным. Случай $\varepsilon=1$ уже рассмотрен. Пусть $0 \leqslant \varepsilon<1$. Тогда мы сможем его увеличить следующей процедурой. Для $0<\eta \ll \varepsilon$ если дивизор $K_{\bar{V}}+B \bar{V} \log +(1-\varepsilon-\eta) H_{\bar{V}}$ не $\bar{\psi}$-численно эффективен, то применим относительную логММП для $\left(\bar{V}, B \frac{\log }{V}+(1-\varepsilon-\eta) H_{\bar{V}}\right)$. Тогда логфлипы (логфлопы для $B_{\bar{V}_{i}}^{\log }+(1-\varepsilon) H_{\bar{V}_{i}}$ ) отвечают лучу, порождаемому кривой $C_{i}$ над $Y$ на $i$-м шаге. При этом

$$
0>\left(K_{\bar{V}_{i}}+B_{\bar{V}_{i}}^{\log }+(1-\varepsilon-\eta) H_{\bar{V}_{i}}\right) C_{i}=-\eta H_{\bar{V}_{i}} C_{i} \Rightarrow H_{\bar{V}_{i}} C_{i}>0 .
$$

Так как $0=\bar{\psi}_{i}^{*} H C_{i}=H_{\bar{V}_{i}} C_{i}+\sum \alpha_{k} E_{k} C_{i}, \alpha_{k} \geqslant 0$, то сушествует простой исключительный над $Y$ дивизор $E_{k}$ такой, что $E_{k} C_{i}<0$ и $C_{i} \subset E_{k} \subset \operatorname{Supp}\left\lfloor B_{\bar{V}_{i}}^{\log }\right\rfloor$, т.е. такие 
флипы тоже допредельны.

По предположению они сушествуют и обрьваются. Но здесь имеется тонкость с обрывом: увеличение $\varepsilon$ может происходить очень мальми порциями и не достигать 1 за конечное число шагов. Однако заметим, что построенная последовательность $\left(K_{\bar{V}}+B_{\bar{V}}^{\log }+(1-\varepsilon-\eta) H_{\bar{V}}\right)$ - флипов есть также последовательность $\left(K_{\bar{V}}+B_{\bar{V}}^{\log }\right)$-ф липов $($ c $\varepsilon+\eta=1)$, поскольку для любой перестраиваемой кривой $C_{i}$ в алгоритме редукции имеем $\left(K_{\bar{V}_{i}}+B_{\bar{V}_{i}}^{\log }+(1-\varepsilon-\eta) H_{\bar{V}_{i}}\right) C_{i}<0$ и $H_{\bar{V}_{i}} C_{i}>0$. Таким образом, через конечное число шагов мы придем к требуемой логминимальной модели.

Теперь рассмотрим вопрос о специальном обрьве.

УСЛОВНАЯ ТЕОРЕМА 4.8 (специальный обрыв, в предположении, что логПММ для lt логпар $(X, B)$ вьполняется в размерности $\leqslant n-1)$. Рассмотрим последовательность (4.2), где $\varphi_{i}: X_{i} \rightarrow Y_{i}-$ сдутие әкстремального луча $R_{i} c\left(K_{X_{i}}+B_{i}\right) R_{i}<0$ и $\varphi_{i}^{+}: X_{i}^{+}=X_{i+1} \rightarrow Y_{i}-$ его логфлип. Тогда в размерности $n$ после конечного числа логфлипов перестраиваемое подмножество (а также перестроенное подмножество) не пересекает $\left\lfloor B_{i}\right\rfloor$.

Прежде чем перейти к доказательству, необходимо ввести некоторые обозначения, дать несколько определений и прокомментировать их.

ОПРЕДЕЛЕНИЯ-ЗАМЕЧАНИЯ 4.9. (i) Пусть, как обычно, $\operatorname{LCS}(X, B)$ обозначает объединение логканонических центров, т.е. $\mathrm{c}_{X} E$ для простых b-дивизоров $E$ с $a(E, X, B)=-1$. Так как пара $(X, B) \mathrm{lt}$, то $\operatorname{LCS}(X, B)=\lfloor B\rfloor$ (напомним, что по определению логфлипа в гипотезе $1.11 X$ предполагается $\mathbb{Q}$-факториальным).

(ii) Кривые $C$ на $X_{i}$ (соответственно на $X_{i+1}=X_{i}^{+}$) будем называть иваемыми (соответственно перестроенными), если $\varphi_{i}(C)=$ pt. (соответственно $\varphi_{i}^{+}(C)=$ pt. $)$.

(iii) Пусть $S$ - логканонический центр логпары $(X, B)$. Тогда известно (см., например, [34; §3], [21; Section 16], а также [8]), что имеется lt логпара $\left(S, B_{S}\right)$, как и $(X, B)$, где $K_{S}+B_{S}=(K+B)_{\mid S}$ по формуле присоединения (в $\mathbb{Q}$-факториальной ситуации; см. замечание 4.4$)$.

(iv) Сдутие $f:(X, B) \rightarrow\left(X^{\prime}, B^{\prime}\right)$ логпар назьвается крепантнылм, если $K+B=$ $f^{*}\left(K_{X^{\prime}}+B^{\prime}\right)$, где $f_{*} B=B^{\prime}$.

$(\mathrm{v})$ Пусть $\varphi: U \rightarrow W-$ сдутие. Будем говорить, что оно типа $(\mathrm{D})$, если $\operatorname{Exc}(\varphi)$ содержит дивизор, и типа $(\mathrm{S})$, если $\varphi$ - малое сдутие. Пусть имеются два сдутия

$$
U \stackrel{\varphi}{\rightarrow} W \stackrel{\psi}{\leftarrow} V
$$

Тогда для них понятны обозначения (DD), (DS), (SS), (SD).

(vi) Пусть $B=\sum b_{i} D_{i}, 0 \leqslant b_{i} \leqslant 1$. Определим множество неотрицательных чисел $\mathbb{B}=\left\{b_{i}\right\}$ и положим

$$
S(\mathbb{B}):=\left\{1-\frac{1}{m}+\sum \frac{r_{i} b_{i}}{m} \mid m \in \mathbb{N}, r_{i} \in \mathbb{Z}_{\geqslant 0}\right\} .
$$


(vii) Пусть $S \subset \operatorname{LCS}(X, B)$ - логканонический центр. Определим сложность следующей формулой:

$$
d_{\mathbb{B}}\left(S, B_{S}\right):=\sum_{\alpha \in S(\mathbb{B})} \#\left\{E \mid a\left(E, S, B_{S}\right)<-\alpha, \mathrm{c}_{S} E \nsubseteq\left\lfloor B_{S}\right\rfloor\right\},
$$

где с $c_{S} E$ - центр простого b-дивизора $E$ многообразия $S$.

Ясно, что $d_{\mathbb{B}}\left(S, B_{S}\right)$ - конечное число, так как $\left(S \backslash \operatorname{Supp}\left\lfloor B_{S}\right\rfloor, B_{S}\right)$ klt и существует только конечное число простых $\mathrm{b}$-дивизоров с отрищательной дискрепантностью в klt случае (достаточно рассмотреть логразрешение).

ДОКАЗАТЕЛЬСТВО ТЕОРЕМЫ 4.8. (То же самое можно изЛожить в терминах контролируемых дискрепантностей; см. [39; Corollary 4].)

Шаг 1. После конечного числа логфлипов перестраиваемое множество не содержит логканонических центров. Действительно, число логканонических центров конечно. Дискрепантности над центрами, лежашими в перестраиваемом множестве, увеличиваются по лемме 2.4 , так что число центров уменьшается.

Следовательно, можно предполагать, что перестраиваемое подмножество не содержит логканонических центров для всех $\left(X_{i}, B_{i}\right)$. В этом случае модификации $\chi_{i}: X_{i} \rightarrow X_{i+1}$ индуцируют модификации $\left.\chi_{i}\right|_{S_{i}}: S_{i} \rightarrow S_{i+1}$ логканонических центров. При этом $a\left(E, S_{i}, B_{S_{i}}\right) \leqslant a\left(E, S_{i+1}, B_{S_{i+1}}\right)$ (например, по [38; Monotonicity]).

Шаг 2. Предположим, что $\chi_{i}: X_{i} \rightarrow X_{i+1}$ индуцирует логизоморфизм, т.е. изоморфизм логпар, на каждом логканоническом центре размерности $(d-1)$ в LCS для всех $i$. Тогда через конечное число логфлипов $\chi_{i}$ будет индуцировать логизоморфизм и на всех $d$-мерных центрах. Действительно, ясно, что $\chi_{i}$ индуцирует логизоморфизм на всех 0-мерных логканонических центрах. Ясно также, что если $\left.\chi_{i}\right|_{S_{i}}:\left(S_{i}, B_{S_{i}}\right) \rightarrow\left(S_{i+1}, B_{S_{i+1}}\right)-$ логизоморфизм, то $S_{i}$ не пересекает перестраиваемых кривых (см. шаг 3 ниже).

Далее доказательство делится на два случая:

a) когда пара $\left(\varphi_{i} S_{i},\left.\varphi_{i}^{+}\right|_{i+1}\right)$ имеет тип (SD) или (DD);

b) когда $\left(\varphi_{i}\left|S_{i}, \varphi_{i}^{+}\right| S_{i+1}\right)$ имеет тип (SS) или (DS).

В случае а) по лемме ниже уменьшается сложность, в случае b) обрьв происходит по логПММ в меньших размерностях.

Лемма 4.10. В любом случае имеет место неравенство для сложностей

$$
d_{\mathbb{B}}\left(S_{i}, B_{S_{i}}\right) \geqslant d_{\mathbb{B}}\left(S_{i+1}, B_{S_{i+1}}\right) .
$$

Более того, если $S_{i} \rightarrow T_{i} \leftarrow S_{i}^{+}=S_{i+1}$, где $T_{i}-$ нормализация $\varphi_{i} S_{i}$, имеет тип (SD) или (DD), то неравенство строгое. В этих случаях существует простой дивизор $E$ на $S_{i+1}$ такой, что

$$
a\left(E, S_{i}, B_{S_{i}}\right)<a\left(E, S_{i+1}, B_{S_{i+1}}\right)=-\alpha
$$

для некоторого $\alpha \in S(\mathbb{B})$. Стало быть, через конечное число логфлипов можно предполагать, ито $\left(\varphi_{i{ }_{S_{i}}}, \varphi_{i}^{+}{ }_{S_{i+1}}\right)$ имеют тип (SS) или (DS) для всех $i$. 
ДокАЗАТЕЛЬСТвО. Неравенство для сложностей вытекает из общего утверждения [38; Monotonicity] о неуменьшении дискрепантностей логквазифлипов (ср. с леммой 3.4(i)). При этом в случае (SD) и (DD) на $S_{i+1}$ имеется дивизор $E$ в $\operatorname{Exc}\left(\varphi_{i}^{+} S_{i+1}\right)$. Отождествляя $E$ c b-дивизором многообразия $S$, получаем неравенство $a\left(E, S_{i}, B_{S_{i}}\right)<a\left(E, S_{i+1}, B_{S_{i+1}}\right)$. При этом $a\left(E, S_{i+1}, B_{S_{i+1}}\right) \in S(\mathbb{B})$ по $[34 ; 3.10,4.2]$. Откуда вытекает строгое неравенство $d_{\mathbb{B}}\left(S_{i}, B_{S_{i}}\right)>d_{\mathbb{B}}\left(S_{i+1}, B_{S_{i+1}}\right)$.

ПРОДОЛЖЕНИЕ ДОКАЗАТЕЛЬСТВА ТЕОРЕМЫ 4.8. ПреДПоЛоЖИм, что каЖдая Последуюшая индуцированная модификация $\left(S_{i}, B_{S_{i}}\right)-\rightarrow\left(S_{i+1}, B_{S_{i+1}}\right)$ имеет тип (SS) или (DS). При этом $-\left(K_{S_{i}}+B_{S_{i}}\right)$ численно $\left.\varphi_{i}\right|_{S_{i}}$-обилен и $K_{S_{i+1}}+B_{S_{i+1}}$ численно $\varphi_{i}^{+} \mid S_{i+1}$-обилен, т.е. это - логфллип в смысле [36; Section 5], [39; Example 2], a согласно логПММ в версии второго автора [36; Section 5] такие флипы должны обрываться. Мы, однако, придерживаемся экстремальной и строгой lt версии, когда логфлипы экстремальны и строго lt и обрыв относится к ним (см. гипотезы 1.11 и 1.12 вьше). Поэтому нам надо еше немного поработать. Пусть $\left(S_{1}^{0}, B_{S_{1}^{0}}^{\log }\right) \rightarrow S_{1}-$ строгое lt раздутие, например, относительная строго логминимальная модель пары $\left(S_{1}, B_{S_{1}}\right)$ над $S_{1}$. Применим логПММ к морфизму $\left(S_{1}^{0}, B_{S_{1}^{0}}^{\log }\right) \rightarrow T_{1}$. По предположению через конечное число дивизориальных сдутий и логфллипов над $T_{1}$ мы придем к относительной логминимальной модели $\left(S_{2}^{0}, B_{S_{1}^{0}}^{\log }\right) \rightarrow T_{1}$.

Утверждается, что $\left(S_{2}^{0}, B_{S_{1}^{0}}^{\log }\right)=\left(S_{2}^{0}, B_{S_{2}^{0}}^{\log }\right) \rightarrow T_{1}$ - относительная строго логминимальная модель пары $\left(S_{2}, B_{S_{2}}\right)$ над $S_{2}$. Отметим, что проективность и $\mathbb{Q}$-факториальность для строгости получаем из логПММ, поскольку экстремальные дивизориальные сдутия и флипы сохраняют эти условия. По предложению 2.5 (iii) имеется сдутие $S_{2}^{0} \rightarrow S_{2}$, так как $\left(S_{2}, B_{S_{2}}^{\log }\right) \rightarrow T_{1}$ - относительная логканоническая модель логпар $\left(S_{1}, B_{S_{1}}\right)$ и $\left(S_{2}, B_{S_{2}}\right)$ в силу малости сдутия $S_{2} \rightarrow T_{1}$ и леммы 3.4 (i). Применим логПММ к морфизму $\left(S_{2}^{0}, B_{S_{2}^{0}}^{\log }\right) \rightarrow T_{2}$ и так далее. Через конечное число шагов процесс оборвется по логПММ, применимой по предположению.

Отметим, что пока мы не получили никакой информации о перестраиваемых кривых, которые не содержатся в $S_{i}$, но пересекают это подмногообразие. Это следующий шаг.

Шаг 3. По предыдушему можно считать, что после конечного числа логфилипов $S_{i}$ не содержит ни перестраиваемых, ни перестроенных кривых, т.е. $S_{i} \cong S_{i+1}$ для всех $i \gg 0$, и границы при этом сохраняются. Поэтому имеет место логизоморфизм $\left(S_{i}, B_{S_{i}}\right) \cong\left(S_{i+1}, B_{S_{i+1}}\right)$ для всех $i \gg 0$. Отсюда по лемме $3.4(\mathrm{i})$ о монотонности и формуле присоединения (ср. с доказательством $[34 ; 4.1])$ перестраиваемые кривые не пересекают такие $S_{i}$.

СлЕДСТвИЕ 4.11. Так как в размерности 3 логПММ выполнена, то специальный обрыв имеет место в размерности $n \leqslant 4$.

\section{§5. Редукция 3-мерного допредельного флипа в размерность 2}

5.1. В $\S 4$ было показано, что проблема сушествования klt логфлипа в размерности 3 сводится к сушествованию в той же размерности допредельного флипа, как в 
определении 4.3. При рациональности гранищы $B$ все это в любой размерности равносильно конечной порожденности дивизориальной алгебры

$$
\mathscr{R}_{X / Z}(K+B)=\bigoplus_{m \geqslant 0} \varphi_{*} \mathscr{O}_{X}(m(K+B)) .
$$

В дальнейшем, чтобы явно видеть, когда мы работаем в допредельном случае, вместо $B$ будем писать $S+B$, где $S$ - приведенная часть границы, а $B=\sum b_{i} D_{i}, 0 \leqslant b_{i}<1$, и все $b_{i}$ рациональны. Нетрудно все свести к случаю, когда $K+S+B$ plt, граница $B$ рациональна и $S$ - неприводимая поверхность. Поскольку наша цель - редукция 3 -мерного допредельного флипа в размерность 2 , то при ограничении на поверхность $S$ будем использовать присоединение: $(K+S+B)_{\mid S}=K_{S}+B_{S}$, где $B_{S}=\operatorname{Diff}(B)-$ гранища на $S$, так назьваемая дифферента (см. [34; $\S 3]$, [21; Section 16]). Нам не понадобится ее явньй вид, отметим только, что когда $K+S+B$ plt, как в нашем случае, то $K_{S}+B_{S}$ klt. Верно и обратное (см., например, [34;3.3-4], [21; 17.6]). Проблема сушествования флипа локальна по базе, так что многообразие $Z$ можно считать аффинным. В дальнейшем будем это предполагать. Напомним, что мы предполагаем $X$ $\mathbb{Q}$-факториальньм.

Отметим, что если $\varphi: X \rightarrow Z$ - допредельное сдутие, то $S$ содержит все $\varphi$-исключительное множество, потому что для любой кривой $C \subset \operatorname{Exc}(\varphi)$ имеем по определению $S C<0$.

Первьй шаг в доказательстве конечной порожденности алгебры $\mathscr{R}_{X / Z}(K+S+B)$ это определить ее ограничение $R_{S / Z}^{0}=\bigoplus R_{m}^{0}$ на $S$, где

$$
R_{m}^{0}:=\operatorname{Im}\left(H^{0}(X, m(K+S+B)) \rightarrow H^{0}\left(S, m\left(K_{S}+B_{S}\right)\right)\right) .
$$

Легко показать, что алгебра $\mathscr{R}_{X / Z}(K+S+B)$ конечно порождена, если и только если ограничение $R_{S / Z}^{0}$ конечно порождено (см. предложение 5.4 ниже). Однако $R_{S / Z}^{0}$ в обшем случае уже не является дивизориальной алгеброй, как $\mathscr{R}_{X / Z}(K+S+B)$, поскольку предыдушие ограничения не являются, вообше говоря, сюръективными. В связи с этим в $[37 ; 4.10]$ вводится понятие псевдо-дивизориальной алгебры и, более того, так как линейные системы $|m(K+S+B)|$ и их ограничения на $S$ имеют, вообще говоря, базисные подмножества, то приходится следить за их разрешениями, в связи с чем вводится язьк b-дивизоров (бирациональных дивизоров). На этом языке алгебра $R_{S / Z}^{0}$ (точнее, ее целое замыкание) является псевдо-b-дивизориальной алгеброй (pbd-алгеброй). Ниже мы дадим точные определения.

В общем случае нет оснований утверждать, что pbd-алгебра $R_{S / Z}^{0}$ конечно порождена. В [37; Section 4] вводятся два (необходимых) сушественных условия, при которых это удается доказать для индуцированного сдутия $S / Z$ (пока только в размерности $\leqslant 2$, в обшем случае - это гипотеза):

а) ограниченность (см. определение 5.19 ниже);

b) логканоническое асимптотическое (или lса-) насьшение (см. определение 5.23 ниже).

pbd-алгебры с условиями a) и b) для слабых логсдутий Фано $\varphi: X \rightarrow Z$ (т.е. пара $(X, B) \mathrm{klt}$, а дивизор $-(K+B)$-численно эффективен; $\varphi$-объемность вытекает из 
бирациональности $\varphi$ ) назьваются FGA-алгебрами: основная гипотеза - все FGA-алгебры конечно порождены (см. гипотезу 5.25 ниже).

Доказательство этой гипотезы даже в 1-мерном случае требует некоторых утверждений из теории рациональных приближений (см. [37; 4.41], [3; 2.1], [7; 4.10]). В 2-мерном случае сушественно используется бирациональная геометрия поверхностей. Основньм является утверждение (см. теорему 6.4 ниже), что насьшенный подвижный b-дивизор $\mathbb{M}$ на неособой поверхности не имеет базисных точек, т.е. свободен. Очень важным также является условие lса-насышения, и, конечно, сушественно используется язык функциональных алгебр и b-дивизоров. Далее мы будем придерживаться замечательного обзора А. Корти [7].

ОПРЕДЕЛЕНИЕ 5.2. Пусть $\varphi: X \rightarrow Z$ - сдутие на аффинное многообразие $Z$ с координатным кольцом $A=H^{0}\left(Z, \mathscr{O}_{Z}\right)$. Функииональная алгебра на $X / Z$ - это градуированная $A$-подалгебра $V \subset k(X)[T]$, т.е. $V=\bigoplus_{i \geqslant 0} V_{i}, V_{0}=A, V_{i} \subset k(X)$ и $V_{i} V_{j} \subset V_{i+j}$, где умножение определяется как обычное произведение функций. Кроме того, предполагается, что каждый $A$-модуль $V_{i}$ конечно порожден над $A$ (когерентен). Функциональная алгебра ограничена, если существует целый дивизор Вейля $D$ на $X$ такой, что каждьй $V_{i} \subset H^{0}(X, i D)$. В таком случае будем говорить, что $V$ ограничена дивизором $D$.

Прорежсенная подалгебра - это градуированная $A$-подалгебра вида $V^{(d)}:=$ $\bigoplus_{i} V_{i d}$ для фиксированного натурального числа $d \neq 0$.

Хорошо известно, что градуированная функциональная алгебра конечно порождена, если и только если конечно порождена ее прореженная алгебра [37; 4.6]. (Однако это неверно для более обших градуированных алгебр [37; 4.5].) В дальнейшем, как правило, мы будем рассматривать функциональные алгебры с точностью до прореживания и даже квазиизоморфизма (часто даже не упоминая об этом явно).

ОПРЕДЕЛЕНИЕ 5.3. Пусть $X$ - нормальное многообразие, а $S \subset X$ - неприводимое нормальное подмногообразие коразмерности 1 . Пусть $\mathscr{O}_{X, S} \subset k(X)$ - локальное кольцо регулярных функций в обшей точке подмногообразия $S$, а $\mathfrak{m}_{X, S} \subset \mathscr{O}_{X, S}$ - максимальньй идеал последнего. Тогда $k(S)=\mathscr{O}_{X, S} / \mathfrak{m}_{X, S}$ - поле рациональных функций на $S$.

Функциональная алгебра $V=\bigoplus V_{i}$ назьвается регулярной вдоль $S$, если вьполнены следуюшие условия:

(i) каждьй $V_{i} \subset \mathscr{O}_{X, S} \subset k(X)$;

(ii) $V_{1} \not \subset \mathfrak{m}_{X, S}$.

Если $V$ регулярна вдоль $S$, то вычетная алгебра $V^{0}=V_{\left.\right|_{S}}=\operatorname{res}_{S} V$ является функциональной алгеброй $V^{0}=\bigoplus V_{i}^{0}$, где

$$
V_{i}^{0}=\operatorname{Im}\left(V_{i} \rightarrow k(S)\right) \text {. }
$$

Если $V$ ограничена дивизором Картье $D$ и $\operatorname{Supp} D \not \subset S$, то, очевидно, вычетная алгебра $V^{0}=\operatorname{res}_{S} V$ тоже ограничена, например, дивизором $D_{\mid S}$.

Пусть $(X, S+B)$ - plt пара и $\varphi: X \rightarrow Z$ - флипирующее сдутие. Как мы уже знаем, флип сушествует, если и только если $A$-алгебра $\mathscr{R}_{X / Z}(K+S+B)$ конечно порождена. 
Эта алгебра является функциональной с естественными вложениями $H^{0}(X, i(K+S+$ $B)) \subset k(X)$, связанными с кратными дивизорами $i(K+S+B)$. Так как $\rho(X / Z)=1$, то вместо $K+S+B$ можно взять любой $\varphi$-отрицательный $\mathbb{Q}$-дивизор $D$. Тогда $D \sim \mathbb{Q} r(K+S+B)$ для некоторого положительного числа $r \in \mathbb{Q}$, так что дивизориальные алгебры $\mathscr{R}_{X / Z}(K+S+B)$ и $\mathscr{R}_{X / Z} D$ квазиизоморфны, т.е. имеют общую прореженную подалгебру с точностью до умножения на рациональную функцию $s \in k(X)$, точнее, на $s^{i}$ для элементов алгебры степени $i$. В случае допредельного сдутия $S \sim_{\mathbb{Q}} D$ для некоторого из этих дивизоров $D$ и, более того, $S \not \subset \operatorname{Supp} D$ для подходяшего $D$.

ПРЕДЛОЖЕНИЕ 5.4 [37; 3.43], [7; 3.1.5]. Пусть $\varphi: X \rightarrow Z-$ малое сдутие $u$ $S$ - простой дивизор на $X$, а $D-$ такой $\mathbb{Q}$-дивизор Вейля, что $D \sim_{\mathbb{Q}} S$ и $S \not \subset$ Supp D. Тогда D-флип существует, если и только если вычетная алгебра $R^{0}=$ $\operatorname{res}_{S} \mathscr{R}_{X / Z} D$ конечно порождена.

ДоКАЗАТЕЛЬСТВо. Как и вьше, достаточно установить конечноую порожденность $\mathscr{R}(D)=\mathscr{R}_{X / Z} D$. С точностью до квазиизоморфизма мы можем предполагать, что $D \sim S$, т.е. сушествует рациональная функция $t \in k(X)$ такая, что $S=D+(t)$, где $(t)$ - дивизор нулей и полюсов функции $t ; t \in \mathscr{R}_{1}(D)$. По нашим предположениям алгебра $\mathscr{R}(D)$ регулярна вдоль $S$; если $S$ не нормален, то вычетную алгебру следует рассматривать на нормализации $S / Z$ (в приложениях $S$ нормален, что следует, например, из условия plt). Покажем, что ядро ограничения $\mathscr{R}(D) \rightarrow R^{0}$ является главньм идеалом, порожденньм $t$. Действительно, пусть $u \in \mathscr{R}_{i}(D) \subset k(X)$ ограничивается в $0 \in R^{0}$. Это означает, что $(u)+i D \geqslant 0$ и и обращается в нуль на $S$, т.е. $(u)+i D-S \geqslant 0$. Следовательно, мы можем представить $u=t u^{\prime}$, где $\left(u^{\prime}\right)+(i-1) D=(u)+D-S+(i-1) D \geqslant 0$. Иными словами, $u^{\prime} \in \mathscr{R}_{i-1}(D)$ и $u \in(t)=\mathscr{R}(D)(t)$. Значит, $R^{0}$ конечно порождена, если и только если $\mathscr{R}(D)$ конечно порождена.

Теперь обратимся к b-дивизорам. Напомним, что их возникновение связано с рассмотрением линейных систем с базисными точками вместе со всеми возможными разрешениями, т.е. цепочками собственных моделей над $X$.

ОПРЕДЕЛЕНИЕ 5.5 [9]. Пусть $X$ - нормальное многообразие. Цельй b-дивизор многообразия $X$ - это элемент обратного предела

$$
\mathbb{D} \in \lim _{Y \rightarrow X} \operatorname{Div} Y
$$

где проективньй предел берется по всем собственным бирациональным морфизмам $f: Y \rightarrow X$ с гомоморфизмом $f_{*}: \operatorname{Div} Y \rightarrow \operatorname{Div} X$.

b-дивизоры с кратностями в $\mathbb{Q}$ и $\mathbb{R}$ определяются аналогично. Группа b-дивизоров многообразия $X$ обозначается через $\operatorname{Div}(X)$. Гомоморфизм прямого образа $f_{*}: \operatorname{Div}(Y) \rightarrow \operatorname{Div}(X)$ является изоморфизмом, так что мы можем отождествлять b-дивизоры многообразия $X$ c b-дивизорами многообразия $Y$.

След b-дивизора $\mathbb{D}$ на модели $Y \rightarrow X$ (или любом собственном бирациональном преобразовании) - это обычный дивизор (Вейля) на $Y$

$$
\mathbb{D}_{Y}:=\sum_{\substack{F-\text { простые } \\ \text { дивизоры на } Y}} d_{F} F,
$$


если $\mathbb{D}=\sum d_{F} F$, где $F$ - простые b-дивизоры, т.е. дивизориальные нормирования

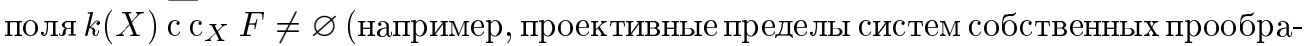
зов обычных простых дивизоров $F$ с $X$ на всех моделях $Y \rightarrow X)$. Простой b-дивизор для простого дивизора $F \in \operatorname{Div} X$ будем иногда обозначать через $\underline{F}$. Для $\mathbb{Q}$ - (или $\mathbb{R}$-) Картье дивизора $D$ на $X$ через $\bar{D}$ обозначается его Картье замькание со следом $\bar{D}_{Y}=f^{*} D$ на всех моделях $f: Y \rightarrow X$.

ПримеРы 5.6. 1. Пусть $f \neq 0 \in k(X)$ - рациональная функция, тогда определен eе b-дивизор

$$
\operatorname{div}(f):=\sum \operatorname{mult}_{E}(f) E,
$$

где $E$ - простой b-дивизор многообразия $X$ и $\operatorname{mult}_{E}(f)-$ кратность функции $f$ вдоль $E$ (напомним, что $E$ отождествляется с дискретньм нормированием поля $k(X)$ ).

2. На нормальном многообразии $X$ определен канонический $\mathrm{b}-\partial и в и з о р ~ \mathbb{K}=\operatorname{div}(\omega)$, где $\omega \neq 0 \in \Omega_{k(X)}$ - рациональная дифференциальная форма старшей степени на $X$. Действительно, на (неособой) модели $f: Y \rightarrow X$ имеем

$$
\mathbb{K}_{Y}=\left(f^{*} \omega\right)=K_{Y}
$$

так что $f_{*} \mathbb{K}_{Y}=f_{*}\left(f^{*} \omega\right)=(\omega)=K_{X}$, т.е. вьполнено условие согласованности в проективном пределе.

3. Пусть $X$ - нормальное многообразие и $D=\sum d_{i} D_{i}-\mathbb{R}$-дивизор на $X$ такой, что $K+D \mathbb{R}$-Картье. Тогда для пары $(X, D)$ определен b-дивизор дискрепантностей $\mathbb{A}=\mathbb{A}(X, D)=\mathbb{K}-\overline{(K+D)}$ со следами $\mathbb{A}_{Y}$ на моделях $f: Y \rightarrow X$, где $\mathbb{A}_{Y}$ также определяется формулой

$$
K_{Y}=f^{*}(K+B)+\mathbb{A}_{Y}
$$

Для b-дивизоров, как и для обычных дивизоров, определены пучки $\mathscr{O}_{X}$-модулей, которые, вообше говоря, не обязательно когерентны.

ОПРЕДЕЛЕНИЕ 5.7. Пусть $\mathbb{D}=\sum d_{F} F-$ b-дивизор многообразия $X$ ( $X$ может быть аффинньм). Тогда определен пучок $\mathscr{O}_{X}$-модулей $\mathscr{O}_{X}(\mathbb{D})$ на $X$ : для каждого открытого подмножества $U \subset X$

$$
\begin{aligned}
\Gamma\left(U, \mathscr{O}_{X}(\mathbb{D})\right)=\{f & \in k(X) \mid \operatorname{mult}_{F} f+d_{F} \geqslant 0 \\
& \text { для всех простых b-дивизоров } F \text { многообразия } X\} .
\end{aligned}
$$

Из определения видно, что $\mathscr{O}_{X}(\mathbb{D})$ - подпучок постоянного пучка $k(X)$ и даже пучка $\mathscr{O}_{X}\left(\mathbb{D}_{X}\right)$. Отметим, что $H^{0}(X, \mathbb{D}):=H^{0}\left(X, \mathscr{O}_{X}(\mathbb{D})\right) \subset H^{0}\left(X, \mathbb{D}_{X}\right)$, т.e. b-дивизор $\mathbb{D}$ выделяет линейную (под)систему в полной линейной системе дивизора $\mathbb{D}_{X}$. Иногда это выделение задается базисными условиями, что и явилось отправной точкой для введения b-дивизоров.

Пучок $\mathscr{O}_{X}(\mathbb{D})$ может быть когерентным, даже если $\mathbb{D}$ задается бесконечньм числом нетривиальных базисных условий. Это возможно, например, тогда, когда имеется конечный дивизор $D^{\prime}=\mathbb{D}_{Y}$ на некоторой модели $f: Y \rightarrow X$ такой, что $\mathscr{O}_{X}(\mathbb{D})=$ $f_{*} \mathscr{O}_{Y}\left(D^{\prime}\right)$ (стабилизация). 
Центральньм местом конструкщии 3-мерного допредельного флипа является поиск такой фиксированной модели $f: Y \rightarrow X$, что для некоторой бесконечной последовательности b-дивизоров $\mathbb{D}_{i}$ многообразия $X$ вьполнено условие $\mathscr{O}_{X}\left(\mathbb{D}_{i}\right)=f_{*} \mathscr{O}_{Y}\left(\mathbb{D}_{i Y}\right)$.

Следующая лемма дает некоторые естественные условия, гарантирующие когерентность $\mathscr{O}_{X}(\mathbb{D})$ в некоторых случаях. Этот результат будет использован в дальнейшем.

Лемма 5.8 [37; Proof of 4.46], [7; 3.2.7]. Пусть $X$-неособое многообразие, $D=$ $\sum d_{i} D_{i}-\mathbb{R}$-дивизор, носитель которого $\sum D_{i}-$ дивизор с (простьми) нормальнымми пересечениями. Пусть $\mathbb{A}=\mathbb{A}(X, D)$ - b-дивизор дискрепантностей $u$ $f: Y \rightarrow X$ нормальное раздутие. Запишем

$$
\left\lceil\mathbb{A}_{Y}\right\rceil=f^{*}\left\lceil\mathbb{A}_{X}\right\rceil+\sum e_{i} E_{i}
$$

где $E_{i}-$ исключительные дивизоры. Тогда все $e_{i} \geqslant 0$.

Первым следствием этого результата является

СлЕДСТВИЕ 5.9 [37; 4.47], [7; 3.2.8]. Если $X$ - нормальное многообразие, $D=$ $\sum d_{i} D_{i}-\mathbb{R}$-дивизор, для которого определен $b$-дивизор дискрепантностей $\mathbb{A}=$ $\mathbb{A}(X, D)$, то $\mathscr{O}_{X}(\lceil\mathbb{A}\rceil)$ - когерентный пучок.

Отметим, что в этом следствии $D-\mathbb{R}$-дивизор (при условии, что $K+D-\mathbb{R}$-Картье), т.е. не используется никакое из предположений об особенностях пары $(X, D)$.

Для доказательства следствия достаточно выбрать логразрешение и воспользоваться предыдущей леммой.

Следуюшее определение обобщает на b-дивизоры понятия, естественные для обычных дивизоров.

ОПРЕДЕЛЕНИЕ 5.10. b-дивизор $\mathbb{D} \in \mathbf{D i v}(X)$ назьвается b-Картье (соответственно b-свободньм, b-численно эффективным, b-полуобильным и т. п.), если сушествует модель $Y \rightarrow X$ такая, что $D=\mathbb{D}_{Y}$ является дивизором Картье (соответственно свободным, численно эффективным, полуобильным и т.п.) и $\mathbb{D}=\bar{D}$. Аналогично для $\mathbb{D} \in \operatorname{Div}(X) \otimes \mathbb{Q}, \operatorname{Div}(X) \otimes \mathbb{R}$.

Рассмотрим теперь важнейшее, введенное впервые в [37; 4.33], понятие насьшения дивизоров и b-дивизоров.

ОПРЕДЕЛЕНИЕ 5.11. (i) Подвижной частью (Эффективного) целого дивизора $D$ на $X$ является дивизор

$$
\operatorname{Mov} D=D-F \text {, }
$$

где $F=\operatorname{Fix}|D|-$ неподвижная часть полной линейной системы $|D|$.

(ii) Пусть $D$ как и вьше и $C-\mathbb{R}$-дивизор на $X$ такой, что $\lceil D+C\rceil$ эффективен с точностью до линейной эквивалентности, т.е. $|\lceil D+C\rceil| \neq \varnothing$. Говорят, что $D C$-насыщен, если Mov $\lceil D+C\rceil \leqslant D$. Дивизор $D C$-насыщен всегда, когда $|\lceil D+C\rceil|=\varnothing$.

(iii) (Эффективньй) b-дивизор $\mathbb{D}$ многообразия $X$ назьвается $\mathbb{C}$-насыциеннылм, ес-

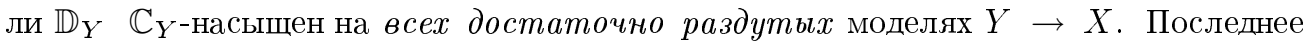
означает, что сушествует конкретное раздутие $Y \rightarrow X$, на котором вьполнено насышение, и оно также выполнено на всех последуюших раздутиях $Y^{\prime} \rightarrow Y \rightarrow X$. Если 
$\mathbb{C}=\mathbb{A}(X, B)$ - b-дивизор дискрепантностей логпары $(X, B)$, то будем говорить, что $\mathbb{D}$ логканонически (lc-) насыцен, точнее - логканонически (lc-) насышен над $(X, B)$.

(iv) (Эффективный) b-дивизор $\mathbb{D}$ многообразия $X$ назьвается исключительно насыщенныцм, если он $\mathbb{E}$-насыщен для всех исключительных на $X \mathbb{R}$-b-дивизоров $\mathbb{E}$ многообразия $X$.

ПримеР 5.12. Картье замыкание $\bar{D} \mathbb{Q}$-Картье целого дивизора Вейля $D$ исключительно насышенно. Это следует из хорошо известного утверждения о том, что для всех моделей $f: Y \rightarrow X$

$$
f_{*} \mathscr{O}_{Y}\left(\left[f^{*} D+\sum e_{i} E_{i}\right\rceil\right)=f_{*} \mathscr{O}_{Y}(\bar{D})=\mathscr{O}_{X}(D),
$$

где $E_{i}$ исключительны и все $e_{i} \geqslant 0$.

Для доказательства какого-нибудь утверждения о насьшении b-дивизора прежде всего надо найти конкретную модель $Y \rightarrow X$, на которой вьполнено насьщение. Следуюшая лемма подсказывает условия, при которых насьшение с модели $Y \rightarrow X$ может быть продолжено на все последуюшие раздутия $Y^{\prime} \rightarrow Y \rightarrow X$. Она легко выводится из леммы 5.8 .

Лемма $5.13[37 ; 6.36],[7 ; 3.3 .8]$. Пусть $(X, D)$ - пара $c \mathbb{R}$-дивизором $D$ на $X u$ $K+D \mathbb{R}$-Картье. Пусть $\mathbb{D}$ - b-дивизор многообразия $X$ и $Y \rightarrow X-$ модель, для которых выполнены следующие условия:

(i) $Y-$ неособо и $\mathbb{D}_{Y}+\mathbb{A}_{Y}-$ дивизор с (простыци) нормальныли пересечениями;

(ii) $\mathbb{D}=\overline{\mathbb{D}_{Y}}$.

Тогда $\mathbb{A}$-насыщение, где $\mathbb{A}=\mathbb{A}(X, D)$, выполнено на $Y$, если и только если оно вьполнено для любого последующего раздутия $Y^{\prime} \rightarrow Y \rightarrow X$, m.е.

$$
\operatorname{Mov}\left\lceil\mathbb{D}_{Y}+\mathbb{A}_{Y}\right\rceil \leqslant \mathbb{D}_{Y} \Longleftrightarrow \operatorname{Mov}\left\lceil\mathbb{D}_{Y^{\prime}}+\mathbb{A}_{Y^{\prime}}\right\rceil \leqslant \mathbb{D}_{Y^{\prime}}
$$

Следуюшее очевидное утверждение полезно в нашей ситуации при ограничении на $S$.

Лемма 5.14. Пусть $(X, S+B)$ - plt логпара $c$ b-дивизором дискрепантностей $\mathbb{A}=\mathbb{A}(X, S+B)$. Если b-дивизор $\mathbb{D}$ исключительно насыщен, то он $(\mathbb{A}+\underline{S})$ насыщен.

ДокАЗАТЕЛЬСТво. Положительная часть $\mathrm{b}$-дивизора $\mathbb{A}+\underline{S}$ исключительна на $X$.

Возврашаясь к нашей задаче о конечной порожденности дивизориальной алгебры $\mathscr{R}_{X / Z}(K+S+B)=\bigoplus_{m \geqslant 0} H^{0}(X, m(K+S+B))$, заметим, что дивизоры $m(K+S+B)$ можно заменить их подвижными частями $\operatorname{Mov}(m(K+S+B))$ : от этого $A$-подмодули функций $H^{0}(X, m(K+S+B))$ не изменятся, что не скажется на вопросе конечно порожденности. Однако если отбросить неподвижные части, то алгебра не будет, вообще говоря, дивизориальной. С учетом базисных условий необходимо приходим к pbd-алгебре. Для этого из b-дивизора нужно выделить его подвижную часть и с последовательностью подвижных частей связать функциональную алгебру, чем мы сейчас и займемся. 
ОПРЕДЕЛЕНИЕ 5.15. (i) Цельй b-дивизор $\mathbb{D}$ называется подвижнылм, если он b-свободен.

(ii) b-подвижсная часть $\mathbb{Q}$-Картье целого (эффективного) дивизора $D$ на $X$ относительно $A$-подмодуля $L \subset H^{0}(X, D)$ (с точностью до линейной эквивалентности $D \in L)$ есть b-дивизор $\operatorname{Mov}_{L} D$ со следом $\left(\mathbb{M o v}_{L} D\right)_{Y}=f^{*} D-\operatorname{Fix} f^{*}|L|$ на каждой модели $f: Y \rightarrow X$.

$($ При $\mathbb{Q}$-факториальности $X$ b-подвижная часть определена для любого целого $D$ на $X$.) Подвижная часть $\operatorname{Mov}_{L} D$ является подвижным b-дивизором (это равносильно сушествованию разрешения базисного множества не обязательно полных линейных систем).

b-подвижная часть характеризуется формулой (см. $[37 ; 4.15],[10 ; 2.5],[7 ; 3.4 .4])$

$$
\operatorname{mult}_{E} \operatorname{Mov}_{L} D=-\min _{\varphi \in L} \operatorname{mult}_{E} \varphi
$$

для всех простых b-дивизоров $E$ многообразия $X$, где $\operatorname{mult}_{E}(\cdot)$ обозначает кратность вдоль b-дивизора $E$. Правая часть как будто бы не зависит от $D$. Однако это не так, поскольку вложение $L \subset k(X)$ зависит от $D$ (по определению $H^{0}(X, D)=\{\varphi \in k(X) \mid$ $(\varphi)+D \geqslant 0\})$.

В случае полных линейных систем, когда $L=H^{0}(X, D)$, будем писать просто $\mathbb{M o v} D$ для b-подвижной части $D$. Она характеризуется формулой

$$
(\operatorname{Mov} D)_{Y}=\operatorname{Mov}\left\lfloor f^{*} D\right\rfloor
$$

на всех моделях $f: Y \rightarrow X$.

ЛЕмма 5.16. b-подвижная часть $\mathbb{M o v} D \mathbb{Q}$-Картье челого дивизора Вейля $D$ на $X$ исключительно насыщена.

ДокАЗАТЕльство. Это следует из характеризации $(\mathbb{M o v} D)_{Y}=\operatorname{Mov}\left\lfloor f^{*} D\right\rfloor$ на моделях $Y \rightarrow X$.

Конечно, произвольньй подвижньй b-дивизор $\mathbb{M}$ не обязательно исключительно насьшен, как показывают простые примеры, например, раздутие точки на плоскости.

Переходим к обсуждению важного результата: при подходяших условиях исключительное насыщение над $X$ влечет lc-насьшение над $S$. В общем случае не ясно, как определять ограничение произвольного b-дивизора $\mathbb{D}$ на $S$, даже в случае, когда $S$ не содержится в носителе дивизора $\mathbb{D}_{X}$. Однако это можно сделать в случае подвижных b-дивизоров.

ОПРЕДЕЛЕНИЕ 5.17 [37; 7.1], [7; 3.5.1]. Пусть $\mathbb{M}$ - подвиЖньй b-дИвИзор многообразия $X$ и $S \subset X-$ простой дивизор, не содержашийся в носителе $\mathbb{M}_{X}$. Определим подвижное ограничение $\mathbb{M}^{0}=\operatorname{res}_{S} \mathbb{M}$ на $S$ (точнее на нормализацию $S$, когда дивизор $S$ как многообразие не нормален) следуюшим образом: выберем модель $Y \rightarrow X$ такую, что след $\mathbb{M}_{Y}$ свободен и $\mathbb{M}=\overline{\mathbb{M}_{Y}}$, и пусть $S^{\prime}-$ собственньй прообраз $S$ (и его нормализация при ненормальности) на $Y$. Тогда положим

$$
\operatorname{res}_{S} \mathbb{M}=\overline{\mathbb{M}_{H_{S^{\prime}}}}
$$


Иначе говоря, $\operatorname{res}_{S} \mathbb{M}$ - это Картье замыкание обычного ограничение $\mathbb{M}_{H_{S^{\prime}}}$, дивизора Картье. Так как Div $S^{\prime}=\operatorname{Div} S$, то мы получили подвижньй b-дивизор $\mathbb{M}^{0}=\operatorname{res}_{S} \mathbb{M}$ многообразия $S$. Легко убедиться, что подвижное ограничение не зависит от выбора модели $Y \rightarrow X$.

Отметим, что подвижное ограничение аддитивно, т.е. удовлетворяет условию

$$
\left(\mathbb{M}_{1}+\mathbb{M}_{2}\right)^{0}=\mathbb{M}_{1}^{0}+\mathbb{M}_{2}^{0} .
$$

Лемма 5.18 [37; 4.50], [7; 3.5.4]. Пусть $\varphi:(X, S+B) \rightarrow Z$ - plt cлабое cдyтие Фано на $Z$, т.е. $K+S+B$ plt $u-(K+S+B)$ ч-численно әффективен ( $\varphi$-облемность вытекает из бирачиональности $X \rightarrow Z)$. Пусть $\mathbb{M}$ - подвижсный b-дивизор многообразия $X$, и предположим, что $S$ не содержится в носителе дивизора $\mathbb{M}_{X}$.

Пусть, как обьчно, $\mathbb{A}=\mathbb{A}(X, S+B)$ - b-дивизор дискрепантностей, и предположим, что $\mathbb{M}$ является $(\mathbb{A}+\underline{S})$-насыщенным (по лемме 5.14 это так, если $\mathbb{M}$ исключительно насыщен). Тогда подвижное ограничение $\mathbb{M}^{0}=\operatorname{res}_{S} \mathbb{M}$ является lс-насыщенным над $\left(S, B_{S}\right)$, т.е. $\mathbb{A}\left(S, B_{S}\right)$-насышенным.

ДокАЗАТЕльство. Пусть $f: Y \rightarrow X$-логразрешение пары $(X, S+B), F_{i}-f$-исключительные простые дивизоры, а $D_{j}^{\prime}$ и $S^{\prime}$ - собственные прообразы простых компонент границы $B$ и дивизора $S$ соответственно. Модель $Y \rightarrow X$ можно выбрать достаточно раздутой так, чтобы вьполнялись следующие условия:

a) $\mathbb{M}_{Y}$ свободен и $\mathbb{M}=\overline{\mathbb{M}_{Y}}$;

b) на $Y$ вьполнено условие $\mathbb{A}^{\prime}$-насьшения $\mathbb{M}$, т.е.

$$
\operatorname{Mov}\left\lceil\mathbb{M}_{Y}+\mathbb{A}_{Y}^{\prime}\right\rceil \leqslant \mathbb{M}_{Y}
$$

где $\mathbb{A}^{\prime}=\mathbb{A}+\underline{S}$.

Запишем

$$
K_{Y}=f^{*}(K+S+B)-S^{\prime}-\sum b_{j} D_{j}^{\prime}+\sum a_{i} F_{i} .
$$

Тогда по определению дискрепантностей

$$
\mathbb{A}_{Y}=-S^{\prime}-\sum b_{j} D_{j}^{\prime}+\sum a_{i} F_{i} .
$$

Покажем, что ограничение $\mathbb{M}^{0}=\operatorname{res}_{S} \mathbb{M}$ lc-насьшено. Для этого проверим прежде всего, что насьщение вьполнено на модели $S^{\prime} \rightarrow S$. Обозначим $\mathbb{A}^{0}=\mathbb{A}\left(S, B_{S}\right)$. По формуле присоединения $\mathbb{A}_{S^{\prime}}^{0}=\left.\mathbb{A}_{Y}^{\prime}\right|_{S^{\prime}}$. С другой стороны, по теореме обрашения в нуль Каваматы-Фивега имеем

$$
H^{1}\left(Y,\left\lceil\mathbb{M}_{Y}+\mathbb{A}_{Y}\right\rceil\right)=H^{1}\left(Y, K_{Y}+\left\lceil-f^{*}(K+S+B)+\mathbb{M}_{Y}\right\rceil\right)=0
$$

поскольку по условию $-(K+S+B)$ численно эффективен и объемен над $Z$. Следовательно, естественное ограничение

$$
H^{0}\left(Y,\left\lceil\mathbb{M}_{Y}+\mathbb{A}_{Y}^{\prime}\right\rceil\right) \rightarrow H^{0}\left(S^{\prime},\left\lceil\mathbb{M}_{H_{S^{\prime}}}+\mathbb{A}_{S^{\prime}}^{0}\right\rceil\right)
$$

сюръективно. 
Так как Mov $\left\lceil\mathbb{M}_{Y}+\mathbb{A}_{Y}^{\prime}\right\rceil \leqslant \mathbb{M}_{Y}$, то по сюръективности Mov $\left\lceil\mathbb{M}_{H_{\mid S^{\prime}}}+\mathbb{A}_{S^{\prime}}^{0}\right\rceil$ не содержит ничего, кроме ограничения $\operatorname{Mov} \mathbb{M}_{H_{S^{\prime}}}$. Это доказывает насышение на $S^{\prime} \rightarrow S$.

Насьшение на последуюших раздутиях $S^{\prime \prime} \rightarrow S^{\prime} \rightarrow S$ следует из леммы 5.13.

Теперь рассмотрим некоторые свойства pbd-алгебр, как функциональных алгебр, возникаюших из полуаддитивной (вверх) последовательности $\mathbb{M}_{*}=\left\{\mathbb{M}_{i}\right\}, 0<i \in \mathbb{N}$, цельх подвижных $\mathrm{b}$-дивизоров $\mathbb{M}_{i}, i \in \mathbb{N}$, многообразия $X$. Полуаддитивность означает, что $\mathbb{M}_{1} \geqslant 0$ и $\mathbb{M}_{i}+\mathbb{M}_{j} \leqslant \mathbb{M}_{i+j}$ для всех $i, j \in \mathbb{N}$. С такой последовательностью связана ее характеристическая выпуклая (вверх) последовательность $\mathbb{Q}$-b-дивизоров $\mathbb{D}_{*}=\left\{\mathbb{D}_{i}\right\}, 0<i \in \mathbb{N}$,

$$
\mathbb{D}_{i}=\frac{1}{i} \mathbb{M}_{i}
$$

Вьпуклость означает, что $\mathbb{D}_{1} \geqslant 0$ и $i \mathbb{D}_{i}+j \mathbb{D}_{j} \leqslant(i+j) \mathbb{D}_{i+j}$ для всех $i, j \in \mathbb{N}$. Будем говорить, что последовательность $\mathbb{D}_{*}$ ограничена (сверху), если существует b-дивизор $\mathbb{D}$ с $\mathbb{D}_{i} \leqslant \mathbb{D}$ для всех $i \in N$.

Вьпуклая последовательность является возрастаюшей в (мультипликативном) смысле, т.е. $\mathbb{D}_{i} \leqslant \mathbb{D}_{j}$, когда $i$ делит $j$. Если $\mathbb{D}_{*}$ ограничена, то из вьпуклости следует, что сушествует предел как $\mathbb{R}$-b-дивизор

$$
\mathbb{D}=\lim _{i \rightarrow \infty} \mathbb{D}_{i}=\sup \mathbb{D}_{i} \in \operatorname{Div} X \otimes \mathbb{R}
$$

Если мы имеем полуаддитивную последовательность (подвижных) b-дивизоров $\mathbb{M}=\left\{\mathbb{M}_{i}\right\}$, то при переходе к пределу предпочтительнее работать с ее характеристической выпуклой последовательностью $\mathbb{D}_{*}=\left\{\mathbb{D}_{i}\right\}$, где $i \mathbb{D}_{i}=\mathbb{M}_{i}$.

ОПРЕДЕЛЕНИЕ 5.19. pbd-алгеброй называется функциональная алгебра $R=$ $R\left(X, \mathbb{D}_{*}\right)$, ассоциированная с вьпуклой последовательностью $\mathbb{R}$-b-дивизоров $\mathbb{D}_{*}$. По определению

$$
R_{i}=H^{0}\left(X, i \mathbb{D}_{i}\right)=H^{0}\left(X, \mathscr{O}_{X}\left(i \mathbb{D}_{i}\right)\right) \text { для всех } i \geqslant 1 \text { и } R_{0}=A=H^{0}\left(Z, \mathscr{O}_{Z}\right)
$$

на аффиннном многообразии $Z$, как вьше. По определению пучков $\mathscr{O}_{X}\left(i \mathbb{D}_{i}\right)$ имеем включения $R_{i} \subset k(X)$, а по вьпуклости $\mathbb{D}_{*}-$ включения $R_{i} R_{j} \subset R_{i+j}$, где умножение задается как произведение функций. Мы предполагаем также, что последовательность $\left\{\mathbb{D}_{i}\right\}$ такова, что вьполнено условие конечной порожденности (когерентности) для каждого $A$-модуля $R_{i}$ над $A$. Таким образом, pbd-алгебра является функциональной алгеброй и $\mathbb{D}_{*}$ называется ее характеристической последовательностью, если все $i \mathbb{D}_{i}$ подвижны. Алгебра ограничена, если и только если ограничена ее характеристическая последовательность.

Аналогично, pbd-алгебру можно определить для полуаддитивной последовательности $\mathbb{M}_{*}$ подвижных b-дивизоров, полагая $R_{i}=H^{0}\left(X, \mathbb{M}_{i}\right), R_{0}=A$. В таком случае $\mathbb{M}_{*}$ назьвается подвижной последовательностью этой pbd-алгебры. Приставка псевдо добавляется для того, чтобы отличать pbd-алгебры от просто b-дивизориальных алгебр вида

$$
\bigoplus_{m \geqslant 0} H^{0}(X, m \mathbb{D})
$$


связанных с одним b-дивизором $\mathbb{D}$.

Для сдутия $X \rightarrow Z$ на нормальное аффиинное многообразие $Z$ имеем $R\left(X, \mathbb{D}_{*}\right)=$ $R\left(Z, \mathbb{D}_{*}\right)$, так что в некоторых ситуациях мы можем работать с $Z$ вместо $X$, т.е. предполагать $X$ аффинньм, но, возможно, не $\mathbb{Q}$-факториальньм.

ЛЕмма 5.20 [37; 4.15], [7; 3.6.7]. Пусть $X \rightarrow Z-$ сдутие на аффинное многообразие $Z$ и $R=R\left(X, \mathbb{D}_{*}\right)$ - pbd-алгебра на $X / Z$. Тогда существует полуаддитивная последовательность $\mathbb{M}_{*}$ подвижных $b$-дивизоров многообразия $X$ такая, что $R=R\left(X, \mathbb{M}_{*}\right)$. Иначе говоря, всякая $\mathrm{pbd-алгебра} \mathrm{возникает} \mathrm{из} \mathrm{подвижной}$ последовательности, а потому - и характеристической.

ДокаЗАтЕ ЛЬСтво. Рассматривая $Z$ вместо $X$, можно считать $Z=X$ аффинным. Пусть $V_{i}=H^{0}\left(X, i \mathbb{D}_{i}\right) \subset H^{0}\left(X, i \mathbb{D}_{i X}\right)$ - $A$-подмодуль конечного типа. Возьмем b-подвижную часть дивизора $i \mathbb{D}_{i X}$ относительно $V_{i}$ :

$$
\mathbb{M}_{i}=\operatorname{Mov}_{V_{i}} i \mathbb{D}_{i X}
$$

Ясно, что $H^{0}\left(X, \mathbb{M}_{i}\right)=H^{0}\left(X, i \mathbb{D}_{i}\right)$.

ЛЕмма 5.21 (предельньй критерий). Пусть $X \rightarrow Z-$ сдутие на аффинное многообразие $Z$. pbd-алгебра $R=R\left(X, \mathbb{D}_{*}\right)$, заданная характеристической последовательностью $\mathbb{D}_{*}$, конечно порождена, если и только если существует натуральное $m>0$ такое, что $\mathbb{D}_{m i}=\mathbb{D}_{m}$ для всех $i$.

По предыдущей лемме каждая pbd-алгебра может быть задана характеристической последовательностью b-дивизоров $\mathbb{D}_{i}=\mathbb{M}_{i} / i$.

ДоказАТЕльство. Предположим, что $\mathbb{D}_{m i}=\mathbb{D}_{i}$ для всех $i$. Покажем, что $R$ конечно порождена. Переходя к прореженной подалгебре, будем считать $m=1$. Тогда

$$
R=R\left(X, \mathbb{M}_{*}\right)=\bigoplus_{i \geqslant 0} H^{0}(X, i \mathbb{M})
$$

- обычная b-дивизориальная алгебра, ассоциированная с подвижным b-дивизором $\mathbb{M}=\mathbb{D}_{1}$. Пусть $Y \rightarrow X-$ модель, на которой $\mathbb{M}_{Y}$ свободен и $\mathbb{M}=\overline{\mathbb{M}}_{Y}$. Тогда $R=$ $R\left(Y, \mathbb{M}_{Y}\right)$ - дивизориальная алгебра, ассоциированная со свободным дивизором $\mathbb{M}_{Y}$. Хорошо известно, что такие алгебры конечно порождены. Обратно, предположим, что $R$ конечно порождена. С точностью до прореживания можно считать, что она порождена функциями степени 1 , отвечаюшими подвижному b-дивизору $\mathbb{M}_{1}=\mathbb{D}_{1}$. Тогда

$$
\operatorname{Proj} R=\operatorname{Proj} \bigoplus_{i \geqslant 0} H^{0}\left(X, i \mathbb{M}_{1}\right) \text {, }
$$

т.е. подвижная последовательность для $R$ b-дивизориальна. Отсюда следует, что для любого натурального $m>0$ имеем $\mathbb{M}_{m i}=i \mathbb{M}_{m}$ для всех $i$. Переходя к характеристической последовательности, получаем требуемый результат (см. [37; 4.16, 4.28]). 
Лемма $5.22[37 ; 4.15],[7 ; 3.8 .2]$. Пусть $V=\bigoplus_{i \geqslant 0} V_{i}-$ функииональная алгебра на $X / Z$. Существует pbd-алгебра $V \subset R=R\left(X, \mathbb{M}_{*}\right)$, которая целозамкнута в $k(X) / Z u$

a) $V$ ограничена $\Longleftrightarrow R$ ограничена;

b) $V$ конечно порождена $\Longleftrightarrow R$ конечно порождена.

ДокАЗАтельство. Работая с $Z$ вместо $X$ и отождествляя $Z$ с $X$, можно предполагать многообразие $X$ аффинным. По определению функциональной алгебры $V_{i} \subset k(X)$ - конечно порожденный $A$-подмодуль (дробный идеал) для каждого $i$, где $A=H^{0}\left(X, \mathscr{O}_{X}\right)$ - координатное кольцо многообразия $X$. Ясно, что $V_{i} \subset H^{0}\left(X, \mathbb{M}_{i}\right)$ для подвижного b-дивизора $\mathbb{M}_{i}$ (cp. с леммой 5.20), определяемого формулой:

$$
\operatorname{mult}_{E} \mathbb{M}_{i}=-\min _{\varphi \in V_{i}} \operatorname{mult}_{E} \varphi
$$

для всех простых b-дивизоров $E$. Так как $V_{i} V_{j} \subset V_{i+j}$, то последовательность $\mathbb{M}_{*}=$ $\left\{\mathbb{M}_{i}\right\}$ полуаддитивна. Положим $R=R\left(X, \mathbb{M}_{*}\right)$.

Если алгебра $V$ ограничена дивизором $D$, то $R$ ограничена этим же дивизором. Обратное также, очевидно, верно.

Проверим утверждение b). Конечная порожденность влечет ограниченность. Значит, при конечной порожденности одной из алгебр обе ограничены. Умножая на подходящую рациональную функцию, можно предполагать, что все $V_{i} \subset A \subset k(X)$ как идеалы в $A$. Тогда $R_{i} \subset k(X)$ состоит из функций $\varphi$, удовлетворяюших уравнению целого замькания:

$$
x^{n}+a_{1} x^{n-1}+\cdots+a_{n}=0,
$$

где $a_{j} \in V_{i}^{j}$ (индекс вверху означает степень идеала $V_{i}$ ). Так что алгебра $R$ цела над $V$. Алгебры $V$ и $R$ имеют общее поле частных $-k(X)$. Следовательно, по конечности целого замькания $R$ конечна как $V$-модуль.

Если алгебра $V$ конечно порождена, то и $R$ конечно порождена как конечный модуль над $V$. Обратно, предположим, что алгебра $R$ конечно порождена. С точностью до прореживания можно считать, что $R$ порождается $R_{1}$. Для каждого $i$ прореженная подалгебра $R^{(i)} \subset R$ цела над конечно порожденной алгеброй $\bigoplus_{j \geqslant 0} V_{i}^{j}$. Это дает конечные сюръективные морфизмы

$$
Y=\operatorname{Proj} R=\operatorname{Proj} R^{(i)} \rightarrow Y_{i}=\operatorname{Proj} \bigoplus_{j \geqslant 0} V_{i}^{j} .
$$

Существует их естественное разложение $Y \rightarrow Y_{m i} \rightarrow Y_{i}$ в композицию, определяемое вложением $V_{i}^{m j} \subset V_{m i}^{j}$. Для некоторого $i_{0}$ имеем $Y_{m i_{0}}=Y_{i_{0}}$ для всех $m \geqslant 1$. Опять с точностью до прореживания можно считать, что $i_{0}=1$. Тогда $Y_{m}=Y_{1}$ означает, что для всех $m$ вьполнены равенства $V_{m}^{j}=V_{1}^{j m}$ при некоторых $j \gg 1$. Как и выше, считаем, что все $V_{m} \subset A$-идеалы. Тогда ясно, что $A$-модуль $V_{m}$ цел над $A$-модулем $V_{1}^{m}$ и, следовательно, алгебра $V$ цела над $\bigoplus_{m \geqslant 0} V_{1}^{m}$, а потому конечна над последней алгеброй. Стало быть, и $V$ конечно порождена.

Обсудим, наконец, наиболее важный момент теории - гипотезу о конечной порожденности некоторых pbd-алгебр. Отметим сразу одно из очевидных необходимых условий - pbd-алгебра $R$ должна быть ограниченной.

Но наиболее тонкое и существенное условие - это асимптотическое насыщение, введенное впервые в $[37 ; 4.33]$. 
ОПРЕДЕЛЕНИЕ 5.23. Вьпуклая последовательность эффективных b-дивизоров $\mathbb{D}_{*}=\left\{\mathbb{D}_{i}\right\}$ называется асимптотически $\mathbb{C}$-насьщенной, если (с точностью до прореживания) для всех натуральных $i, j$ :

$$
\operatorname{Mov}\left\lceil j \mathbb{D}_{i Y}+\mathbb{C}_{Y}\right\rceil \leqslant j \mathbb{D}_{j Y}
$$

на всех достаточно раздутых моделях $Y \rightarrow X$. Это означает, что (после прореживания) для каждой пары $(i, j)$ сушествует модель $Y_{(i, j)} \rightarrow X$ такая, что неравенство (5.1) выполнено на всех моделях $Y \rightarrow Y_{(i, j)} \rightarrow X$.

$\mathrm{B}$ случае, когда $\mathbb{C}=\mathbb{A}=\mathbb{A}(X, B)$ - b-дивизор дискрепантностей логпары $(X, B)$, будем говорить, что эта последовательность логканонически асимптотически (lca-) насыщена над $(X, B)$. pbd-алгебра $R$ назьвается lса-насыщенной, если ее характеристическая последовательность такова.

Практически для использования асимптотического насьщения необходимо найти модель $Y$, не зависящую от $i, j$, где асимптотическое насьшение выполнено равномерно. Существование такой модели далеко не тривиально - это CCS-гипотеза в [37; 6.14], и мы можем построить ее пока только в случае поверхностей (см. следующий $\S 6$, в частности, следствие 6.6).

Смысл асимптотического насьшения в том, что неравенство (5.1) становится сильнее при $i \rightarrow \infty$. В случае равномерного асимптотического насьшения обычно используется такое его следствие: пусть $\mathbb{D}=\lim _{i \rightarrow \infty} \mathbb{D}_{i}=\sup \left\{\mathbb{D}_{i}\right\}$ и насьшение вьполнено на $Y$ для всех $i, j$, тогда

$$
\operatorname{Mov}\left\lceil j \mathbb{D}_{Y}+\mathbb{C}_{Y}\right\rceil \leqslant j \mathbb{D}_{j Y} \leqslant j \mathbb{D}_{Y} \text { для всех } j \text {. }
$$

Это позволяет в определенной ситуации доказать, что предельньй b-дивизор рационален (см. следующий $\S 6$, в частности, следствие 6.12$)$.

ОПРЕДЕЛЕНИЕ 5.24. Пусть $(X, B)$ - klt пара и $X \rightarrow Z$ - ее слабое сдутие Фано на аффинное многообразие $Z$. Ограниченная lса-насьшенная pbd-алгебра на $X / Z$ называется FGA-алгеброй.

ГИПотЕЗА 5.25 (о конечной порожденности). Всякая FGA-алгебра конечно порождена.

\section{§. Конечная порожденность FGA-алгебр в размерности 2}

6.1. В этом параграфе мы покажем, что FGA-гипотеза 5.25 влечет существование допредельного флипа, и в конще докажем эту гипотезу для поверхности.

Пусть $\varphi:(X, S+B) \rightarrow Y$ - допредельное сдутие, в частности, дивизор $K+S+B$ plt и $\varphi$-антиобилен. Выберем $\varphi$-антиобильньй дивизор $M$ на $X$, носитель которого не содержит $S$. Допредельньй флип сушествует, если алгебра

$$
\mathscr{R}=\mathscr{R}_{X / Z} M=\bigoplus_{i \geqslant 0} H^{0}(X, i M)
$$

конечно порождена или, эквивалентно, существует $M$-фрлип. Ограничим еена $S: R^{0}=$ $\operatorname{res}_{S} \mathscr{R}$. По определению $R^{0}=\bigoplus R_{i}^{0}$, где $R_{i}^{0}=\operatorname{Im}\left(H^{0}(X, i M) \rightarrow H^{0}\left(X, i M_{\mid S}\right)\right)$. 
Мы доказали в предложении 5.4, что $M$-флип сушествует, если вычетная алгебра $R^{0}$ конечно порождена. По лемме 5.22 целое замыкание алгебры $R^{0}$ в $k(S) / Z$ является pbd-алгеброй $R_{S}$ и $R^{0}$ конечно порождена, если и только если алгебра $R_{S}$ конечно порождена. По доказательству леммы $5.22 R_{S}=R\left(S, \mathbb{M}_{*}^{0}\right)$, где

$$
\mathbb{M}_{i}^{0}=\operatorname{res}_{S} \mathbb{M o v} i M
$$

является подвижным ограничением b-подвижной части дивизора $i M$, как в определении 5.15 (ii). По ограниченности дивизориальной алгебры $\mathscr{R}$ и снова по лемме 5.22 pbd-алгебра $R_{S}$ ограничена.

ЛЕмма $6.2[37 ; 4.50 .1],[7 ; 4.1 .1] . R_{S}$ является FGA-алгеброй.

ДокАЗАТЕЛЬСтво. Нетрудно проверить, что $\left(S, B_{S}\right) \rightarrow Z$ - тоже слабое сдутие Фано. Итак, нужно показать, что алгебра $R_{S}$ lса-насышена. Отметим, что lса-насыщение - инвариант целого замыкания, поскольку подвижная часть соответствующих линейных систем не меняется при целом замыкании, а меняться может лишш их размерность. По лемме 5.16 каждый b-дивизор Mov $i M$ исключительно насышен. Следовательно, применима лемма 5.18. Из нее получаем, что все $\mathbb{M}_{i}^{0} \mathbb{A}\left(S, B_{S}\right)$-насьшены. Чтобы доказать lса-насьшение, надо вернуться к доказательству леммы 5.18 и сделать необходимые изменения.

Для простоты обозначений положим $\mathbb{M}_{i}=\mathbb{M o v} i M$. По конструкции и потому, что $M$ - дивизор Картье, имеем

$$
\mathbb{M}_{i Y}=\operatorname{Mov} f^{*} i M
$$

на всех моделях $f: Y \rightarrow X$. По предыдушему $\mathbb{M}_{i}^{0}=\operatorname{res}_{S} \mathbb{M}_{i}$ (подвижное ограничение).

Для проверки lса-насьшения фиксируем пару индексов $(i, j)$, и пусть $f: Y \rightarrow X-$ логразрешение пары $\left(X, S+B+\mathbb{D}_{i X}\right), F_{m}-f$-исключительные простые дивизоры, а $D_{l}^{\prime}$ и $S^{\prime}$ - собственные прообразы простых компонент $B$ и $S$. Предположим, что $Y \rightarrow X$ - достаточно раздутая модель, так что

a) след $\mathbb{M}_{i Y}$ свободен и $\mathbb{M}_{i}=\overline{\mathbb{M}_{i Y}}$;

b) то же самое справедливо для $\mathbb{M}_{j}$.

Модель $Y \rightarrow X$ зависит, вообше говоря, от $i, j$. Запишем

$$
K_{Y}=f^{*}(K+S+B)-S^{\prime}-\sum b_{l} D_{l}^{\prime}+\sum a_{m} F_{m}
$$

на $Y$, где по определению $\mathbb{A}_{Y}=-S^{\prime}-\sum b_{l} D_{l}^{\prime}+\sum a_{m} F_{m}$. Мы хотим показать, что pbd-алгебра $R_{S}=R\left(S, \mathbb{M}_{*}^{0}\right)$ lса-насышена для выбранных $i, j$. Это свойство характеристической последовательности, так что перейдем к $\mathbb{Q}$-b-дивизорам $\mathbb{D}_{i}=\frac{1}{i} \mathbb{M}_{i}$. Сначала проверим, что свойство насьшения вьполнено для $j \mathbb{D}_{i}$ и $j \mathbb{D}_{j}$ на модели $S^{\prime} \rightarrow S$. Для простоты обозначений положим $\mathbb{A}^{0}=\mathbb{A}\left(S, B_{S}\right)$. Надо сравнить $\operatorname{Mov}\left\lceil\left. j \mathbb{D}_{i Y}\right|_{S^{\prime}}+\right.$ $\left.\mathbb{A}_{S^{\prime}}^{0}\right\rceil$ с $\left.j \mathbb{D}_{j Y}\right|_{S^{\prime}}$. Заметим, что по формуле присоединения $\mathbb{A}_{S^{\prime}}^{0}=\left.\mathbb{A}_{Y}^{\prime}\right|_{S^{\prime}}$, где $\mathbb{A}^{\prime}=\mathbb{A}+\underline{S}$.

По теореме обрашения в нуль Каваматы-Фивега

$$
H^{1}\left(Y,\left\lceil\left(j \mathbb{D}_{i}+\mathbb{A}\right)_{Y}\right\rceil\right)=0
$$


так как $-(K+S+B)$ численно эффективен и объемен над $Z$. Следовательно, отображение ограничения

$$
H^{0}\left(Y,\left\lceil\left(j \mathbb{D}_{i}+\mathbb{A}^{\prime}\right)_{Y}\right\rceil\right) \rightarrow H^{0}\left(S^{\prime},\left\lceil\left(j \mathbb{D}_{i}^{0}+\mathbb{A}^{0}\right)_{S^{\prime}}\right\rceil\right)
$$

где $\mathbb{D}_{i}^{0}=\frac{1}{i} \mathbb{M}_{i}^{0}=\operatorname{res}_{S} \mathbb{D}_{i}$, сюръективно. Взятие целой части перестановочно с ограничением по выбору логразрешения. Для доказательства утверждения достаточно показать, что

$$
\operatorname{Mov}\left\lceil\left(j \mathbb{D}_{i}+\mathbb{A}^{\prime}\right)_{Y}\right\rceil \leqslant j \mathbb{D}_{j Y}=\mathbb{M}_{j Y} .
$$

Как уже отмечалось, $\mathbb{M}_{i Y}=\operatorname{Mov} f^{*} i M$. Следовательно, то, что мы хотим доказать, эквивалентно неравенству

$$
\operatorname{Mov}\left\lceil\frac{j}{i} \operatorname{Mov} f^{*} i M+\mathbb{A}_{Y}^{\prime}\right\rceil \leqslant \operatorname{Mov} f^{*} j M .
$$

Последнее совсем просто, поскольку дивизор

$$
f_{*} \operatorname{Mov}\left\lceil\frac{j}{i} \operatorname{Mov} f^{*} i M+\mathbb{A}_{Y}^{\prime}\right\rceil=j M=f_{*} \operatorname{Mov} f^{*} j M
$$

цел и положительная часть дивизора $\mathbb{A}_{Y}^{\prime}$ исключительна. Поэтому последнее неравенство следует из исключительного насышения $\mathbb{M o v} j M$ леммы 5.16.

Теперь по лемме 5.13 свойство асимптотического насьшения для $i, j$ вьполнено на всех моделях $S^{\prime \prime} \rightarrow S^{\prime} \rightarrow S$. Это завершает доказательство.

Теперь будем работать с поверхностями. Пусть $(X, B)$ - 2-мерная klt пара и $X \rightarrow Z$ - слабое сдутие $\Phi$ ано, а $R=R\left(X, \mathbb{D}_{*}\right)$ - FGA-алгебра с характеристической последовательностью $\mathbb{D}_{*}=\left\{\mathbb{D}_{i}\right\}$. Мы хотим доказать, что $R$ конечно порождена. Первая значительная трудность - это найти модель $Y \rightarrow X$, на которой все асимптотические насьшения

$$
\operatorname{Mov}\left\lceil j \mathbb{D}_{i Y}+\mathbb{A}_{Y}\right\rceil \leqslant j \mathbb{D}_{j Y}
$$

вьполнены равномерно независимо от $i, j$. Чуть ниже мы покажем, что такой моделью является так назьваемая крепантная терминальная модель $\psi:\left(X^{\prime}, B^{\prime}\right) \rightarrow(X, B)$, т.е. $\left(X^{\prime}, B^{\prime}\right)-\operatorname{trm}$ пара и $K_{X^{\prime}}+B^{\prime}=\psi^{*}(K+B)$.

ОПРЕДЕЛЕНИЕ 6.3 . Пусть $\mathbb{D}$ - b-дивизор многообразия $X$. Будем говорить, что $\mathbb{D}$ спускается на $X$, если $\mathbb{D}=\overline{\mathbb{D}_{X}}$.

Случай поверхностей является очень специальным, потому что подвижные дивизоры на (неособой) поверхности являются численно эффективными. Это - главньй момент в доказательстве следующего основного результата этого параграффа.

Теорема 6.4. Пусть $(X, B)$ - 2-мерная trm nара. Это означает, что $X$ неособо и $0 \leqslant B=\sum b_{i} D_{i}$ - эффективныи $\mathbb{R}$-дивизор на $X$ такой, что mult $_{x} B<1$ в каждой (замкнутой) точке $x \in X$. Пусть $X \rightarrow Z$ - слабое сдутие Фано на аффинную поверхность $Z$. Если $\mathbb{M}$ - подвижсный $\mathbb{A}$-насыщенный $b$-дивизор многообразия $X$, где $\mathbb{A}=\mathbb{A}(X, B)$, то $\mathbb{M}$ спускается на $X$. 
ДокАЗАТЕЛЬСТвО. Пусть $f: Y \rightarrow X$ - достаточное сильное логразрешение пары $(X, B)$ такое, что $\mathbb{M}=\overline{\mathbb{M}}_{Y}$ и, следовательно, линейная система

$$
\left|\mathbb{M}_{Y}\right|=|\mathbb{M}|=\left\{\operatorname{div}(\varphi)+\mathbb{M} \mid 0 \neq \varphi \in H^{0}(X, \mathbb{M})\right\}
$$

свободна.

Утверждение: дивизор $E=\left\lceil\mathbb{A}_{Y}\right\rceil$ - цельй, $f$-исключительньй и

(i) каждый (эффективный) $f$-исключительный дивизор сосредоточен в носителе $E$ (с положительными кратностями), т.е. Supp $E$ состоит из всех исключительных простых дивизоров;

(ii) $H^{1}(Y, E)=0$.

Для доказательства этого утверждения запишем

$$
K_{Y}=f^{*}(K+B)-B_{Y}+\sum a_{i} E_{i},
$$

где дискрепантность $\mathbb{A}_{Y}=-B_{Y}+\sum a_{i} E_{i}$ и все исключительные кратности $a_{i}>0$. В частности, для $E=\left\lceil\mathbb{A}_{Y}\right\rceil$ выполнено свойство (i). Заметим, что $\mathbb{R}$-дивизор

$$
-f^{*}(K+B)=-K_{Y}+\mathbb{A}_{Y}
$$

численно эффективен и объемен над $Z$, так что по теореме обращения в нуль Каваматы-Фивега $H^{1}\left(Y,\left\lceil\mathbb{A}_{Y}\right\rceil\right)=H^{1}(Y, E)=0$. Это доказывает (ii).

По предположению $\mathrm{b}$-дивизор $\mathbb{M} \mathbb{A}$-насьщен. Это означает, что

$$
E=\operatorname{Fix}\left(\mathbb{M}_{Y}+E\right) .
$$

Пусть $M_{Y} \in\left|\mathbb{M}_{Y}\right|$ - обший элемент. Можно предполагать, что это - кривая, ибо в противном случае $\mathbb{M}=0=\overline{0_{X}}$. Тогда по обрашению в нуль (ii) отображение ограничения

$$
H^{0}\left(Y, \mathbb{M}_{Y}+E\right) \rightarrow H^{0}\left(M_{Y},\left(\mathbb{M}_{Y}+E\right)_{\left.\right|_{Y}}\right)
$$

сюръективно. Следовательно,

$$
E_{\mid M_{Y}}=\operatorname{Fix}\left(\left(\mathbb{M}_{Y}+E\right)_{\left.\right|_{Y}}\right) .
$$

Но $M_{Y}$ - аффинная кривая. Поэтому любая полная линейная система на $M_{Y}$ свободна. По сюръективности отображения ограничения система $\left|\mathbb{M}_{Y}+E\right|$ свободна на $Y$ вблизи $M_{Y}$. Значит, $\operatorname{Supp} E \cap M_{Y}=\varnothing$. Так как носитель $E$ содержит все исключительные дивизоры, это означает, что $M_{Y}$ не пересекает ни одну из исключительных кривых, т.е. $\mathbb{M}_{Y}=f^{*} \mathbb{M}_{X}$ и $\mathbb{M}=\overline{\mathbb{M}_{X}}$.

Напомним следуюший хорошо известньй результат о сушествовании крепантного терминального раздутия. 
УСЛОВНАЯ ТЕОРЕМА 6.5 (в предположении, что логПММ выполнена в размерности $\operatorname{dim} X[36 ; 3.1])$. Пусть $(X, B)$ - klt пара. Тогда существует проективное $\left(\mathbb{Q}\right.$-факториальное) раздутие $\psi: X^{\prime} \rightarrow X$ такое, что

(i) $\left(X^{\prime}, B^{\prime}\right)-\operatorname{trm}$ napa;

(ii) $K_{X^{\prime}}+B^{\prime}=\psi^{*}(K+B)$, m.е. $\left(X^{\prime}, B^{\prime}\right)$ - крепантная пара над $(X, B)$.

Пара $\left(X^{\prime}, B^{\prime}\right)$ называется крепантною терминальной моделью (см. определение 1.6 (iii) и ср. с определением 1.9 (ii) пары $(X, B))$.

При $\operatorname{dim} X \leqslant 4$ предполохсение о логПММ можсно опустить. B случае поверхностей крепантная терминальная модель существует и единственна.

Прежде чем доказывать теорему, выведем следующее следствие.

СЛЕДСТВИЕ 6.6. Пусть $(X, B)$ - klt логповерхность, $X \rightarrow Z$ - слабое сдутие Фано и $R=R\left(X, \mathbb{M}_{*}\right)$ - FGA-алгебра с подвижной последовательностью $\mathbb{M}_{*}=$ $\left\{\mathbb{M}_{i}\right\}$. Если $\left(X^{\prime}, B^{\prime}\right) \rightarrow(X, B)$ - крепантная терминальная модель, то $X^{\prime} \rightarrow Z$ - тоже слабое сдутие Фано и все $\mathbb{M}_{i}$ спускаются на $X^{\prime}$. Пусть $G=\sum G_{l}-$ дивизор на $X^{\prime}$, содержащий носители всех $\mathbb{M}_{i X^{\prime}}$, и $X^{\prime \prime} \rightarrow X^{\prime}$ - логразрешение для $\left(X^{\prime}, B^{\prime}+G\right)$. Тогда асимптотическое $\mathbb{A}$-насыщение выполнено равномерно на моделях $Y \rightarrow X^{\prime \prime}$, раздуваемых из $X^{\prime \prime}$, т.е. на всех таких моделях

$$
\operatorname{Mov}\left\lceil j \mathbb{D}_{i Y}+\mathbb{A}_{Y}\right\rceil \leqslant j \mathbb{D}_{j Y} .
$$

ДокАЗАТЕЛЬСТВО. Это вытекает из леммы 5.13. Отметим также, что асимптотическое $\mathbb{A}$-насыщение при $i=j$ влечет $\mathbb{A}$-насьпение b-дивизора $\mathbb{M}_{i}=i \mathbb{D}_{i}$.

СлЕДСТВИЕ 6.7. В предположениях теоремы 6.5 многообразие (пространство, схема) $X$ имеет проективную $\mathbb{Q}$-факториализацию (локально над компактнымм, полнымм подмножеством в $X$ ), т.е. малое раздутие $X^{\prime} \rightarrow X$ (соответственно аналитическое, формальное) такое, что $X^{\prime} \mathbb{Q}$-факториально (соответственно локально над компактным, полным подмножеством в $X$ ).

$\Pi р и \operatorname{dim} X \leqslant 4$ предположение о логПММ моэсно опустить, а klt свойство ослабить до lt свойства пары $(X, B)$ при наличии проективного разрешения в определении lt.

НАБРОСОК ДОКАЗАТЕЛЬСТВА ТЕОРЕМЫ 6.5 И СЛЕДСТВИЯ 6.7 . Результаты хорошо известны при $\operatorname{dim} X \leqslant 3$, и конструкция для их доказательства восходит, по-видимому, к [11]. Пусть $Y \rightarrow X-$ (соответственно аналитическое, формальное) относительно проективное логразрешение пары $(X, B)$ (соответственно локально над компактньм, полньм подмножеством в $X$ ) с исключительными простыми дивизорами $E_{i}$, включаюшими все с дискрепантностями $\leqslant 0$. Пусть $\left(X^{\prime}, B^{\prime}\right)$ - логпара, полученная применением логПММ к $\left(Y, B_{Y}+\sum b_{i} E_{i}\right)$ над $X$ (соответственно локально над компактньм, полным подмножеством в $X)$, где в $\sum$ берутся лишш исключительные $E_{i}$ с $b_{i}=-a\left(E_{i}, X, B\right) \geqslant 0$. Тогда утверждение теоремы вытекает из следуюших замечаний:

a) $\left(X^{\prime}, B^{\prime}\right)$ - строго минимальная и одновременно логминимальная модель пары $\left(Y, B_{Y}+\sum b_{i} E_{i}\right)$, которая не сдувает компонент границы $B_{Y}$ и исключительных простых дивизоров $E_{i}$ с дискрепантностями $a\left(E_{i}, X, B\right) \leqslant 0$; 
b) $\left(X^{\prime}, B^{\prime}\right)$ - крепантное раздутие $(X, B)$ в точности всех исключительных простых дивизоров с дискрепантностями $\leqslant 0$;

c) $\left(X^{\prime}, B^{\prime}\right)$ - строго терминальная пара.

Из неравенства, с точностью до уменьшения кратностей гранишы, леммы 2.4 или дивизориальной версии вместе с флипами в монотонности 3.4 (i) следует а), поскольку $(X, B)$ - логканоническая модель пар $\left(Y, B_{Y}+\sum b_{i} E_{i}\right)$ и $\left(X^{\prime}, B^{\prime}\right)$. По тем же соображениям получаем b) (cp. с [34; 1.5 .7$]$ ). По конструкции логтерминальных пар получаем с). Для логповерхностей конструкция есть не что иное, как сдутие на логразрешении всех (-1)-кривых с положительными дискрепантностями.

По [37; 1.8$]$ и [39; Example 9] логПММ можно опустить для $\operatorname{dim} X \leqslant 4$, так как исходную модель $\left(Y, B_{Y}+\sum b_{i} E_{i}\right)$ и все последуюшие можно предполагать $t r m$.

Отметим, что если $(X, B)$ - trm пара, то конструкция дает малое раздутие - требуемую $\mathbb{Q}$-факториализацию. Чтобы получить подобную $\mathbb{Q}$-факториализацию при klt особенностях, надо в качестве исходной пары взять $\left(Y, B_{Y}^{\log }\right)$, т.е. поменять все исключительные кратности $b_{i}$ на 1 . При этом, увеличивая границу, можно обойтись специальньм обрьвом теоремы 4.8 вместо обшего (см. доказательство [36; 3.1$]$ и редукцию теоремы 4.7) и специальный обрыв выполнен при $\operatorname{dim} X \leqslant 4$ по следствию 4.11.

Докажем теперь гипотезу 5.25 на поверхностях. Из этого, как мы уже знаем, следует существование 3 -мерных флипов.

Теорема 6.8. Пусть $(X, B)$ - 2-мерная klt пара, а $\varphi: X \rightarrow Z$ - ее слабое сдутие Фано на аффинную поверхность $Z$ (т.е. $\varphi_{*} \mathscr{O}_{X}=\mathscr{O}_{Z}$ и дивизор $-(K+B)$ численно әффективен над $Z$, его облемность над $Z$ вытекает из бирациональности $\varphi$ ). Тогда всякая FGA-алгебра на $X / Z$ конечно порождена.

Оставшаяся часть параграф̆а посвяшена доказательству этой теоремы. Многие утверждения верны во всех размерностях. Будут отмечены места, где используется то, что $X-$ поверхность.

ДокАЗАТЕЛьСтво. Шаг 1: обозначения и предварительные замечания. Пусть $R=R\left(X, \mathbb{D}_{*}\right)$ - FGA-алгебра с характеристической последовательностью $\mathbb{D}_{*}=\left\{\mathbb{D}_{i}\right\}$ и подвижной последовательностью $\mathbb{M}_{*}=\left\{\mathbb{M}_{i}\right\}, \mathbb{M}_{i}=i \mathbb{D}_{i}$. Пусть $f:\left(X^{\prime}, B^{\prime}\right) \rightarrow$ $(X, B)$ - крепантная терминальная модель для пары $(X, B)$. Так как $-\left(K_{X^{\prime}}+B^{\prime}\right)=$ $-f^{*}(K+B)$, то сдутие $X^{\prime} \rightarrow Z$ остается еще слабым сдутием Фано. Ясно, что $R=R\left(X^{\prime}, \mathbb{D}_{*}\right)$ - также FGA-алгебра, но на $X^{\prime} / Z$. Следовательно, переходя к крепантной терминальной модели, мы можем предполагать, что $(X, B)$ - trm пара.

Первое из требований для FGA-алгебр - ограниченность. $R$ ограничена, в частности, существует приведенный дивизор $G=\sum G_{j}$ на $X$ такой, что $\operatorname{Supp} \mathbb{D}_{i X} \subset \operatorname{Supp} G$ при всех $i$. Кроме того, по ограниченности последовательность $\mathbb{D}_{*}$ имеет предел

$$
\mathbb{D}=\lim _{i \rightarrow \infty} \mathbb{D}_{i} \in(\operatorname{Div} X) \otimes \mathbb{R}
$$

как b-дивизор, возможно, с вешественными кратностями, и $\operatorname{Supp} \mathbb{D}_{X} \subset G$. Наша цель - показать, что $\mathbb{D}$ есть b-дивизор с рациональными кратностями и что $\mathbb{D}=\mathbb{D}_{m}$ для некоторого $m \gg 1$ и, следовательно, для всех $l$, больших и делимых на $m$.

Шаг 2: полуобильность $\mathbb{D}_{X}$. Здесь мы будем пользоваться тем, что $X$ - поверхность. 
ЛЕмма 6.9. Дивизор $\mathbb{D}_{X}$ полуобилен.

ДокАЗАТЕЛЬСТВо. b-дивизоры $\mathbb{M}_{i}$ подвижны и их следы на поверхности $X$ численно эффективны над $Z$. Следовательно, предел $\mathbb{D}_{X}=\lim _{i \rightarrow \infty} \mathbb{D}_{i X}$ тоже численно эффективен над $Z$. Конус Клеймана-Мори на $X / Z$ полиэдрален, так как $X \rightarrow$ $Z$ - слабое сдутие $\Phi$ ано. Двойственньй конус численно эффективных $\mathbb{R}$-дивизоров порождается полуобильньми дивизорами, опорными для (стягиваемых экстремальных) граней конуса Клемана-Мори. Следовательно, все численно эффективные дивизоры на $X$ полуобильны.

Шаг 3: диофантово приближение. Мы будем работать с целочисленной решеткой $N_{\mathbb{Z}}^{1}=\bigoplus \mathbb{Z} G_{j} \subset \operatorname{Div} X$ и векторньми пространствами $N_{\mathbb{Q}}^{1}=N_{\mathbb{Z}}^{1} \otimes \mathbb{Q}$ и $N_{\mathbb{R}}^{1}=N_{\mathbb{Z}}^{1} \otimes \mathbb{R}$. Поскольку $\mathbb{D}_{X}$ полуобилен, то мы можем выбрать конечное число свободных дивизоров $P_{j} \in N_{\mathbb{Z}}^{1}$, образующих базис $N_{\mathbb{Q}}^{1}$ и таких, что $\mathbb{D}_{X}$ принадлежит конусу

$$
\mathscr{P}=\sum \mathbb{R}_{+} P_{j} \subset \sum \mathbb{R} G_{j} \subset N_{\mathbb{R}}^{1}
$$

порожденному дивизорами $P_{j}$, где $\mathbb{R}_{+}=\{r \in \mathbb{R} \mid r \geqslant 0\}$. Имеет место следующее просто доказьваемое утверждение в стиле диофантовых приближений.

ЛЕмма 6.10. Для каждого $\varepsilon>0$ существуют иелое положительное число m и дивизор $M \in N_{\mathbb{Z}}^{1}$ такие, что

(i) линейная система $|M|$ свободна;

(ii) $\left\|m \mathbb{D}_{X}-M\right\|<\varepsilon$, где норма определяется как максимум модуля координат в базисе $\left\{G_{j}\right\}$ в $N_{\mathbb{R}}^{1} ; u$

(iii) если дивизор $\mathbb{D}_{X}$ не рачионален, то дивизор $m \mathbb{D}_{X}-M$ не әффективен.

ДокАЗАТЕльство. Получается прямо из теоремы Кронекера [3; 1.3$]$. В случае рационального следа $\mathbb{D}_{X}$ берем такое целое $m>0$, что свободен $M=m \mathbb{D}_{X}$. Такое $m$ сушествует по лемме 6.9 .

В случае нерационального $\mathbb{D}_{X}$ будем работать с целочисленной решеткой $N_{\mathbb{Z}}$, порожденной дивизорами $\left\{P_{k}\right\}, k=1, \ldots, l$, и векторными пространствами $N_{\mathbb{Q}}^{1}$ и $\mathbb{N}_{\mathbb{R}}^{1}$ с этим базисом. Так что мы отождествляем их с $\mathbb{Z}^{l}, \mathbb{Q}^{l}$ и $\mathbb{R}^{l}$ соответственно. Запишем $\mathbb{D}_{X}$ в виде вектора $\mathbb{D}_{X}=\mathbf{d}=\left(d_{1}, \ldots, d_{l}\right) \in \mathbb{R}^{l}=\mathbb{N}_{\mathbb{R}}^{1}$, где все $d_{i} \geqslant 0$ вешественны. Так как вектор $\mathbf{d}$ не рационален, то он содержится в минимальном векторном подпространстве в $\mathbb{R}^{l}$ над $\mathbb{Q}$ размерности $\geqslant 1$, которое лежит в подпространстве квадранта, отвечающего положительньм координатам $\mathbf{d}$, и в котором кратности $\mathbf{d}$ сколь угодно близко приближаются узлами решетки $\mathbb{N}_{Z}$. Следовательно, по $[3 ; 1.3]$ для каждого $\varepsilon>0$ можно найти целое положительное кратное $m \mathbf{d}$ вектора $\mathbf{d}$ и цельй вектор $\mathbf{m}=\left(m_{1}, \ldots, m_{l}\right) \in N_{Z}$ такой, что

1) все $m_{i} \geqslant 0$;

2) $\|m \mathbf{d}-\mathbf{m}\|<\varepsilon$

3) дивизор $m \mathbf{d}-\mathbf{m}$ не эффективен (приближение сверху).

Последнего можно добиться в силу свойства строгой вьпуклости множества эффективных дивизоров: оно не содержит прямых.

Положим $M=\sum m_{i} P_{i} \in N_{\mathbb{Z}}=\mathbb{Z}^{l}$. Тогда линейная система $|M|$ свободна по 1$)$. 
Шаг 4: необращение в нуль для линейных систем. Пусть $G$ - (эффективный) дивизор шага 1. Выберем очень малое $\gamma>0$ так, что логпара $(X, B+\gamma G)$ остается еше klt. Если $\mathbb{A}=\mathbb{A}(X, B)$ - b-дивизор дискрепантностей, то klt для $(X, B+\gamma G)$ означает не-

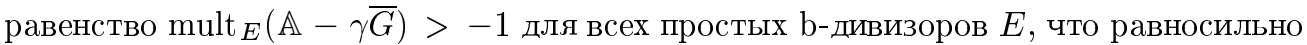
эффективности

$$
\lceil\mathbb{A}-\gamma \bar{G}\rceil \geqslant 0 \text {. }
$$

ЛЕмма 6.11 (о необрашении в нуль). Предположсим, что дивизор $M$ как в лемме 6.10. Тогда если $\varepsilon<\gamma$, то накаждой модели $f: Y \rightarrow X$

$$
\operatorname{Mov}\left\lceil m \mathbb{D}_{Y}+\mathbb{A}_{Y}\right\rceil \geqslant(\bar{M})_{Y}=f^{*} M \text {. }
$$

ДоКАЗАТЕЛЬСТВо. Обозначим $F=m \mathbb{D}_{X}-M$ и запишем

$$
m \mathbb{D}_{Y}+\mathbb{A}_{Y}=f^{*}\left(m \mathbb{D}_{X}\right)+\mathbb{A}_{Y}=f^{*} M+f^{*} F+\mathbb{A}_{Y} \geqslant f^{*} M-\varepsilon f^{*} G+\mathbb{A}_{Y}
$$

Первое равенство - по спуску следствия 6.6; спуск перестановочен с пределами. Переходя к верхним целым частям и беря от них подвижные части, получаем требуемое неравенство

$$
\operatorname{Mov}\left\lceil m \mathbb{D}_{Y}+\mathbb{A}_{Y}\right\rceil \geqslant \operatorname{Mov}\left(f^{*} M+\left\lceil\mathbb{A}_{Y}-\varepsilon f^{*} G\right\rceil\right) \geqslant \operatorname{Mov} f^{*} M=f^{*} M
$$

Шаг 5: рациональность $\mathbb{D}$. Здесь, как и в предыдущем шаге, существенно используется размерность 2 .

\section{ЛЕмма 6.12. в-дивизор $\mathbb{D}$ рационален.}

ДоказАТЕльство. Напомним, что логпара $(X, B)$ предполагается терминальной. Следствие 6.6 утверждает, что все $\mathbb{D}_{i}$, а следовательно, также $\mathbb{D}$ спускаются на $X$. Так что для рациональности $\mathrm{b}$-дивизора $\mathbb{D}$ достаточно доказать рациональность его следа $\mathbb{D}_{X}$. Предположим противное, т.е. что $\mathbb{D}_{X}$ не рационален. Пусть $Y \rightarrow X-$ произвольное раздутие некоторого логразрешения пары $(X, B+G)$. Следствие 6.6 также утверждает, что асимптотическое $\mathbb{A}$-насьшение вьполнено (равномерно) на $Y$; в частности, при любом $i \gg 0$

$$
\operatorname{Mov}\left\lceil m \mathbb{D}_{Y}+\mathbb{A}_{Y}\right\rceil=\operatorname{Mov}\left\lceil m \mathbb{D}_{i Y}+\mathbb{A}_{Y}\right\rceil \leqslant m \mathbb{D}_{Y}
$$

Но по лемме 6.11 о необрашении в нуль имеем

$$
\operatorname{Mov}\left\lceil m \mathbb{D}_{Y}+\mathbb{A}_{Y}\right\rceil \geqslant(\bar{M})_{Y}=f^{*} M .
$$

Комбинируя с предыдущим неравенством насьшения, получаем

$$
m \mathbb{D}_{Y} \geqslant(\bar{M})_{Y}
$$

Следовательно, $m \mathbb{D}_{X}-M \geqslant 0$, что противоречит лемме 6.10 (iii).

Шаг 6: стабилизация. 
Лемма 6.13. Характеристическая последовательность $\mathbb{D}_{*}=\left\{\mathbb{D}_{i}\right\}$ стабилизируется, т.е. $\mathbb{D}=\mathbb{D}_{m}$ для некоторых $m \gg 0$.

ДОКАЗАТЕЛЬСТВО. Пусть $m \gg 0$ - некоторое целое число такое, что кратность $m \mathbb{D}_{X}$ свободна. Существование $m$ получаем из лемм 6.12 и 6.9 . По лемме о необращении в нуль с $M=m \mathbb{D}_{X}$

$$
\operatorname{Mov}\left\lceil m \mathbb{D}_{Y}+\mathbb{A}_{Y}\right\rceil \geqslant(\bar{M})_{Y}=f^{*} M=m \mathbb{D}_{Y} .
$$

Используя более точное насьщение, чем вьше,

$$
\operatorname{Mov}\left\lceil m \mathbb{D}_{Y}+\mathbb{A}_{Y}\right\rceil=\operatorname{Mov}\left\lceil m \mathbb{D}_{i Y}+\mathbb{A}_{Y}\right\rceil \leqslant m \mathbb{D}_{m Y}
$$

и неравенство $\mathbb{D}_{m} \leqslant \mathbb{D}$, получаем вместо противоречия соотношение

$$
\mathbb{D}_{m}=\bar{M} / m=\mathbb{D} .
$$

Тем самым доказана FGA-гипотеза 5.25 о конечной порожденности в размерности 2. Это немедленно вьводится из предельного критерия 5.21.

Значит, получен следуюший результат.

СлЕДСТвИЕ 6.14. 3-мерный klt логфлип, lt как в гипотезе 1.11 и даже lс логфлип существуют.

ДОКАЗАТЕЛЬСТвО. Непосредственно следует из теоремы 4.7, следствия 4.11, предложения 5.4, леммы 6.2 и теоремы 6.8. Доказательство существования lс логфлипа [36; 6.13] (см. определение в [36; Section 5]) требует 3-мерную логПММ и полуобильность в размерности 2 .

СлЕДСтвИЕ 6.15. 3-мерный направленный флоп и klt логфлоп существуют.

ДоказАТЕЛЬСТво. См. замечание 3.3 (ii).

\section{§ 7. 3-мерный полустабильный флип}

7.1. В предыдушем $\S 6$ было доказано существование 3-мерного klt логфлипа. Ясно, что из этого следует существование классического 3-мерного терминального (trm) флипа [26]: достаточно взять тривиальную границу $B=0$ и рассматривать многообразия только с терминальньми особенностями. Специальным случаем trm флипа является полустабильный 3-мерный флип, который возникает при изучении (относительной) ПММ для полустабильного вырождения, т.е. когда есть дополнительная структура - проективный (или собственный) морфизм $f: X \rightarrow \Delta$, где $\Delta=\operatorname{Spec} \mathscr{O}-$ спектр локального кольца $\mathscr{O}$. Здесь важно знать возникаюшие 3 -мерные особенности и согласовать 3 -мерные флипы (которые, как мы уже знаем, существуют и обрываются) с морфизмом $f$ и особенностями; в частности, показать, что флипы полустабильны (см. теорему 7.4 ниже). 
Возможно, первьй, кто изучал бирациональные преобразования - перестройки - в подобной ситуации, был Вик. С. Куликов [24]. В противоположность ПММ он использовал непроективные преобразования, даже если и предполагал исходную модель проективной. Хуже того, его перестройки обычно выводят из категории алгебраических многообразий. Основная цель этого параграфа - следствие 7.16 ниже, доказательство которого выявляет связь обеих моделей (Мори и Куликова; см. [35; Section 5], а также комментарий 7.17). Доказательство использует существование флипов (ср. с другим их применением в [38]) и классификацию возникаюших особенностей (см. [35] и замечание 7.6 ниже). Тем самым мы покажем, как из обших результатов типа существования флипа получить конкретное описание особенностей.

ОПРЕДЕЛЕнИЕ 7.2. Полустабильная особенность есть особенность относительной логпары $(X, S) \rightarrow \Delta=\operatorname{Spec~} \mathscr{O}$, где $f$ - собственный морфизм на спектр локального кольца $\mathscr{O}$, а $S$ - приведенньй дивизор таковы, что

(i) многообразие $X \mathbb{Q}$-факториально;

(ii) $(X, S)$ lt пара;

(iii) $S \sim 0 / \Delta$;

(iv) имеется полустабильное разрешение, т.е. логразрешение $g: Y \rightarrow X$ пары $(X, S)$, являющееся изоморфизмом вне $S$, с приведенным дивизором $g^{*} S$; разрешение предполагается лишь локально проективным над $\Delta$.

Отметим, что из (ii)-(iv) вытекает терминальность особенностей $X$. Действительно, в силу логтерминальности и (iv) это нужно проверить в изолированных точках на $S$, над которыми лежат исключительные b-дивизоры $E$ с $a(E, X, S)>-1$. Но $S$ - дивизор Картье по (iii). Откуда $a(E, X, 0)>0$ для таких b-дивизоров $E$ и точка $\mathrm{c}_{X} E$ терминальна. Отметим также, что по (iii) существует регулярная функция $t$ на $X$ над $\Delta$ с дивизором нулей $(t)=S$. ( $t$ единственна с точностью до умножения на обратимую функцию.) Особенности в настояшем параграфе обычно рассматриваются с точностью до аналитической (или формальной) эквивалентности. Основная схема $X$ предполагается 3 -мерной. Приведем типичные примеры.

Примеры 7.3. В примерах ниже морфизм $X \rightarrow \Delta$ тождественен. Пусть $A_{0}^{d}-$ локализация в точке $0=(0, \ldots, 0) d$-мерного аффинного пространства с координатами $x_{1}, \ldots, x_{d}$.

1. Тройная точка:

$$
X=\mathbb{A}_{0}^{3}, \quad S=\left(x_{1} x_{2} x_{3}\right) \text { и } t=x_{1} x_{2} x_{3},
$$

т.е. гранища $S=S_{1}+S_{2}+S_{3}$ имеет три простые компоненты $S_{i}=\left(x_{i}\right), i=1,2,3$, пересекаюшиеся в 0 . Точка 0 неособа (в лог-смысле).

2. Факторособенность. Для двух взаимно простых натуральных чисел $a \leqslant r$ положим

$$
X=\frac{1}{r}(a,-a, 1), \quad S=\left(x_{1} x_{2}\right) \subset X \quad \text { и } t=x_{1} x_{2},
$$

где $\frac{1}{r}(a,-a, 1)$ обозначает фактор схемы $\mathbb{A}_{0}^{3}$ по действию циклической групы $\mathbb{Z}_{r} \mathrm{c}$ весами $(a,-a, 1)$ : образуюшая $1 \in \mathbb{Z}_{r}$ действует по правилу

$$
\left(x_{1}, x_{2}, x_{3}\right) \mapsto\left(\zeta^{a} x_{1}, \zeta^{-a} x_{2}, \zeta x_{3}\right),
$$


где $\zeta=\sqrt[r]{1}$ - примитивный корень степени $r$ из 1 . Функция $t=x_{1} x_{2}$ инвариантна при данном действии и определена на $X$. При этом граница $S=S_{1}+S_{2}$ состоит из двух простых $\mathbb{Q}$-Картье поверхностей Вейля $S_{1}, S_{2}$, являюшихся факторами плоскостей $x_{1}=0$ и $x_{2}=0$ соответственно. Индекс точки 0 , т.е. индекс канонического дивизора $K$ в ней, совпадает с $r$. Данньй фактор полустабилен и особ в точности при $r>1$. Соответствуюшее разрешение, как и особенность, лучше всего описывать в торической категории - как последовательность взвешенных раздутий $[35 ; 1.2 .3],[32 ; 5.7]$ как в следующем примере.

3. Простейшая особенность типа $(r, a)$. Фактор гиперповерхностной особенности

$$
X=\left(x_{1} x_{2}=x_{3}^{r}+x_{4}^{n}\right) \subset \frac{1}{r}(a,-a, 1,0) \text { и } S=\left(x_{4}\right) \subset X,
$$

где $\frac{1}{r}(a,-a, 1,0)$ обозначает фактор схемы $\mathbb{A}_{0}^{4}$ по действию циклической групшы $\mathbb{Z}_{r}$ с весами $(a,-a, 1,0)$. Функция $t=x_{4}$ инвариантна при данном действии и определена на $X$. При этом граница $S$ является простым дивизором Картье. Индекс точки 0 совпадает с $r$. Данный фактор полустабилен и особ в точности при $r>1$. При $n=1$ получается особенность как в примере 2 , однако с другой границей $S=\left(x_{1} x_{2}-x_{3}^{r}=0\right)$. Полустабильное разрешение задается последовательностью взвешенных раздутий. Действительно, взвешенное раздутие с весами $\frac{1}{r}(a, r-a, 1, r)$ (см., например, [34; Appendix], [8; гл. II, 3.6]) имеет лишь полустабильные особенности и их не более трех:

а) одну простейшую, локально аналитически (или формально) изоморфную $X=$ $\left(x_{1} x_{2}=x_{3}^{r}+x_{4}^{n-1}\right) \subset \frac{1}{r}(a,-a, 1,0)$, в точности при $n>2$;

b) две факторособеннности, локально аналитически (или формально) изоморфные

$$
\frac{1}{a}(r \bmod a,-(r \bmod a), 1) \text { и } \frac{1}{r-a}(r \bmod r-a,-(r \bmod r-a), 1),
$$

где $x \bmod y$ обозначает остаток $0 \leqslant z<y$ при делении $x$ на $y$; это - особенности в точности при $a>1$ и $r-a>1$ соответственно.

Индукция по $n$ дает разрешение особенности в а). Аналогично индукцией по индексу $r$ разрешаются особенности в b). (См. подробности в [35; Section 4], [32; 5.7].)

ТЕОРема 7.4 (полустабильность флипа). 3-мерное экстремальное дивизориальное K-сдутие и флип полустабильны, т.е. сохраняют полустабильность особенностей.

Доказательство дается ниже вместе с доказательством предложения 7.9.

СлЕДСТвИЕ 7.5 (модель Мори). Любая 3-мерная проективная над $\Delta$ и допускающая лишь полустабильнье особенности схема имеет результирующую модель, особенности которой лишь полустабильны. При әтом относительная минимальная модель получается, если и только если кодаирова размерность слоя схемы $X$ над общей точкой в $\Delta$ будет $\geqslant 0$. 
ДоКАЗАТЕльСТво. Непосредственно следует из теоремы согласно ПММ и обрыву в теореме 3.5. При этом схемы можно заменять на многообразия над окрестностью точки с данной локализацией базы $\Delta$.

ЗАмЕчАниЕ 7.6. Само существование флипов в теореме 7.4 можно установить непосредственно. Исторически сначала было установлено существование именно таких флипов [41], [11], [35]. Накрытия позволяют установить существование более общих флипов [34; 2.6-9], и даже в положительной характеристике [15]. Более абстрактное доказательство предложено Корти [5] (см. также замечание 7.14 (ii) ниже). Его доказательство использует редукцию к допредельным, предельным и специальньм флипам из [34; Section 6] (см. также §4 вьше), которая сводит существование полустабильного флипа к ситуации специального полустабильного. Последнее легко доказывается с помошью сушествования 1-дополения в смысле второго автора (см. [34; Section 5], [21; Section 19]). До наших дней появляются конструкции полустабильных флипов и их обобщений [29]. Во всех этих конструкциях и доказательствах существенно используется классификация lс особенностей в размерности 2.

Утверждение теоремы 7.4 о дивизориальных сдутиях получается непосредственно из определения. Однако перестройки требуют более инвариантного (независящего от разрешения) подхода.

ОПреДЕлЕниЕ 7.7. Алгебраическое нормальное многообразие (или схема) называется аналитически (или формально) $\mathbb{Q}$-факториальныц $(\mathbb{Q}$-факториальной), если последнее вьполнено локально аналитически (соответственно формально) вблизи каждой точки, т.е. каждая точка аналитически (соответственно формально) $\mathbb{Q}$-факториальна.

Лемма 7.8. Всякое 3-мерное klt многообразие (пространство или схема) X и даже логпара имеет $\mathbb{Q}$-факториализачию (соответственно аналитическую или формальную $\mathbb{Q}$-факториализацию), т.е. существует (возможно непроективный) мальий собственный морфизм $Y \rightarrow X$ (соответственно аналитический, формальный над окрестностью компактного, полного подмножества в $X$ ) такой, что каждая точка $Y \mathbb{Q}$-факториальна (соответственно аналитически или формально $\mathbb{Q}$-факториальна).

ДокАЗАТЕЛЬСтво. Отметим, что в размерности 3 не $\mathbb{Q}$-факториальные точки во всех смыслах замкнуты и изолированы и их конечное число (для некоторой аналитической, формальной окрестности компактного, полного подмножества в $X$; в общем случае для всего аналитического пространства $X$ последнее неверно). Поэтому достаточно построить $\mathbb{Q}$-факториализацию над соответствующей окрестностью. Для конструции последней можно воспользоваться следствиями 6.7 и 6.14. (По замечанию 0.9 это применимо к аналитической и формальной категории.) Поскольку сдутие $Y \rightarrow X$ мало и 2-мерные klt особенности $\mathbb{Q}$-факториальны во всех смыслах, то $\mathbb{Q}$-факториальность в замкнутых точках кривых сдутия эквивалентна обычной $\mathbb{Q}$-факториальности окрестности кривых, т.е. локально над $X$ (в соответствуюшей топологии).

ПреДЛОЖенИЕ $7.9[35 ; 1.3,4.1]$. Росток любой точки 3-мерной относительной логпары $(X, S) \rightarrow \Delta$ с полустабильными особенностями аналитически (или 
формально) изоморфен одному из следующих:

(i) тройная точка примера 7.3.1;

(ii) факторособенность примера 7.3.2;

(iii) особенности типа $(r, a)$ : граница $S$ каждого такого ростка проста и его аналитическая (или формальная) $\mathbb{Q}$-факториализачия имеет лишь особенности, аналитически (или формально) изоморфные простейшим особенностямм типа $(r, a)$ примера 7.3 .3 (см. подробности в следующем комментарии).

КоммеНТАРИй $7.10[35 ; 1.3 .6,4.6]$. Тип особенности в (iii) теоремы определяется типом особенности поверхности $S$. Так, тип $(r, a)=(1,1)$ или, эквивалентно, индекс $r=1$ ровно тогда, когда особенность на $S$ дювалевская. При этом $\mathbb{Q}$-факториализация есть малое разрешение особенности и возможны все типы дювалевских особенностей.

Во всех остальных случаях в (iii), т.е. при индексе $r>1$, особенность на $S$ есть циклическая факторособенность типа $\frac{1}{r^{2}}(a, r-a)[14 ; 3.1]$. При этом исключительные кривые $\mathbb{Q}$-факториализации $Y \rightarrow X$ лежат на $S_{Y}-$ собственном прообразе $S$ на $\mathbb{Q}$-факториализации, являются $(-1)$-кривьми на минимальном разрешении поверхности $S_{Y}$ и составляют цепочку на $S_{Y}$. (См. подробности в доказательстве следствия 4.6 из [35].)

ОПРЕДЕЛЕНИЕ 7.11. Пара, многообразие или схема $X$ (аналитически или формально) стабильно $\mathbb{Q}$-факториальна вдоль дивизора Картье $S$, если любое конечное накрытие вдоль $S$ сохраняет (аналитическую или формальную) $\mathbb{Q}$-факториальность. Под конечньми накрытиями подразумеваются конечные морфизмы нормальных многообразий, индушированные алгебраической функцией $g(t)$, где $t=0$ - уравнение $S$, т.е. $S=(t)$, а индуцированность означает, что накрьвающее многообразие или схема есть нормализация расслоенного произведение $X \times_{\mathbb{A}^{1}} Z$ для морфизма $X \rightarrow \mathbb{A}^{1}$, $x \mapsto t(x)$, и конечного накрытия $Z \rightarrow \mathbb{A}^{1}$, отвечающего расширению $k\left(\mathbb{A}^{1}\right)(g)$ поля рациональных функций. Иначе говоря, накрытие является нормализацией $X$ в расширении $k(X)(g)$.

ПримеРЫ 7.12. 1. Пусть $(X, S)$ - аналитический росток логнеособой точки 0 3 -мерного многообразия с простой гранищей $S$, т.е. многообразие $X$ и непроводимая поверхность $S$ неособы в точке 0 . Тогда данная точка стабильно $\mathbb{Q}$-факториальна аналитически и формально. Достаточно рассмотреть формальные накрытия с одной ветвью, т.е. отвечающие алгебраической функции $g(t)=\sqrt[d]{t}$. Соответствующее накрытие логнеособо, а потому - $\mathbb{Q}$-факториально во всех смыслах.

2. Поместим теперь обыкновенную двойную точку в точке 0 на $S$, предполагая само многообразие $X$ неособым, т.е. оно формально эквивалентно $x^{2}+y^{2}+z^{2}+t=0$; при этом $S=(t)$. Функция $g=\sqrt{t}$ дает накрытие с особенностью, эквивалентной обыкновенной двойной $x^{2}+y^{2}+z^{2}+t^{2}=0$ и имеющей малое полустабильное разрешение. Значит, пара $(X, S)$ не стабильно $\mathbb{Q}$-факториальна. Пара $(X, S)$ также не полустабильна, поскольку не имеет полустабильного разрешения.

В том же стиле главньй технический результат параграфа, а именно следующая теорема. 
Теорема 7.13. Следующие утверждения о 3-мерном аналитическом (или формальном) ростке логпары $(X, S)$ в точке с простой границей $S$ әквивалентHbl:

(i) $(X, S)$ - простейшая особенность;

(ii) $(X, S)$ - полустабильная особенность, аналитически (или формально) $\mathbb{Q}$-факториальная, при этом полустабильное разрешение не обязательно предполагать проективным;

(iii) $(X, S)$ - стабильно $\mathbb{Q}$-факториальная, где $(X, S)$ - plt логпара и $S$ - дивизор Картье.

Особость точки в теореме не существенна по примеру 7.12.1.

ДОКАЗАТЕЛЬСТво тЕОРЕмЫ 7.13. (ii) следует из (i) по примеру 7.3.3. В частности, $\mathbb{Q}$-факториальность - по экстремальности взвешенных раздутий. (Более того, исключительные дивизоры двойственны кривым исключительного множества.)

(iii) следует из (ii) по следуюшему рассуждению из доказательства [5; 5.7]. Пусть $X^{\prime} \rightarrow X$ - конечное циклическое накрытие степени $d$ вдоль $S$, а $g: Y \rightarrow X$ - полустабильное разрешение. Рассмотрим расслоенное произведение $Y^{\prime}=Y \times_{X} X^{\prime}$. Пусть $D^{\prime} \in \operatorname{Div} X^{\prime}$ - дивизор Вейля. Следует показать, что $D^{\prime} \mathbb{Q}$-Картье.

У тверждение вытекает из следуюших замечаний.

a) Росток $Y^{\prime}$ нормален; в частности, естественное сдутие $g^{\prime}: Y^{\prime} \rightarrow X^{\prime}$ отвечает разложению Штейна морфизма $Y^{\prime} \rightarrow X$. Кроме того, $Y^{\prime}$ имеет тороидальные симлициальные, а потому аналитически (формально) $\mathbb{Q}$-факториальные особенности.

b) Циклическая группа $\mu_{d}$ действует на $Y^{\prime}$ и $X^{\prime}$ так, что $Y^{\prime} / \mu_{d}=Y$ и $X^{\prime} / \mu_{d}=X$.

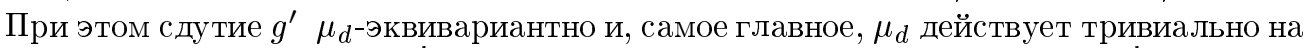
границе $Y^{\prime \mu_{d}}=S^{\prime}=\pi_{*}^{-1} S_{Y}^{\log }$, где $\pi: Y^{\prime} \rightarrow Y$ - морфизм факторизации, $S_{Y}^{\log }=S_{Y}+$ $\sum E_{i}$ - логобраз границы, а $E_{i}-g$-исключительные простые дивизоры, отождествляемые с $\pi_{*}^{-1} E_{i}$ на $Y^{\prime}$.

Действительно,

$$
\sum_{\zeta \in \mu_{d}} \zeta g_{*}^{\prime-1} D^{\prime}=\pi^{*} \pi_{*} g_{*}^{\prime-1} D^{\prime} .
$$

Более того, так как в силу аналитической $\mathbb{Q}$-факториальности $Y$ выполнена эквивалентность $\pi_{*} g_{*}^{\prime-1} D^{\prime}+\sum e_{i} E_{i} \sim_{\mathbb{Q}} 0$ над $X$ для некоторых $e_{i} \in \mathbb{Q}$, то

$$
\sum_{\zeta \in \mu_{d}} \zeta g_{*}^{\prime-1} D^{\prime}+\sum d e_{i} E_{i}=\pi^{*} \pi_{*}\left(g_{*}^{\prime-1} D^{\prime}+\sum e_{i} E_{i}\right) \sim_{\mathbb{Q}} 0
$$

над $X^{\prime}$. Отсюда

$$
g_{*}^{\prime-1} D^{\prime}+\sum e_{i} E_{i} \equiv 0
$$

над $X^{\prime}$, поскольку по а) пересечение с любой кривой $C \subset S^{\prime}$ над $X^{\prime}$ определено, а по b) $\zeta g_{*}^{\prime-1} D^{\prime} C=\zeta g_{*}^{\prime-1} D^{\prime} \zeta C=g_{*}^{\prime-1} D^{\prime} C$. Росток $X^{\prime}$ имеет лиш рациональные особенности как $X$. Значит, численную эквивалентность $\equiv$ над $X^{\prime}$ можно заменить на $\sim_{\mathbb{Q}}$, т.е. $D^{\prime} \mathbb{Q}$-Картье. 
(i) следует из (iii) по существу из теоремы о полустабильной редукции. Согласно предположениям, особенность является гиперповерхностной факторособенностью типа

$$
\left(x_{1} x_{2}=F\left(x_{3}^{r}, x_{4}=t\right)\right) \subset \frac{1}{r}(a,-a, 1,0)
$$

с $F\left(x_{3}^{r}, 0\right) \neq 0$. По стабильной $\mathbb{Q}$-факториальности после аналитической или формальной замены координат $F\left(x_{3}^{r}, x_{4}\right)=x_{3}^{r}+x_{4}^{n}$ (cp. с [14], где и были введены простейшие (moderate по английски) особенности).

ЗАмЕчАнИя 7.14. (i) Смысл простейших особенностей в их дискретности: каждая из них кроме типа $(r, a)$ имеет еше один натуральньй параметр $n$. При этом общая деформация простейшей точки типа $(r, a)$ отвечает гиперповерхности $x_{1} x_{2}=x_{3}^{r}+x_{4}$ с $n=1$, а потому является факторособенностью.

(ii) Стабильная $\mathbb{Q}$-ракториальность для описания простейших особенностей - одно из новшеств Корти в [5], где также дано обобщение теоремы 7.4 на более широкий класс особенностей (ср. с его несколько вольньм определением полустабильности особенности $[5 ; 2.5]$; в отечественной терминологии такие особенности назьвают q-полустабильными [29]).

(iii) Вероятно, один из возможных ответов на вопрос Корти в начале [5; Section 5] - многомерное обобщение теоремы 7.13 об эквивалентности аналитической (формальной) стабильной и обычной $\mathbb{Q}$ - факториальности особенностей, имеющих полустабильное разрешение. Однако их явное описание следует ожидать лишш для достаточно мягких, например, когда дискрепантности над точкой $>d-3$ в размерности $d$.

ДОКАЗАТЕЛЬСТВО ТЕОРЕМЫ 7.4 И ПРЕДЛОЖЕНИЯ 7.9. Как бЫЛо отмечено выше, для доказательства теоремы 7.4 достаточно проверить полустабильность экстремального флипа. Иначе говоря, надо проверить, что полустабильность особенностей сохранится при флипе. Отметим, что сохранение свойств (i)-(iii) полустабильных особенностей вытекает из обших свойств экстремальных логфлипов. Трудность возникает со свойством (iv) о сушествовании полустабильного разрешения. Однако это свойство прямо следует из теоремы 7.13 в случае аналитически (или формально) $\mathbb{Q}$-факториальных особенностей. Действительно, экстремальные флипы сохраняют аналитическую (формальную) $\mathbb{Q}$-факториальность и ее стабильную версию, в частности, простейшие особенности. Общие полустабильные флипы сводятся к таким с помощью аналитической (или формальной) $\mathbb{Q}$-факторализации (см. лемму 7.8). При этом хорошо известные особенности предложения 7.9 (i), (ii) аналитически (формально) стабильно $\mathbb{Q}$-факториальны.

Конечно, $\mathbb{Q}$-факториализация в свою очередь нуждается в полустабильности фллипов, чтобы приводить к полустабильным особенностям. Поэтому для завершения доказательства теоремы и предложения 7.9 (iii) надо использовать индукцию по минимальному числу исключительных простых дивизоров, необходимых в полустабильном разрешении. При этом в каждом индукционном шаге сначала устанавливается предложение 7.9 (iii) и лиш затем теорема.

A posteriori допустима обычная алгебраическая проективность над $X$ (и над $\Delta$ ) полустабильного разрешения (как в определении 7.2 (iv)), когда $X$ - алгебраическое (проективное над $\Delta$ ) многообразие или схема $[35 ; 4.11]$, а потому такое разрешение и само алгебраично. 
ЗАмечАниЕ 7.15. (Ср. с замечанием 7.14 (ii).) Смысл полустабильности особенностей в их обшности (см. подробнее $[35 ; 4.7])$. Согласно предложению 7.9 (iii) и замечанию 7.14 (i), общая деформация при фиксированной особенности на $S$ полустабильной точки типа $(r, a)$ имеет аналитическую (формальную) $\mathbb{Q}$-факториализацию лишь с тем же числом исключительных кривых и факторособенностями того же типа; в частности, при индексе $r=1$ такая $\mathbb{Q}$-факториализация дает малое разрешение.

Вероятно, это может дать простое объяснение, почему факторособенность в гипотезе Маккея имеет малое разрешение, и привести к обобщениям данного результата. Действительно, факторособенности должны быть недеформируемыми при фиксированной особенности на $S$ и обшими. Хотя утверждение, по-видимому, справедливо, оно требует объяснения. Тогда малое разрешение получается как аналитическая (формальная) $\mathbb{Q}$-факториализация.

Возможное обобщение в размерности 3: аналитическая (формальная) $\mathbb{Q}$-факториализация терминальной факторособенности индекса $r$ имеет лишь факторособенности того же индекса или их индекс делит $r$. Для многомерных обобщений терминальности, скорее всего, недостаточно, а надо требовать достаточную мягкость особенностей, например, как в замечании 7.14 (iii).

СлЕдСтвИЕ 7.16 (модель Куликова). Любая 3-мерная проективная над $\Delta u$ допускающая лишь полустабильные особенности схема имеет результирующую аналитическую (формальную) модель, особенности которой лишь полустабильные особенности примеров 7.3.1-3 с $r \geqslant 2$ в примере 7.3.3. При этом относительная минимальная модель получается, если и только если кодаирова размерность слоя схемь $X$ над общей точкой в $\Delta$ будет $\geqslant 0$.

Более того, результирующая модель имеет тройные точки из примера 7.3.1 (соответственно особые двойнье из примера 7.3.2), только если их имеет исходная схема (соответственно двойныее или тройные).

ДОКАЗАТЕЛЬСТВО. В силу предложения 7.9 (iіi) получается аналитической (или формальной) $\mathbb{Q}$-факториализацией из модели следствия 7.5. При $r=1$ это дает малое логразрешение.

Утверждение о тройных (особых двойных) точках требует связности логканонических особенностей $[34 ; 5.7]$.

КомментАРИй 7.17. Доказательство следствия 7.16 показывает, что модель Куликова строится по модели Мори. Однако, вероятно, модель Куликова и в более общей ситуации, а не только для полустабильного вырождения К3 поверхностей можно построить непосредственно, работая в непроективной категории. Главная трудность при этом - в конструкции экстремальных сдутий аналитически (формально), особенно, малых. Тем самым, проективность при определении полустабильных особенностей, даже локальная в аналитическом смысле, возможно, не нужна вообще.

С другой стороны, работая в алгебраической категории Мори, т.е. в проективной ПММ, мы сохраним полустабильность (см. теорему сравнения [ $35 ; 4.11]$ и последуюшие результаты в [35; Section 4]). То же самое, вероятно, относится к алгебраической непроективной категории и к алгебраическим пространствам.

СледСТвИЕ 7.18. Пусть $f: X \rightarrow \Delta$ - проективное полустабильное вырождение неособых поверхностей с численно тривиальным каноническим дивизором, 
m.е. $\Delta=\operatorname{Spec~} \mathscr{O}$ - спектр (локального) кольца дискретного нормирования $\mathscr{O}$, логпара $(X, B)$ с границей $B=f^{-1} 0-$ чентральный слой - полустабильная логнеособая. При этом поверхность $X_{\eta}$ для общей точки $\eta \in \Delta$ имеет численно тривиальный дивизор $K_{X_{\eta}}$ или, әквивалентно, $m K_{X_{\eta}} \sim 0$ для некоторого натурального $m>0$. Тогда имеется его полустабильная аналитическая (формальная) модель над $\Delta$, являющаяся полустабильной минимальной и особенности которой - лишь полустабильные особенности примеров 7.3.1-3 с $r \geqslant 2$ в примере 7.3.3. Кроме того, $r$ делит $m$.

Более того, результирующая модель имеет тройнье точки из примера 7.3.1 (соответственно особые двойные из примера 7.3.2), только если их имеет исходная модель (соответственно двойные или тройные).

ДоКАЗАТЕЛЬСТво. Непосредственно по следствию 7.16. Отметим также, что индекс горенштейновости на минимальной модели делит $m$ по схемной приведенности $f^{*} 0=f^{-1} 0$ центрального слоя, откуда $r$ делит $m$.

ЗАмЕЧАНИЕ 7.19. По классификации поверхностей в следствии 7.18 можно полагать $m=1,2,3,4$ или 6 . Точнее, $m=1$ за исключением поверхностей Энриквеса с $m=2$ и гиперэллиптических поверхностей с $m=2,3,4$ или 6.

ПримеРЫ 7.20. 1. (Полустабильные вырождения К3 поверностей.) Если $X_{\eta}-$ К3-поверхность, то $m=1$ и минимальная модель в следствии 7.18 логнеособа, но, возможно, непроективна. Эта - главный результат работы Куликова [24]. По конструкции данная модель есть аналитическая (формальная) $\mathbb{Q}$-факториализация проективной модели Мори следствия 7.5.

2. (Полустабильные вырождения поверхностей Энриквеса.) Для таких вырождений $m=2$. Поэтому это особенности как в примерах 7.3.2 и 7.3.3 с $r=2, a=1$. То же относится к вырождению гиперэллиптических поверхностей с $m=2$. При этом если исходная модель не имеет тройных точек, то модель Куликова будет иметь лишш особенности как в примере 7.3.3 с $r=2, a=1$, имеющие разрешение в форме цветочного горшка $[28 ; 3.3 .1]$. Вырождения с такими особенностями действительно сушествуют, что указьвает на ошибку в методах Куликова для поверхностей Энриквеса [24]; по-видимому, в силу численности этих методов.

\section{СПИСОК ЛИТЕРАТУРЫ}

[1] F. Ambro. Quasi-log varieties // Труды МИАН. 2003. Т. 240. С. 220-239.

[2] V. V. Batyrev. The cone of effective divisors on threefolds // Contemp. Math. 1992. V. 131. Part 3. P. 337-352.

[3] А. А. Борисов, В. В. Шокуров. Направленные рациональные приближения с некоторыми приложениями в алгебраической геометрии // Труды МИАН. 2003. Т. 240. С. 73-81.

[4] И.А. Чельцов. О гладкой четырехмерной квинтике // Матем. сб. 2000. Т. 191. № 9. C. $139-162$.

[5] A. Corti. Semistable 3-fold flips // alg-geom/9505035.

[6] A. Corti. Factoring birational maps of threefolds after Sarkisov // J. Algebraic Geom. 1995. V. 4. № 2. P. 223-254.

[7] A. Corti. 3-fold flips after Shokurov // Preprint. Chicago: Univ. of Chicago, 2003.

[8] В.А. Исковских. Бирациональная жесткость гиперповерхностей Фано в рамках теории Мори // УМН. 2001. Т. 56. №2. С. 3-86. 
[9] В.А. Исковских. b-Дивизоры и функциональные алгебры по Шокурову // Труды МИАН. 2003. Т. 240. С. 8-20.

[10] В. А. Исковских. О работе Шокурова "Prelimiting flips" // Труды МИАН. 2003. Т. 240. C. 21-42.

[11] Y. Kawamata. Crepant blowing-up of 3-dimensional canonical singularities and its application to degenerations of surfaces // Ann. of Math. (2). 1988. V. 127. №1. P. 93-163.

[12] Y. Kawamata. Termination of log flips for algebraic 3-folds // Internat. J. Math. 1992. V. 3. № 5. P. 653-659.

[13] Y. Kawamata. Abundance theorem for minimal threefolds // Invent. Math. 1992. V. 108. № 2. P. 229-246.

[14] Y. Kawamata. Moderate degenerations of algebraic surfaces // Complex Algebraic Varieties (Bayreuth, 1990) / ed. K. Hulek et al. Berlin: Springer-Verlag, 1992. P. 113-132. (Lecture Notes in Math. V. 1507.)

[15] Y. Kawamata. Semistable minimal models of threefolds in positive or mixed characteristic // J. Algebraic Geom. 1994. V. 3. № 3. P. 463-491.

[16] Y. Kawamata. On the cone of divisors of Calabi-Yao fiber spaces // Internat. J. Math. 1997. V. 8. № 5. P. 665-687.

[17] Y. Kawamata, K. Matsuda, K. Matsuki. Introduction to the minimal model problem // Algebraic Geometry (Sendai, 1985) / ed. T. Oda. Amsterdam: North-Holland, 1987. P. 283-360. (Adv. Stud. Pure Math. V. 10.)

[18] Y. Kawamata, K. Matsuki. The number of the minimal models for a 3-fold of general type is finite // Math. Ann. 1987. V. 276. № 4. P. 595-598.

[19] S. Keel, K. Matsuki, J. McKernan. Log abundance theorem for threefolds // Duke Math. J. 1994. V. 75. № 1. P. 99-119; corrections: ibid. 2004. V. 122. № 3. P. 625-630.

[20] J. Kollár. Flops // Nagoya Math. J. 1989. V. 113. P. 15-36.

[21] J. Kollár (ed.). Flips and Abundance for Algebraic Threefolds. A Summer Seminar at the University of Utah (Salt Lake City, 1991). Paris: Soc. Math. France, 1992. (Astérisque. № 211.)

[22] J. Kollár, S. Mori, with the collaboration of C. H. Clemens, A. Corti. Birational Geometry of Algebraic Varieties. Cambridge: Cambridge Univ. Press, 1998. (Cambridge Tracts in Math. V. 134.)

[23] J. Kollár, N. I. Shepherd-Barron. Threefolds and deformations of surface singularities // Invent. Math. 1988. V. 91. № 2. P. 299-338.

[24] В.С. Куликов. Вырождения К3 поверхностей и поверхностей Энриквеса // Изв. АН СССР. Сер. матем. 1977. Т. 41. № 5. С. 1008-1042.

[25] K. Matsuki. Introduction to the Mori Program. New York: Springer-Verlag, 2002.

[26] S. Mori. Flip theorem and the existence of minimal models for 3-folds // J. Amer. Math. Soc. 1988. V. 1. № 1. P. 117-253.

[27] N. Nakayama. The lower semicontinuity of the plurigenera of complex varieties // Internat. J. Math. 1997. V. 8. № 5. P. 551-590.

[28] U. Persson. On degenerations of algebraic surfaces // Mem. Amer. Math. Soc. 1977. V. 189.

[29] Ю. Г. Прохоров. О полустабильных стягиваниях Мори // Изв. РАН. Сер. матем. 2004. T. 68 . № 2. C. $147-158$.

[30] M. Reid. Canonical 3-folds // Journeés de Géométrie Algébrique d'Angers (Angers, 1979) / ed. A. Beauville. Alphen: Sijthoff and Noordhoff, 1980. P. 273-310.

[31] M. Reid. Minimal models of canonical 3-folds // Algebraic Varieties and Analytic Varieties (Tokyo, 1981). Amsterdam: North-Holland, 1983. P. 131-180. (Adv. Stud. Pure Math. V. 1.)

[32] M. Reid. Young person's guide to canonical singularities // Proc. Sympos. Pure Math. 1987. V. 46. № 1. P. 345-414.

[33] В. В. Шокуров. Теорема о необращении в нуль // Изв. АН СССР. Сер. матем. 1985. T. 49. № 3. C. $635-651$.

[34] В.В. Шокуров. Трехмерные логперестройки // Изв. АН СССР. Сер. матем. 1992. T. 56. № 1. C. 105-203. 
[35] V. V. Shokurov. Semistable 3-fold flips // Изв. АН СССР. Сер. матем. 1993. Т. 57. № 2. C. $165-222$.

[36] V. V. Shokurov. 3-fold log models // J. Math. Sci. 1996. V. 81. №3. P. 2667-2699.

[37] V. V. Shokurov. Prelimiting flips // Труды МИАН. 2003. Т. 240. C. 82-219.

[38] V. V. Shokurov. Letters of a bi-rationalist. IV. Geometry of log flips // Algebraic Geometry / ed. M.C. Beltrametti et al. Berlin: de Gruyter, 2002. P. 313-328.

[39] В.В. Шокуров. Письма о бирациональном. V: М.л.д. и обрыв логфилипов // Труды МИАН. 2004. Т. 246. С. 328-351.

[40] H. Takagi. 3-fold log flips according to V.V. Shokurov // Preprint, 1999.

[41] S. Tsunoda. Degenerations of surfaces // Internat. J. Math. 1997. V. 8. № 5. P. 755-764.

Математический институт им. В. А. Стеклова РАН;

Поступила в редакцию Johns Hopkins University, Baltimore, USA

15.10 .2004

E-mail: shokurov@math.jhu.edu, shokurov@mi.ras.ru;

iskovsk@mi . ras.ru 